\title{
HUMAN FACTORS ENGINEERING IN SONAR VISUAL DISPLAYS
}

by

\author{
LAWRENCE FRANCIS GALVIN
}

B. S. Mathematics, United States Naval Academy

(1978)

Submitted in Partial Fulfillment of the

Requirements for the Degree of

MASTER OF SCIENCE in OCEANOGRAPHIC ENGINEERING

at the

MASSACHUSETTS INSTITUTE OF TECHNOLOGY

and the

WOODS HOLE OCEANOGRAPHIC INSTITUTION

August 1991

(c) Lawrence F. Galvin, 1991

The author hereby grants to MIT, WHOI, and the United States Government permission to reproduce and to distribute copies of this thesis document in whole or in part.

Signature of Author

Joint Program in Oceanographic Engineering

Massachusetts Institute of Technology

Woods Hole Oceanographic Institution
August 1, 1991 ARINEE

Certified by

Dr. W. Kenneth Stewart

Woods Hole Oceanographic Institution RARY

Thesis Supervisor

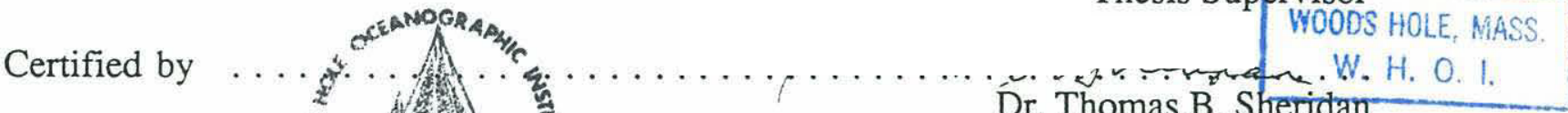

Massachusetts Institute of Technology

Thesis Sunervisnr

Accepted by $\ldots \ldots \ldots \ldots \ldots \ldots \ldots$

Chairman, Joint Committee for Oceanographic Engineering, Massachusetts Institute of Technology / Woods Hole Oceanographic Institution 


\title{
HUMAN FACTORS ENGINEERING IN SONAR VISUAL DISPLAYS
}

\author{
by
}

\author{
LAWRENCE FRANCIS GALVIN
}

\author{
Submitted to the Massachusetts Institute of Technology/ \\ Woods Hole Oceanographic Institution \\ Joint Program in Oceanographic Engineering \\ on August 30,1991, in partial fulfillment of the \\ requirements for the degree of
}

\section{MASTER OF SCIENCE IN OCEANOGRAPHIC ENGINEERING}

\begin{abstract}
Undersea technology is on the verge of equipping remotely operated vehicle (ROV) pilots with a three-dimensional (3-D), real-time display incorporating data from a wide variety of sensors including sonar (sound navigation and ranging), cameras, and lasers. Effective collection, computation, and presentation of this data to the pilot in a single display presents hardware, software and human factors problems. This thesis focuses on human factors issues associated with the display of information which could enhance the pilot's efficiency of performance. Background information on human factors engineering, 3-D computer graphics displays, and application of the 3-D perspective display precede the details of the experiment.
\end{abstract}

Five specific display enhancements tested include altering the displayed field of view, providing a screen grid, displaying the current range to the target of interest, using a vertical color scheme, and controlling the display update rate. Seven tests measure the effects of these display enhancements on the simulated piloting of an ROV. The effects of the ROV simulation and operator learning curves are removed to compare performance changes due to the various enhancements directly. Operator comments during and after testing as well as test monitor/author observations provide insight into the experiment. Test result implications for system design trade-offs are discussed in detail. Recommendations for future research and the proposed construction of a fully equipped ROV simulator complete the work.

Thesis Supervisors:

Dr. W. Kenneth Stewart

Assistant Scientist, Deep Submergence Laboratory

Woods Hole Oceanographic Institution

Dr. Thomas B. Sheridan

Professor of Engineering and Applied Psychology

Massachusetts Institute of Technology 


\section{Acknowledgements}

Initially, I wish to thank the United States Navy for this unsurpassed opportunity to improve myself professionally.

A number of individuals contributed directly to the completion of this work. Key computer programming assistance was provided by Charlie Chafouleas and Dan Potter. Tagore Somers and Martin Bowen of the Marquest Group, Inc., lent equipment. Will Sellers and Skip Gleason injected their ROV pilot experience. Rob Keefe, Hanu Singh, Gary Edwards, and Tom Riggle each spent many hours flying the simulator. Special thanks is due to CDR John Halsema, USN, Dr. Dana Yoerger, and Dr. Gene Terray.

Sincere gratitude is due to Raymond A. Sullivan, M.D. and Thomas F. Gregg, M.D., for services rendered.

I have been fortunate to have two extremely knowledgeable, capable, and respected thesis advisors in Dr. Ken Stewart (WHOI) and Dr. Tom Sheridan (MIT). Perhaps the greatest professional contribution to my work was provided by Dr. Stewart, who for the entire two years of my stay has understood even better than I the rigors of raising a family while attending graduate school.

Without doubt, the greatest personal contribution to my work came from my wife Atti. In our short tour here she has given birth to two healthy baby boys (Danny - June 15, 1989 and Timmy - March 27, 1991) while continuing to run the house and our oldest son Andy (September 14, 1987). She is living proof of the saying: "Navy Wife - It's the toughest job in the Navy."

Finally, I must offer my appreciation to people of the Woods Hole Oceanographic Institution. Top to bottom it is the singularly finest working environment it has been my great pleasure to experience. 


\section{Dedication}

To my most special wife Atti

and

our wonderful sons Andy, Danny, and Timmy. 


\section{Table of Contents}

Page

$\begin{array}{ll}\text { Abstract } & 2\end{array}$

Acknowledgements 3

Dedication $\quad 4$

Table of Contents $\quad 5$

List of Figures $\quad 8$

$\begin{array}{ll}\text { List of Tables } & 10\end{array}$

$\begin{array}{ll}\text { Chapter 1. Introduction } & 13\end{array}$

$\begin{array}{lll}1.1 \text { Background } & 13\end{array}$

$\begin{array}{ll}1.2 \text { Research Objectives } & 14\end{array}$

$\begin{array}{ll}1.3 \text { Terminology } & 15\end{array}$

$\begin{array}{ll}1.4 \text { Overview } & 16\end{array}$

$\begin{array}{ll}\text { Chapter 2. Human Factors Engineering } & 17\end{array}$

$\begin{array}{ll}2.1 \text { Objectives and Doctrines } & 17\end{array}$

$\begin{array}{ll}2.2 \text { Methodology Issues } & 17\end{array}$

$\begin{array}{lr}2.3 \text { Research Criteria } & 18\end{array}$

$\begin{array}{lr}2.4 \text { Visual-Coding } & 19\end{array}$

$\begin{array}{ll}2.5 \text { Control and Tracking Performance } & 19\end{array}$

$\begin{array}{ll}2.6 \text { Test and Evaluation } & 22\end{array}$

Chapter 3. Three-Dimensional Computer Graphics Displays 23 
3.1 True 3-D Displays 23

3.2 Simulated 3-D Displays 24

$\begin{array}{ll}3.3 \text { The Marquest System } & 25\end{array}$

3.4 The Display and the Monitor 26

Chapter 4. Application of the 3-D Perspective Display 29

4.1 Ocean Environmental Sensing 29

4.2 Multisensor Modeling 30

4.3 The Deep Submergence Laboratory System 31

4.4 Targeted Human Factors 33

Chapter 5. Testing Display Effectiveness 35

5.1 Equipment 35

5.2 Data Point Courses 35

5.3 Modeling the Jason Vehicle 38

5.4 Operators and Training 41

5.5 Test 1 - Field of View 42

5.6 Tests 2 and 3 - Gridding 42

5.7 Tests 4 and 5 - Vertical Color Schemes 43

5.8 Test 6 - Heads Up Range 45

5.9 Test 7 - Display Update Rate 45

Chapter 6. Testing Results $\quad 47$

$\begin{array}{ll}6.1 \text { General } & 47\end{array}$

6.2 Operator Learning Curves $\quad 47$

6.3 Field of View $\quad 52$

6.4 Gridding $\quad 52$ 
6.5 Vertical Color Schemes $\quad 52$

6.6 Heads-Up Range $\quad 54$

6.7 Display Update Rate 54

6.8 Operator Comments 55

6.9 Test-Monitor Observations $\quad 57$

Chapter 7. Summary, Conclusions and Recommendations 60

$\begin{array}{ll}7.1 \text { Summary } & 60\end{array}$

$\begin{array}{ll}7.2 \text { Conclusions } & 60\end{array}$

$\begin{array}{ll}7.3 \text { Recommendations } & 62\end{array}$

$\begin{array}{ll}\text { Appendix A. Nomenclature } & 64\end{array}$

Appendix B. Abbreviations 66

$\begin{array}{ll}\text { Appendix C. Target and Grid Design } & 68\end{array}$

Appendix D. Data Point Courses 71

$\begin{array}{ll}\text { Appendix E. Raw Data } & 103\end{array}$

$\begin{array}{ll}\text { Appendix F. Normalized Data } & 116\end{array}$

$\begin{array}{ll}\text { Appendix G. Operator Learning Curves } & 129\end{array}$

Appendix H. References 133

$\begin{array}{ll}\text { Biographical Note } & 136\end{array}$ 


\section{List of Figures}

Title

Page

2.1 The RGB (red, green, and blue) cube

20

3.1 Monitor screen during test 6

4.1 DSL comparison of underwater remote-sensing systems

5.1 Visualization of data point course constraints 39

5.2 The world coordinate system 40

5.3 The vehicle coordinate system 40

6.1 Normalized mean times $\left(60^{\circ} \mathrm{FOV}\right)$

6.2 Learning-corrected, normalized mean times $\left(60^{\circ} \mathrm{FOV}\right)$

6.3 Learning-corrected, normalized mean times (varying FOV) 53

C.1 2-D grid (operator's display with $60^{\circ} \mathrm{FOV}$ and target course $\mathrm{X}$ ) 70

C.2 3-D grid (operator's display with $60^{\circ}$ FOV and target course X) 70

D.1 Course A (operator's display with $\left.60^{\circ} \mathrm{FOV}\right)$

D.2 Course B (operator's display with $60^{\circ} \mathrm{FOV}$ ) 75

D.3 Course C (operator's display with $60^{\circ} \mathrm{FOV}$ ) 78

D.4 Course D (operator's display with $60^{\circ} \mathrm{FOV}$ ) 78

D.5 Course E (operator's display with $\left.60^{\circ} \mathrm{FOV}\right)$ 
D.6 Course F (operator's display with $60^{\circ} \mathrm{FOV}$ ) 81

D.7 Course G (operator's display with $60^{\circ} \mathrm{FOV}$ ) 84

D.8 Course H (operator's display with $60^{\circ} \mathrm{FOV}$ ) 84

D.9 Course I (operator's display with $60^{\circ} \mathrm{FOV}$ ) 87

D.10 Course J (operator's display with $60^{\circ} \mathrm{FOV}$ ) 87

D.11 Course K (operator's display with $60^{\circ} \mathrm{FOV}$ ) 90

D.12 Course L (operator's display with $60^{\circ} \mathrm{FOV}$ ) 90

D.13 Course M (operator's display with $60^{\circ} \mathrm{FOV}$ ) 93

D.14 Course N (operator's display with $60^{\circ}$ FOV) 93

D.15 Course O (operator's display with $60^{\circ} \mathrm{FOV}$ ) 96

D.16 Course P (operator's display with $60^{\circ} \mathrm{FOV}$ ) 96

D.17 Course Q (operator's display with $60^{\circ} \mathrm{FOV}$ ) 99

D.18 Course R (operator's display with $60^{\circ} \mathrm{FOV}$ ) 99

D.19 Course X (operator's display with $60^{\circ}$ FOV) 101

D.20 Course X (operator's display with $30^{\circ}$ FOV) 101

D.21 Course X (operator's display with $15^{\circ} \mathrm{FOV}$ ) 102

G.1 Operator I $60^{\circ}$ FOV learning curve 131

G.2 Operator II $60^{\circ}$ FOV learning curve 131

G.3 Operator III $60^{\circ}$ FOV learning curve 132

G.4 Operator IV $60^{\circ}$ FOV learning curve 132 


\section{List of Tables}

Title

Page

2.1 Color set RGB to HSB conversion

20

5.1 Data point course relationships 39

5.2 Dynamic vehicle simulation parameters 39

5.3 The color sets $(1,6,21)$ and associated altitude bands 44

6.1 Summed standard deviations (SSD), extracted values (EV), and $\begin{array}{ll}\text { standardization factors (SF) } & 50\end{array}$

6.2 Learning-corrected, normalized mean times ( $\left.60^{\circ} \mathrm{FOV}\right) \quad 50$

6.3 Learning-corrected, normalized mean times (varying FOV) 53

$\begin{array}{lll}\text { D.1 Course A } & 73\end{array}$

$\begin{array}{lll}\text { D.2 Course B } & 74\end{array}$

$\begin{array}{lll}\text { D.3 Course C } & 76\end{array}$

$\begin{array}{lll}\text { D. } 4 \text { Course D } & 77\end{array}$

$\begin{array}{lll}\text { D.5 Course E } & 79\end{array}$

$\begin{array}{lll}\text { D.6 } & \text { Course F } & 80\end{array}$

D.7 Course G 82

$\begin{array}{lll}\text { D. } 8 & \text { Course } \mathrm{H} & 83\end{array}$

$\begin{array}{lll}\text { D.9 Course I } & 85\end{array}$

D.10 Course J 86

$\begin{array}{lll}\text { D.11 Course K } & 88\end{array}$ 
$\begin{array}{llr}\text { D.12 Course L } & 89\end{array}$

$\begin{array}{lll}\text { D.13 Course M } & 91\end{array}$

D.14 Course N 92

$\begin{array}{lll}\text { D.15 Course O } & 94\end{array}$

$\begin{array}{lll}\text { D.16 Course P } & 95\end{array}$

D.17 Course Q 97

$\begin{array}{lll}\text { D. } 18 & \text { Course R } & 98\end{array}$

$\begin{array}{lll}\text { D.19 Course X } & 100\end{array}$

$\begin{array}{lll}\text { E.1 Operator I - Test } 1 & 104\end{array}$

$\begin{array}{ll}\text { E.2 Operator I - Tests 2, 3, and } 4 & 105\end{array}$

$\begin{array}{lll}\text { E.3 Operator I - Tests 5, 6, and 7 } & 106\end{array}$

E.4 Operator II - Test 1

$\begin{array}{lll}\text { E.5 Operator II - Tests 2, 3, and } 4 & 108\end{array}$

E.6 Operator II - Tests 5, 6, and 7 109

$\begin{array}{lll}\text { E.7 Operator III - Test } 1 & 110\end{array}$

E.8 Operator III - Tests 2, 3, and $4 \quad 111$

E.9 Operator III - Tests 5, 6, and 7 112

$\begin{array}{lll}\text { E.10 Operator IV - Test } 1 & 113\end{array}$

E.11 Operator IV - Tests 2, 3, and 4 114

E.12 Operator IV - Tests 5, 6, and 7 115

$\begin{array}{lll}\text { F.1 Operator I - Test } 1 & 117\end{array}$

$\begin{array}{ll}\text { F.2 Operator I - Tests 2, 3, and } 4 & 118\end{array}$

$\begin{array}{lll}\text { F.3 Operator I - Tests 5, 6, and 7 } & 119\end{array}$

$\begin{array}{lll}\text { F.4 Operator II - Test } 1 & 120\end{array}$

F.5 Operator II - Tests 2, 3, and 4 
F.6 Operator II - Tests 5, 6, and 7

F.7 Operator III - Test 1

F. 8 Operator III - Tests 2, 3, and 4

F.9 Operator III - Tests 5, 6, and 7

F.10 Operator IV - Test 1

F.11 Operator IV - Tests 2, 3, and 4

F.12 Operator IV - Tests 5, 6, and 7 


\section{Chapter 1}

\section{Introduction}

\subsection{Background}

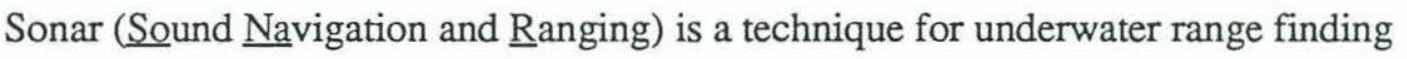
that has existed for more than fifty years. The author's thirteen years of experience with military and commercial sonars indicate that the techniques employed to present sonar information to the user significantly lag the technology used in areas such as hardware design, beam forming, and data processing, and unnecessarily limit the bandwidth of information transfer. The wide variety of display designs and information formats proves confusing to all but the most adept and experienced users. Additionally, the typical restriction of the presentation to two coding dimensions, coincident with the physical dimensions of the cathode ray tube (CRT), severely restricts the ability to effectively convey large amounts of data.

The Woods Hole Oceanographic Institution's (WHOI) Deep Submergence Laboratory (DSL) operates several seagoing craft including remotely operated vehicles (ROVs), whose pilot "flies" the deep-diving ROV from an operations center located on a mother ship on the surface above. Within video camera range the pilot's display resembles the view a driver sees out the front windshield of an automobile, with some loss of depth perception. This video display format is "natural" for the pilot and provides a large information transfer bandwidth. Suspended sediment near the bottom frequently limits the already restricted visual range of camera equipment to a couple of meters. Beyond visual range the pilot 
must rely on the customary two-dimensional (2-D) active (transmit and receive) sonar presentations.

Technological advances currently provide the capability to combine undersea data from a number of sensors including active sonars, video tape and electronic still cameras, manipulators, and laser range finders. These data can be assimilated and processed in real time to provide large quantities of high quality information. The data generated can be used to provide the ROV pilot with a real-time, three-dimensional (3-D), perspective display similar to that of the video camera when beyond visual range. This more natural view would ease the pilots workload and improve the efficiency of work conducted beyond visual camera range. Such a perspective-view display is expected to be dominated in the foreground by high-resolution video data, gradually transitioning to dominance by other sensors as the limits of video range are encountered. The envisioned 3-D display must be carefully designed to best improve the ROV pilot's efficiency.

\subsection{Research Objectives}

A variety of display enhancements have been used in military and commercial sonars. This thesis looks at the individual effects of six specific display enhancements: widening the field of view (FOV) to provide more information to the pilot, 2-D and 3-D gridding for improved angular and depth perception, vertical color schemes for better altitude versus depth perception, direct range readout to shorten the search and location cycle, and shortening the display update rate. Seven experiments to isolate and quantify the individual effects of these enhancements are described in detail. The analysis allows direct comparison of the effectiveness of the six enhancements. 


\subsection{Terminology}

Terminology is critical to comprehension. Several important definitions include:

- altitude - the distance above the floor of the ocean.

- data - "raw" numbers from which information can be extracted.

- data point - one of a set of $25 \mathrm{x}, \mathrm{y}$, and $\mathrm{z}$ coordinates chosen for a target course.

- depth - the perceived distance into a video or computer display. This is a spatial perception of range.

- display - the computer screen dedicated to graphics information.

- driving - the act of operating the simulated ROV for testing.

- heading - the compass direction in which the vehicle is pointed.

- monitor - the computer screen dedicated to printed information, including update indication,"target hit" and "mission complete" indication, target range indication (test 6), and vehicle world-coordinate position, heading, and velocities. This term is also used to describe the actions of the author as the testing supervisor.

- operator - a test subject who drove the simulated ROV.

- pilot - a qualified flyer of operational ROVs.

- piloting - the act of flying a operational ROV.

- target - the numbered, cubic wireframe, data point visualizing tool.

- target course - one of 19 (lettered A-R, X) specific sequences of data points and associated targets.

- update - the complete refreshing of a computer screen.

- vertical - the direction opposite to that in which gravity acts. A vertical color scheme encodes altitude information using a color scale or look-up table. 


\subsection{Overview}

Chapter 2 provides background information on human factors engineering and its relationship to the thesis work.

Chapter 3 contains information on three-dimensional computer graphics displays in general, and the display and equipment used in the thesis experiments.

Chapter 4 discusses current and projected applications of three-dimensional perspective displays in underwater visualization.

Chapter 5 describes in detail the conduct of seven tests including restricting the field of view, grids to provide depth and angular cues, color schemes to provide altitude cues versus depth of field, direct range information, and slowing of the graphic display update rate.

Chapter 6 presents the results of the seven tests including operator comments and author/test monitor observations.

Chapter 7 summarizes the results of the experiments, discusses conclusions, and offers recommendations for future work. 


\section{Chapter 2}

\section{Human Factors Engineering}

\subsection{Objectives and Doctrines}

Human factors engineering is the study of people and how equipment designs affect them. The two major objectives of human factors engineering are to improve efficiency and effectiveness and to promote key elements such as safety, satisfaction, and stress reduction. Six doctrines that form a set of human factors commandments are [S-1]:

- Design with the user in mind.

- Recognize differences in capabilities and limitations of people and their design implications.

- Design influences human behavior.

- Design process includes empirical data and evaluation.

- Reliance on scientific methods and objective data.

- Commitment to system orientation.

\subsection{Methodology Issues}

Experimental research methods test the effects of variables on behavior. Of prime interest in this work is the behavior "efficiency of operator performance". The method 
selected needed to accurately measure this efficiency. Eight important issues associated with the choice of methodology are [M-1]:

- Effectiveness.

- Ease of use.

- Cost.

- Flexibility.

- Range.

- Validity.

- Reliability.

- Objectivity.

No approach was best for all the issues above. Among them effectiveness, validity, reliability, and objectivity were deemed most critical for this thesis. Validity, in particular, was enhanced by input from experienced ROV pilots and operational support group personnel. These inputs served to more realistically match the simulation to operating conditions.

\subsection{Research Criteria}

Research criteria can be divided, with inevitable overlap, into the areas of systemdescriptive criteria, task-performance criteria, and human criteria [M-1]. The criteria chosen for analysis in this work was task performance, specifically performance time. Human criteria cannot be ignored, however, and provide insight in the form of subjective comments by the operators, both during and after each test, and observations by the test monitor/author. 


\subsection{Visual-Coding}

Color is a prime visual-coding technique. It has been frequently used in 3-D underwater visualization to provide depth/relief cueing in the form of a continuous color scale versus depth. In this work two discrete color sets were chosen for testing. Both the 6- and 21-color sets were derived from the red, green, and blue (RGB) color model. Figure 2.1 shows the RGB cube [F-1]. The 6-color set was selected by following a path from corner to corner in the following order: red, yellow, green, cyan, blue, and magenta. The 21-color set followed the same path, selecting colors at each 0.25 -unit path-length increment.

The designation of specific color names was complicated by the existence of a variety of descriptive systems. Among the more notable were RGB, HSB, Munsell, ISCC-NBS, YIQ, CIE, and UCL [B-1, F-1, K-1]. Target color names were derived starting with the basic six colors of the RGB color set (identical to the six basic hues of the HSB color set): red, yellow, green, cyan, blue, and magenta [F-1]. The further subdivisions were named in accordance with the spirit of the Color Naming System (CNS), which was developed to simplify color descriptions [B-1]. The 21 color names

derived were used for the 1-, 6-, and 21-color sets. Table 2.1 lists the colors by name and includes RGB and HSB descriptions. Conversions between most color sets were available and easily done with a computer program [F-1].

\subsection{Control and Tracking Performance}

Controls transmit discrete or continuous signals to a system to produce a desired response. Important factors in control design include ease of identification, size, controlresponse ratio, resistance, lag, deadspace, backlash, location, and compatibility [S-1]. 


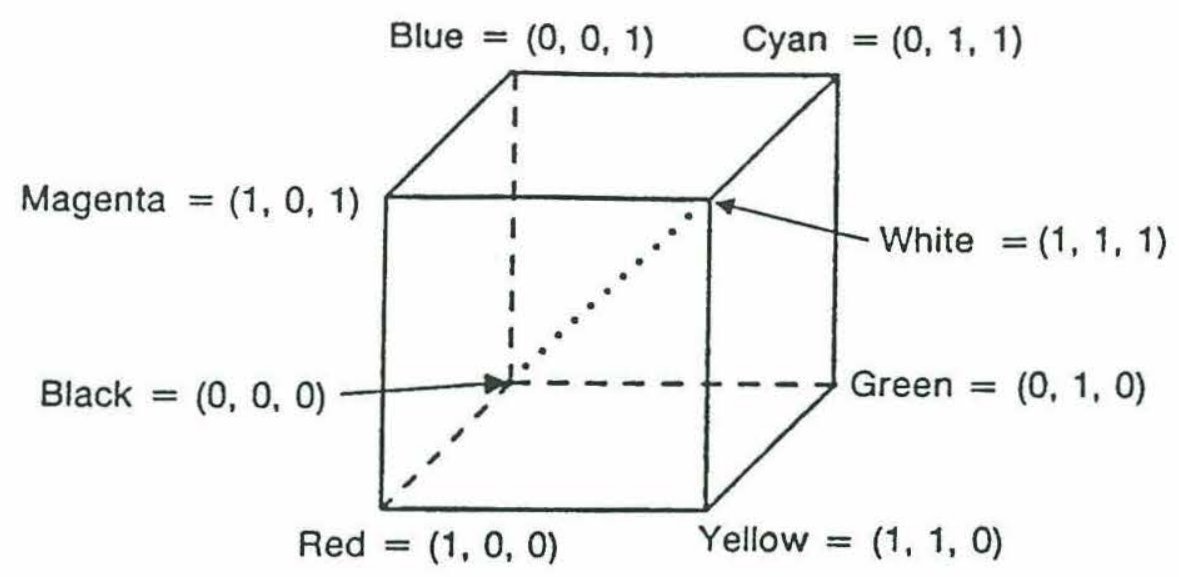

Figure 2.1 The RGB (red, green, and blue) cube [F-1]

Table 2.1 Color set RGB to HSB conversion

\begin{tabular}{|l|c|c|c|c|c|c|}
\hline \multicolumn{1}{|c|}{ Color } & Red & Green & Blue & Hue & Saturation & Brightness \\
\hline Red & 1 & 0 & 0 & 0 & 1 & 1 \\
\hline Reddish Orange & 1 & 0.25 & 0 & 15 & 1 & 1 \\
\hline Orange & 1 & 0.50 & 0 & 30 & 1 & 1 \\
\hline Yellowish Orange & 1 & 0.75 & 0 & 45 & 1 & 1 \\
\hline Yellow & 1 & 1 & 0 & 60 & 1 & 1 \\
\hline Yellowish Yellow-Green & 0.75 & 1 & 0 & 75 & 1 & 1 \\
\hline Yellow-Green & 0.50 & 1 & 0 & 90 & 1 & 1 \\
\hline Greenish Yellow-Green & 0.25 & 1 & 0 & 105 & 1 & 1 \\
\hline Green & 0 & 1 & 0 & 120 & 1 & 1 \\
\hline Greenish Aquamarine & 0 & 1 & 0.25 & 135 & 1 & 1 \\
\hline Aquamarine & 0 & 1 & 0.50 & 150 & 1 & 1 \\
\hline Cyanish Aquamarine & 0 & 1 & 0.75 & 165 & 1 & 1 \\
\hline Cyan & 0 & 1 & 1 & 180 & 1 & 1 \\
\hline Cyanish Turquoise & 0 & 0.75 & 1 & 195 & 1 & 1 \\
\hline Turquoise & 0 & 0.50 & 1 & 210 & 1 & 1 \\
\hline Blueish Turquoise & 0 & 0.25 & 1 & 225 & 1 & 1 \\
\hline Blue & 0 & 0 & 1 & 240 & 1 & 1 \\
\hline Blueish Purple & 0.25 & 0 & 1 & 255 & 1 & 1 \\
\hline Purple & 0.50 & 0 & 1 & 270 & 1 & 1 \\
\hline Magentaish Purple & 0.75 & 0 & 1 & 285 & 1 & 1 \\
\hline Magenta & 1 & 0 & 1 & 300 & 1 & 1 \\
\hline & & & & & & \\
\hline
\end{tabular}


Deadspace refers to a null established about the control's normal position in which control device motion results in no signal output. Backlash is deadspace at all control positions. The control mechanism may have no resistance (free-positioning or pure-displacement control), no displacement (stiff stick or pure-force control), or lie in the middle (which we term a mixture control).

The control order of a system refers to the relationship between the control mechanism and the system output. Orders of control include [S-1]:

- position (zero-order) control - where the signal controls the output directly.

- velocity (first-order) control - where the signal controls the rate of change of position.

- acceleration (second-order) control - where the signal controls the rate of change of velocity.

- higher-order control - where the signal controls the rate of change of acceleration or other higher order term.

The system in these tests used second-order control of thrust, equivalent to acceleration, in each of the four degrees of freedom (see Section 5.3).

The problem presented to the operator was a compensatory tracking problem with time lag [S-1]. The time lag was composed of three parts:

- response lag - the time required for the operator to choose and execute a response.

- control system lag - the time required for the system to respond to commands including vehicle motion simulation.

- display system lag - the time required for graphics calculations and display update.

In each case the time lag increased tracking error. 


\subsection{Test and Evaluation}

Independent variables (IVs) are those parameters controlled by the experimenter. Dependent variables (DVs) are measured parameters affected by the IVs. They are the same as the research criteria discussed in Section 2.3.

Evaluation of test results must consider test subjects, criteria, and experimental procedures [S-1]. Subjects should be representative of projected users. Dependent variables (criteria) must be related to system operational use. Experimental procedures and controls should give repeatable results. 


\section{Chapter 3}

\section{Three-Dimensional Computer Graphics Displays}

\subsection{True 3-D Displays}

Technology stands at the threshold of true three-dimensional displays. Available implementations display depth instead of simply providing depth cueing. Two such systems are $\underline{\text { SpaceGraph }}{ }^{\mathrm{TM}}$ by Bolt, Beranek, and Newman (BBN) Laboratories Incorporated of Cambridge, Massachusetts and $\underline{\text { mniView }}^{\mathrm{TM}}$ by Texas Instruments (TI) of Dallas, Texas.

The SpaceGraph ${ }^{\mathrm{TM}}$ system is based on reflecting a point plotted (not raster scanned) image in a vibrating mirror. The technique is termed the varifocal mirror. As the reflecting surface vibrates it repeatedly changes shape from concave to convex, causing a large change in focal length. When the image update rate is synchronized with the mirror vibration, a true 3-D image is created in space. For many data-set analyses the superior pattern-recognition capability of the human brain is enhanced by this type of display. This system is limited in viewing angle, display update rate, and to simple monochrome images. [F-1, T-1, S-2]

The OmniView $^{\mathrm{TM}}$ system is based on laser scanning of a paraboloidal, rapidly rotating surface. During rotation, the scanning laser beam's reflections form the 3-D image. Advantages include $360^{\circ}$ horizontal viewing and total wrap-around parallax. This system has no hidden surface removal and cannot display solid surfaces. [E-1] 
Holography is another promising technology for true 3-D viewing. This work is based on the splitting of a laser beam into two parts, illumination of objects by one of the split beams, and their subsequent recombination using the second beam as a reference. Limitations typically include small size and restricted viewing angle, lack of parallax, and high cost. [E-1, F-1]

Head-mounted displays have offered tremendous potential since their birth in the late 1960s [S-3, S-4]. The helmeted user enjoys freedom of movement in a virtual 3-D world projected in real time for separate viewing by the each eye. Technical problems have severely restricted the development of this technology since its inception [F-1].

An inherent limitation of true three-dimensional viewing is the tremendous quantity of data and associated data processing, particularly critical for real-time display. Expenses for computation rise dramatically as realistic, complex 3-D imagery is approached and parallel-processing supercomputers become a necessity. Significant effort and funding are being funnelled into this cutting-edge technology, but it is not likely to be available to the field scientist before the late 1990s.

\subsection{Simulated 3-D Displays}

In the absence of true 3-D displays several methods are employed to simulate threedimensional viewing using two-dimensional CRTs. These methods are designed to counter the information loss inherent in projecting 3-D information on a 2-D surface such as a computer screen. Techniques used include those for line drawings and shaded images, as well as dynamics, stereopsis, and head-motion parallax.

Line drawing techniques include the use of various projections, depth cueing with intensity, depth clipping, and hidden-line removal [F-1]. Among the projections, the perspective offers two significant depth cues: decreasing size of objects with depth and the 
convergence of parallel lines to their vanishing points. Depth cueing with intensity draws the more distant objects at a lower intensity. The result is a strong depth cue similar to that observed when viewing a scene in a light fog. Depth clipping limits the view by preventing the drawing of lines not within the designated limits of the front or back clipping planes. This effect is used to limit the depth of field. Hidden line removal calculates the lines that should not be visible based on the view and does not draw them.

Shaded-image techniques include hidden-surface removal, control of illumination and shading, various shading interpolation methods, and the use of texture, shadows, transparency, and reflection.

Dynamics refers to the added information available when a sequence of views is shown instead of a static view. The primary depth cue is provided by the kinetic depth effect, the lower velocity of nearby objects versus those in the background.

Stereopsis is a depth cue taking advantage of the binocular disparity between human eyes [F-1]. The ability of the human brain to fuse two slightly differing views of a scene and extract depth information is a powerful depth cue.

Head-motion parallax uses the changing view as occurs in real life when the head is moved side to side.

\subsection{The Marquest System}

Several important components of the test equipment hardware and software were provided by the Marquest Group, Inc. of Bourne, Massachusetts. They include the

joystick module and associated software, an Nth Engine/550 ${ }^{\mathrm{TM}}$ Display Controller, and HOOPS $^{\mathrm{TM}}$ graphics routines and HYDRA ${ }^{\mathrm{TM}}$ rendering software for the Nth Engine/550 ${ }^{\mathrm{TM}}$. They are components of a commercial ROV control system.

The joystick module is a standardized control device for ROVs constructed by the Marquest Group, Inc. using off-the-shelf components. Included are a trackball, bias and 
gain potentiometers, nine push-button switches, and a 3-axis joystick. Only the joystick was used in the testing. Measurement Systems, Inc. of Norwalk, Connecticut, built the industry standard, heavy duty, 3 -axis $(x, y, \theta)$ joystick with mixture control and deadspace on each of the three axes. A thumb switch provided the fourth axis of freedom (z) with pure force control. Another joystick available from Measurement Systems, Inc., but not used here, incorporated the fourth axis of freedom through lifting/depressing the joystick vertically with mixture control. Joystick use is discussed in Chapters 6 and 7.

The Nth Engine/550 ${ }^{\mathrm{TM}}$ Display Controller is a 3-D graphics processor/controller built by Nth Graphics of Austin, Texas. The controller has $1280 \times 1024$ resolution and operates at 20 MIPS and 3 MFLOPS. The board set includes an NthTV ${ }^{\mathrm{TM}}$ converter module, which provides a standard commercial television format (NTSC) tap for direct, real-time recording of the video display on a video cassette recorder (VCR).

HOOPS $^{\mathrm{TM}}$ from Ithaca Software of Ithaca, New York, formed an interactive threedimensional graphics library. This software controlled the Nth Engine $/ 550^{\mathrm{TM}}$.

HYDRA $^{\mathrm{TM}}$ rendering software, another product of Nth Graphics, was used to convert targets and grids drawn in AutoCAD ${ }^{\circ}$ Release 10 to the required format for the HOOPS graphics routines. A helpful feature is the ability to quickly conduct a visual check of targets and grids.

\subsection{The Display and the Monitor}

Planar geometric projections fall into two categories: perspective and parallel. Characteristics which distinguish a perspective projection from a parallel projection are convergence of parallel lines, size diminution, and nonuniform foreshortening [C-1]. The primary advantage of a perspective projection is the natural appearance of objects seen by the eye. It was also the standard video camera projection. For these reasons a three-point 
perspective projection was chosen for the display [C-1]. The graphics were shown on a Sony model GDM-1950 19-inch color monitor with 1280 x 1024 pixel resolution.

Of the previously discussed techniques to provide three dimensionality, depth cueing in particular, many were not available in the experimental system for three reasons: the tests used a perspective projection, the targets were drawn in wireframe mode, and limitations of the Nth Graphics boards and software running the display. Depth cueing was provided by target relative size (because they were all identical in dimension), the convergence of parallel lines (emphasized by the cubic wireframe targets), and the kinetic depth effect.

An NEC VGA color monitor (with blue background) provided the following:

- display update indicated by the word "Busy.." appearing in white letters in a red box.

- most recent target hit indicated by the words "OBJECT \#(target number) HIT" appearing in white letters in a red box.

- test completion indicated by the words "MISSION COMPLETE" appearing in white letters in a red box.

- range in meters from the vehicle (modelled as a point) to the center of the next target indicated by the symbol " $\mathrm{R}=$ (range)" appearing in white letters in a red box. This information was provided to the operator only during test 6 .

- world system coordinates ( $\mathrm{x}, \mathrm{y}$, and $\mathrm{z})$ and heading of the vehicle in white numerals. Titles (xpos, ypos, zpos, and hdg) were shown beside the coordinates/heading in white letters.

- vehicle system velocities ( $\dot{x}, \dot{y}, \dot{z}$, and $\theta$ rate) in white numerals. The titles (xvel, yvel, zvel, and rvel) shown in black letters above the velocities were individually accented with small red boxes if their magnitudes exceeded $0.05 \mathrm{~m} / \mathrm{s}$ or $0.05 \mathrm{rad} / \mathrm{s}$.

Figure 3.1 shows the monitor screen with range information during test 6 . 


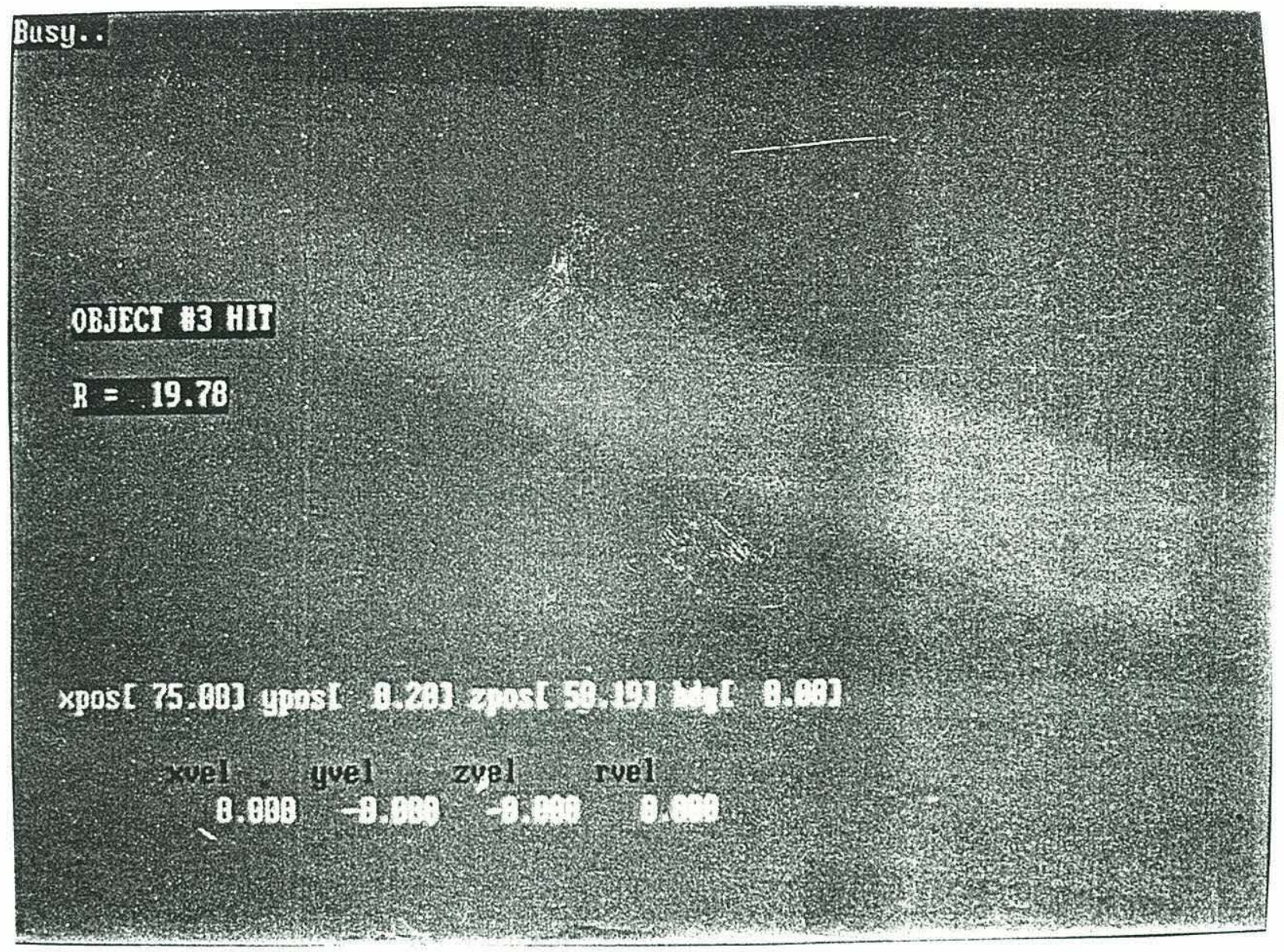

Figure 3.1 Monitor screen during test 6 


\section{Chapter 4}

\section{Application of the 3-D Perspective Display}

\subsection{Ocean Environmental Sensing}

Early sonar development was driven by military needs. Crude processing and display techniques limited these systems to deterministic methods with severely restricted dynamic range. Inherent in these limitations were a disregard for inaccuracies such as angular and range resolution, variations in the speed of sound and the sound ray paths, and navigation errors in position, attitude, depth and motion. The current state of technology overcomes many of these restrictions. Modern computers and software graphics packages allow real-time processing and display of more advanced models of the ocean environment. New commercial sonars provide more accurate information from a variety of sampling techniques including swath mapping, sidescan, and upward-looking sonars, in addition to the more traditional forward-looking and bathymetric sounding versions. Innovative electronic instruments provide high-accuracy navigational data. Recent trends have resulted in additional technologies being adapted for underwater work. Video and electronic still cameras, and scanning lasers are among these supplementary sensors. 


\subsection{Multisensor Modeling}

To effectively combine the various sensor modalities into a single model of the ocean environment, we must first accept the premise that no measurement is exact. Any information gathered will have an associated probability distribution, which is itself not known deterministically. The ocean model can be built incorporating these uncertainties as a stochastic model. This approach is especially effective when combining information from different sources with their differing ranges, resolutions, and data types. At any time, a deterministic model can be extracted from the current probabilistic one. Using this method, a more accurate deterministic model is obtained which accounts for the many and varied errors inherent in measurements.

Initially, the ocean volume of interest is divided into a 3-D grid of cubic volume elements, or voxels. The size of the voxels is dependent upon the desired model resolution as well as system hardware and software limitations for real-time processing. Each voxel is assigned a "feature" vector incorporating information such as the time, the position of the voxel in the coordinate space, the measured characteristics of interest (features), and a probability or confidence value for each feature. As new information is collected, older data is not discarded. Feature vectors are incremented using a data merging technique called stochastic backprojection [S-5, S-6]. This data merging technique is an adaptation of the backprojection and summation method used to reconstruct images. The method is enhanced to include probabilistic uncertainty and allow stepwise solutions for real-time processing. Use of these techniques allows combination of the redundant feature data from all available sources, improving the model resolution and certainty. 


\subsection{The Deep Submergence Laboratory System}

The Deep Submergence Laboratory of the Woods Hole Oceanographic Institute employs a variety of sensors and platforms. A typical cruise involves flying equipment to a proximate port and loading out a chartered mother vessel. At the research site, a large area survey is conducted with a towed $120-\mathrm{kHz}$ split-beam sonar system. This survey provides medium resolution imaging with a swath width of 200-300 meters at a tow speed of 6-10 knots, and allows items of interest to be identified for subsequent revisiting with higher resolution equipment. Items of interest are reinspected using the tethered Jason ROV system. Jason is typically equipped with a SHARPS short-baseline navigation system and a sensor package including a $200-\mathrm{kHz}$ sidescan sonar, a $300-\mathrm{kHz}$ forward-scanning sonar, a video camera, an electronic still camera, and a laser rangefinder. Figure 4.1 shows the range and resolution overlaps between sensors in the current suite.

The Jason pilot has several sources of information available. If within visual range, the video camera and vehicle lighting system provide a natural, perspective view with extremely high bandwidth. This visual range is often restricted to a meter or less due to suspended sediment in the water, and is usually less than about 10 meters. Beyond visual range the forward-scanning sonar, the $200-\mathrm{kHz}$ sidescan sonar, the laser rangefinder, and the SHARPS navigation system provide data to several displays with differing formats. The pilot navigates using a specially designed 2-D auxiliary display with limited bandwidth until within visual range [S-7].

This current method taxes the pilot, whose task is complicated by [S-7]:

- low visibility, reducing video camera range and safe working speeds.

- the narrow field of view of the video camera (approximately $40^{\circ}$ ).

- the monocular vision of the video camera.

- optical distortion of the video camera.

- the difficulty of interpreting sonar presentation screens. 


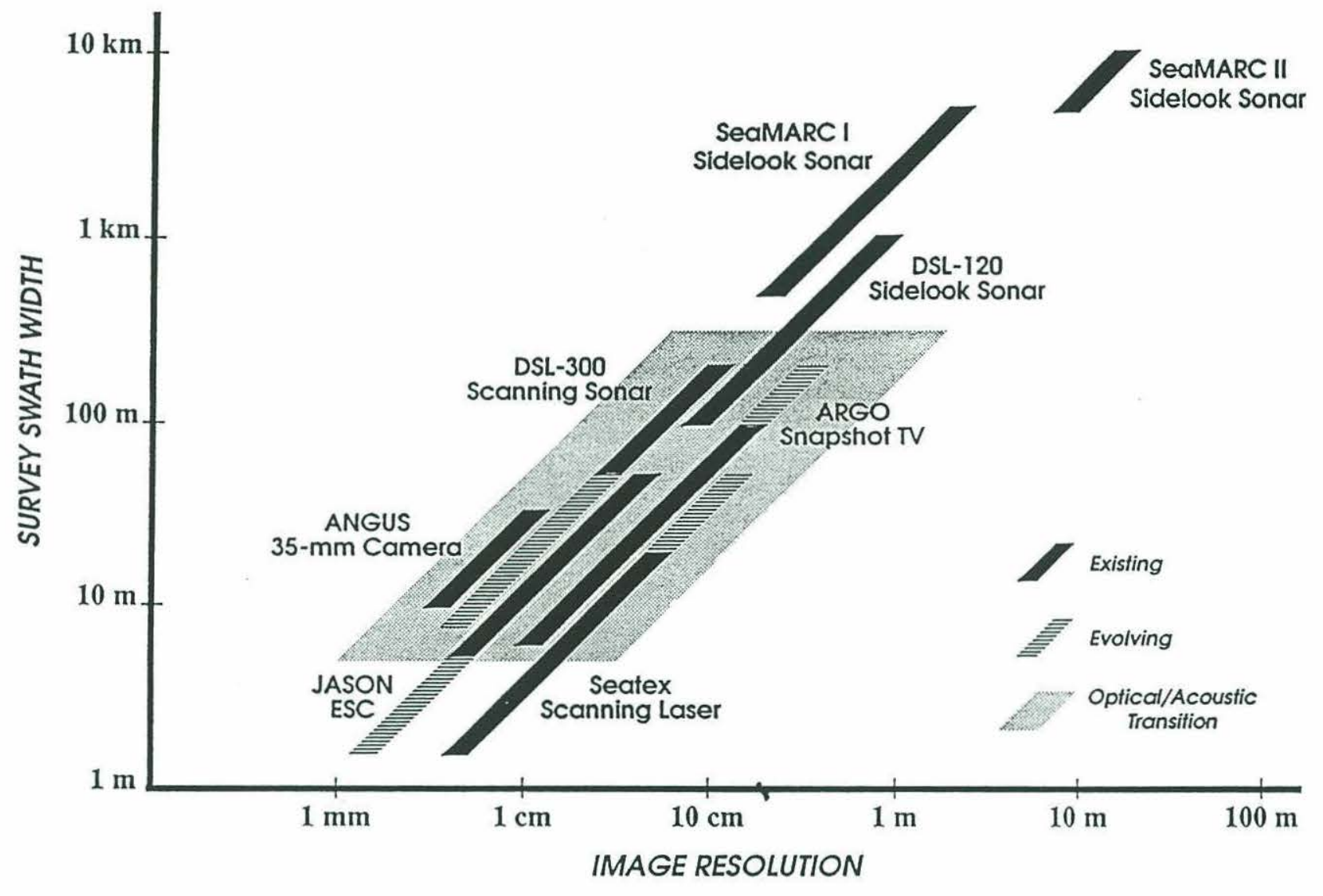

Figure 4.1 DSL comparison of underwater remote-sensing systems 
- the transient nature of the displayed information, requiring the pilot to mentally integrate the overall picture.

- the inability to access the high-level, real-time environmental model.

To enhance ROV piloting efficiency, a real-time, 3-D display is envisioned. The display will access the high-level environmental model and present the user with a view closely resembling that of the video camera. Because low or medium resolution data are available prior to the ROV entering the water, a rough perspective-view display can be constructed at any time for use by the pilot on the screen previously reserved for video camera information. Such a perspective-view display is expected to be dominated in the foreground by high-resolution video data, gradually transitioning to dominance by other sensors as the limits of video range are encountered.

\subsection{Targeted Human Factors}

Among the myriad of potential display enhancements to improve the proposed 3-D perspective display are:

- altering the displayed field of view.

- providing a screen grid.

- displaying current range to the target of interest.

- use of a vertical color scheme.

- controlling the display update rate.

Narrowing the displayed field of view reduces the necessary graphics calculations as the square of the reduction factor. This gain must be balanced against the loss of information to the user. 
Providing a 2-D or 3-D screen grid can improve the user's angular perception, provide a cross-hair effect to ease direction finding and, with the 3-D version, provide important depth cues.

Displaying current range to the target of interest can reduce the time to locate the target. This information is available in the environmental model.

Vertical color schemes are frequently used to provide redundant relief cueing for underwater and land feature presentations such as charts and maps. They can be effective in giving altitude cues for objects in the background of perspective displays.

Display update rate is limited by the data-collection cycle time of the sensors, data processing time, and graphics calculation/display time. Compromises can be made to shorten the update rate at the cost of information quality and quantity.

This thesis attempts to quantify the effects of the task-related independent variables listed above. The dependent variables for the experiments are performance time, subjective comments by the operators, and observations by the test monitor/author. 


\section{Chapter 5}

\section{Testing Display Effectiveness}

\subsection{Equipment}

Three system hardware components were used directly by the operator: a computer monitor, a computer display, and a joystick. Other equipment transparent to the operator's task included a 386 computer with keyboard and mouse, which ran the simulation and recorded the data, and various power supply and communications cabling.

\subsection{Data Point Courses}

Nineteen data courses (A-R, X) were used in testing. Courses consisted of 25 sequentially numbered data points $\left(\mathrm{P}_{\mathrm{j}}\right)$ in world coordinate system three space $(\mathrm{x}, \mathrm{y}, \mathrm{z})$ with two constraints:

(1) Consecutive points were separated by distance $s=\sqrt{[\Delta x]^{2}+[\Delta y]^{2}+[\Delta z]^{2}}$, where $\mathrm{s}$ was a constant equal to 5 meters.

(2) A square window of given total angular width and height $\beta$, with apex at point $\mathrm{P}_{\mathrm{j}}$ and opening in the direction of a line from $\mathrm{P}_{\mathrm{j}-1}$ through $\mathrm{P}_{\mathrm{j}}$, contained point $P_{j+1}$.

Courses A, B, C and J, K, L used a $15^{\circ}$-square window, courses D, E, F and M, N, O used a $30^{\circ}$ window, and courses $\mathrm{G}, \mathrm{H}, \mathrm{I}$ and $\mathrm{P}, \mathrm{Q}, \mathrm{R}$ used a $60^{\circ}$ window. Course $\mathrm{X}$, the training/familiarization course, used a $15^{\circ}$ window. Additionally, nine of the courses were 
mirror images of each other in the world coordinate system $y / z$ plane. Mirror pairs were $\mathrm{A} / \mathrm{J}, \mathrm{B} / \mathrm{K}, \mathrm{C} / \mathrm{L}, \mathrm{D} / \mathrm{M}, \mathrm{E} / \mathrm{N}, \mathrm{F} / \mathrm{O}, \mathrm{G} / \mathrm{P}, \mathrm{H} / \mathrm{Q}$ and $\mathrm{I} / \mathrm{R}$. Figure 5.1 and table 5.1 summarize the above information.

Visualization of each data point was provided in the form of a cubic wireframe target one meter on a side with the invisible data point at its center. Target faces were parallel to the planes formed by the world coordinate system axes. Target color was blue except for tests 5 and 6 as discussed in section 5.7. Each target was numbered with white numerals (1-25) corresponding to the sequential number of the data point at its center. A target "hit" corresponded to closing the range to the target center/data point to 0.5 meters or less from any direction. Appendix $\mathrm{C}$ discusses the details of target design. Appendix D contains course layout data and photographs of the courses as viewed by the operator.

Random generation of the data points for each course began with establishment of two coordinate systems, the world and the vehicle. Each system consisted of three mutually perpendicular axes $\mathrm{x}, \mathrm{y}$, and $\mathrm{z}$, forming a right-hand coordinate system. The world system was fixed in space. In it, three angles were defined: $\omega, \varphi$, and $\kappa$ for positive rotation about the $\mathrm{x}, \mathrm{y}$ and $\mathrm{z}$ axes respectively. Positive rotation was defined such that positive $\omega(\varphi, \kappa)$ rotated the positive $\mathrm{y}(\mathrm{z}, \mathrm{x})$ axis into the positive $\mathrm{z}(\mathrm{x}, \mathrm{y})$ axis through $90^{\circ}$. In the vehicle system, the operator was assumed to be looking down the positive $y$ axis of the system at all times with the positive $\mathrm{x}$ axis to the right. Positive rotation was defined so positive $\theta$ rotated the positive $y$ axis into the positive $\mathrm{x}$ axis through $90^{\circ}$. In this way, $\theta$ had the same sense as true heading. Figures 5.2 and 5.3 depict these systems. For a specified window width $\beta$, sets of two angles $\omega$ and $\mathrm{k}$ were randomly selected from the interval $[-\beta / 2, \beta / 2]$. This process insured that the data points met constraint (2) above.

Based on the above coordinate system definitions, rotations of the vehicle system were broken down into individual rotations of $\omega, \varphi$, and $\kappa$. Orthogonal orientation matrices were then defined for the decomposition as follows [M-2]: 


$$
\begin{aligned}
& M_{\omega}=\left[\begin{array}{ccc}
1 & 0 & 0 \\
0 & \cos \omega & \sin \omega \\
0 & -\sin \omega & \cos \omega
\end{array}\right] \\
& M_{\varphi}=\left[\begin{array}{ccc}
\cos \varphi & 0 & -\sin \varphi \\
0 & 1 & 0 \\
\sin \varphi & 0 & \cos \varphi
\end{array}\right] \\
& M_{\kappa}=\left[\begin{array}{ccc}
\cos \kappa & \sin \kappa & 0 \\
-\sin \kappa & \cos \kappa & 0 \\
0 & 0 & 1
\end{array}\right]
\end{aligned}
$$

The most general orientation matrix $\mathrm{M}$ for any combination of these three rotations was then $[\mathrm{M}-2]$ :

$$
\begin{aligned}
M & =M_{\omega} M_{\varphi} M_{\kappa} \\
= & {\left[\begin{array}{rr}
\cos \varphi \cos \kappa & \cos \omega \sin \kappa+\sin \omega \sin \varphi \cos \kappa \\
-\cos \varphi \sin \kappa & \cos \omega \cos \kappa-\sin \omega \sin \varphi \sin \kappa \\
\sin \varphi & -\sin \omega \cos \varphi \\
\sin \omega \sin \kappa-\cos \omega \sin \varphi \cos \kappa \\
\sin \omega \cos \kappa+\cos \omega \sin \varphi \sin \kappa \\
\cos \omega \cos \varphi
\end{array}\right.}
\end{aligned}
$$

In our application pitch $(\omega)$ and yaw $(\kappa)$ were allowed to vary while roll $(\varphi)$ was not. The general case was then specialized by setting $\varphi=0$ to obtain:

$$
L=M_{\varphi=0}=\left[\begin{array}{ccc}
\cos \kappa & \cos \omega \sin \kappa & \sin \omega \sin \kappa \\
-\sin \kappa & \cos \omega \cos \kappa & \sin \omega \cos \kappa \\
0 & -\sin \omega & \cos \omega
\end{array}\right]
$$

The matrix $L_{j}$ defined the look direction from the point $P_{j}$ to the next point $P_{j+1}$ in the vehicle coordinate system. The matrix $\left(\mathrm{L}_{\mathrm{j}}\right)^{*}$ defined the transformation from the vehicle 
coordinate system to that of the world. The origin point was taken to be $\mathrm{P}_{0}=[0,0,0]^{\mathrm{T}}$. The first data point was taken to be $\mathrm{P}_{1}=\mathrm{s}[0,1,0]^{\mathrm{T}}$, where $\mathrm{s}$ was the constant described in constraint (1). The value $(0,0)$ was assigned to the set $(\omega, \kappa)$ at $\mathrm{x}=0$ yielding $\mathrm{L}_{0}=\mathrm{I}_{3 \times 3}$, the three-dimensional identity matrix, and successive $L_{j} s$ were generated using the above formula for matrix $\mathrm{L}$ and the $\mathrm{j}$-th set of values $(\omega, \kappa)$. As a result of these specifications $\left(\mathrm{L}_{0}\right)^{*}=\mathrm{I}_{3 \times 3}$ and successive $\left(\mathrm{L}_{\mathrm{j}}\right)^{*}$ s were calculated from the equation $\left(\mathrm{L}_{\mathrm{j}+1}\right)^{*}=\left(\mathrm{L}_{\mathrm{j}}\right)^{*} \mathrm{~L}_{\mathrm{j}}$. Successive data points were then generated using $\mathrm{P}_{\mathrm{j}+1}=\mathrm{P}_{\mathrm{j}}+\mathrm{s}\left(\mathrm{L}_{\mathrm{j}}\right)^{*} \mathrm{~L}_{\mathrm{j}}[0,1,0]^{\mathrm{T}}$ as the recursive algorithm.

\subsection{Modeling the Jason Vehicle}

The vehicle model was a dynamic simulation of the DSL ROV Jason. The model exhibited four degrees of freedom: $x$ (left/right), y (forward/reverse), $z$ (up/down), and $\theta$ (CW/CCW). Hydrodynamic drag was assumed to have quadratic and linear (damping) components. Balancing forces yielded the equation (shown for the $\mathrm{x}$ component) :

$$
\left(M+M_{a}\right) \ddot{x}+\left[\frac{\left(M+M_{a}\right)}{\tau_{\max }}\right] \dot{x}+C_{D} \dot{x}|\dot{x}|=T
$$

where,

M was the vehicle's mass, $M_{a}$ was the vehicle's added mass, and $\left(M+M_{a}\right)$ was termed the vehicle's extended mass.

$\tau_{\max }$ was a constant $(2.0 \mathrm{~s})$ defining the magnitude of the linear drag.

$\mathrm{CD}_{\mathrm{D}}$ was the drag coefficient.

$\mathrm{T}$ was the maximum thrust.

Table 5.2 summarizes the model parameters use in the experiment. Nonlinear thruster dynamics were not included in the vehicle simulation model. 
The square window actually defined a portion of the surface of a sphere because point $P_{j+1}$ was required to be exactly distance $s$ from point $\mathrm{P}_{\mathrm{j}}$.

Figure 5.1 Visualization of data point course constraints

Table 5.1 Data point course relationships

\begin{tabular}{|c|c|c|c|c|c|c|c|c|c|c|}
\cline { 2 - 11 } \multicolumn{1}{c|}{} & \multicolumn{4}{c|}{$15^{\circ}$ Solid Angle } & \multicolumn{4}{c|}{$30^{\circ}$ Solid Angle } & \multicolumn{3}{c|}{$60^{\circ}$ Solid Angle } \\
\hline Image & $\mathrm{A}$ & $\mathrm{B}$ & $\mathrm{C}$ & & $\mathrm{D}$ & $\mathrm{E}$ & $\mathrm{F}$ & $\mathrm{G}$ & $\mathrm{H}$ & $\mathrm{I}$ \\
\hline Mirror & $\mathrm{J}$ & $\mathrm{K}$ & $\mathrm{L}$ & & $\mathrm{M}$ & $\mathrm{N}$ & $\mathrm{O}$ & $\mathrm{P}$ & $\mathrm{Q}$ & $\mathrm{R}$ \\
\hline Training & & & & $\mathrm{X}$ & & & & & & \\
\hline
\end{tabular}

Table 5.2 Dynamic vehicle simulation parameters

\begin{tabular}{|c|c|c|c|c|}
\hline & $\begin{array}{c}\text { Extended Mass } \\
(\mathrm{kg})\end{array}$ & $\begin{array}{c}\text { Drag Coefficient } \\
(\mathrm{kg} / \mathrm{m} \text { or } \mathrm{kg}-\mathrm{m})\end{array}$ & $\begin{array}{c}\text { Max Thrust } \\
(\mathrm{N})\end{array}$ & $\begin{array}{c}\text { Max Velocity } \\
(\mathrm{m} / \mathrm{s} \text { or rad/s })\end{array}$ \\
\hline $\mathrm{x}$ & 1800 & 400 & 250 & 0.25 \\
\hline $\mathrm{y}$ & 1350 & 350 & 250 & 0.50 \\
\hline $\mathrm{z}$ & 1800 & 400 & 250 & 0.25 \\
\hline$\theta$ & 450 & 250 & 41.5 & 0.157 \\
\hline
\end{tabular}




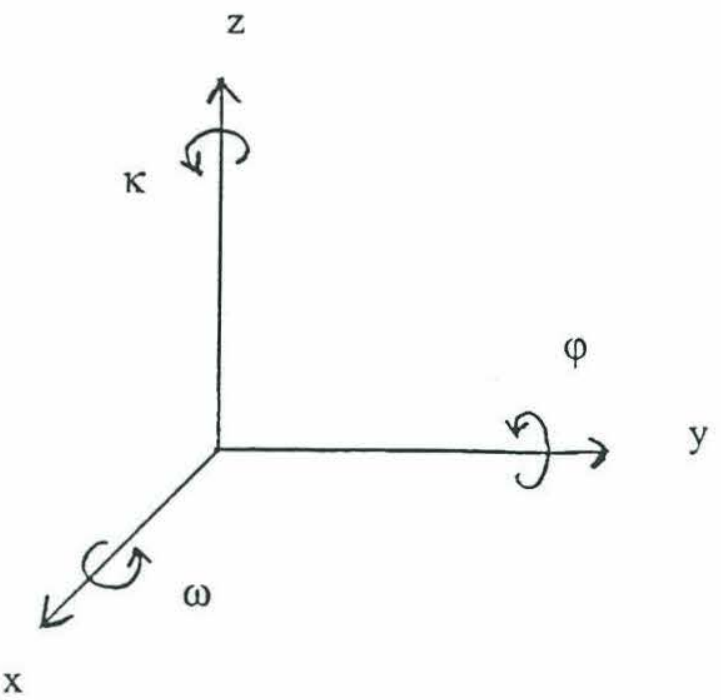

Figure 5.2 The world coordinate system

z

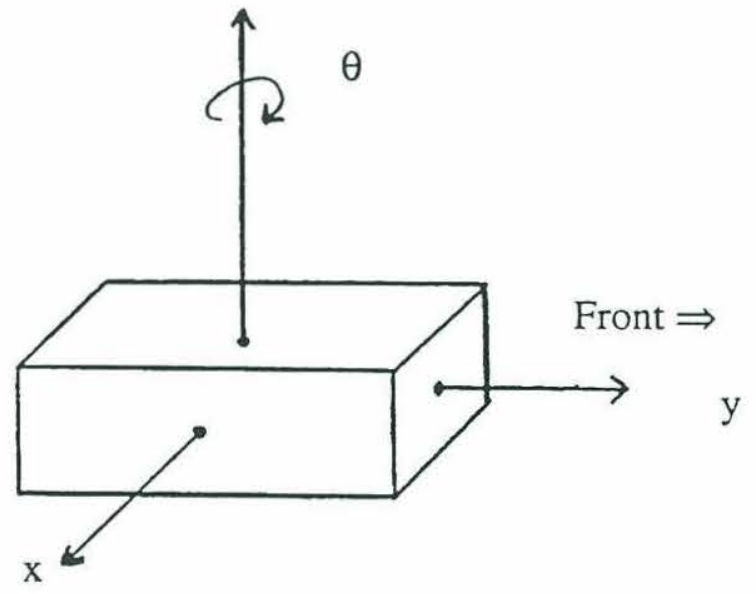

Figure 5.3 The vehicle coordinate system 


\subsection{Operators and Training}

The same four operators were used in each of the seven tests. The author was not an operator. Operator profiles were:

Operator I was a 22 year old, male Naval Officer and graduate student with uncorrected full visual acuity and normal color vision. This right-handed subject had frequently played video games including flight simulators. He was familiar with 3-D perspective computer displays prior to the experiment.

Operator II was a 24 year old, male graduate student with uncorrected full visual acuity and normal color vision. This left-handed subject had little exposure to video games such as flight simulators. He was familiar with 3-D perspective computer displays prior to the experiment due to practical experience with computer systems as an undergraduate Computer Science major.

Operator III was a 34 year old, male Naval Officer and graduate student with uncorrected full visual acuity and normal color vision. This right-handed subject had some exposure to video games such as flight simulators. He was familiar with 3-D perspective computer displays prior to the experiment.

Operator IV was a 32 year old male with a Master's Degree, uncorrected full visual acuity, and normal color vision. This right-handed subject had little exposure to video games such as flight simulators. He was not familiar with 3-D perspective computer displays prior to the experiment.

Prior to each testing session, operators were briefed in the following areas: 
general, simulation, and hardware. The general topics included purpose of thesis and experimental approach, time measurement, and operator errors. Simulation topics included target and course design as well as vehicle modeling. Hardware subjects discussed important features of the computer monitor, the computer display, and the joystick. Additionally, a maximum of 15 minutes was allotted for familiarity training with a $60^{\circ}, 30^{\circ}$, or $15^{\circ}$ field of view, as appropriate to the test, using training course $\mathrm{X}$.

The tests were conducted in a total of four separate sittings to reduce operator fatigue. Test 1 (see number designations below) was conducted alone. The remaining tests were conducted in pairs in the following order: $2 / 3,4 / 5$, and $6 / 7$.

\subsection{Test 1 - Field of View}

Testing for field of view measured changes in the operator's spatial performance versus the displayed field of view.

Test 1 description: All targets were colored blue. The operators drove the simulated vehicle point to point over a course of 25 data points spaced in $\mathrm{x}, \mathrm{y}$, and $\mathrm{z}$. Operators completed nine courses, each varying in $x / y / z$ with a maximum $15^{\circ}, 30^{\circ}$, or $60^{\circ}$ spread in data from point to point. Measured fields of view were $15^{\circ}, 30^{\circ}$, and $60^{\circ}$. The display update period was fixed at two seconds. Nine combinations of FOV and courses were used with each of the four operators.

\subsection{Tests 2 and 3 - Gridding}

Testing for gridding measured the improvement in operator performance when 
presented with a visual reference grid in the display.

Test 2 description: All targets were colored blue. The operators drove the simulated vehicle point to point over a course of 25 data points spaced in $\mathrm{x}, \mathrm{y}$, and $\mathrm{z}$. Operators completed three courses, each varying in $x / y / z$ with a maximum $15^{\circ}, 30^{\circ}$, or $60^{\circ}$ spread in data from point to point. The field of view was fixed at $60^{\circ}$ and the display update period at two seconds. A two-dimensional (2-D) grid was superimposed on the visual display.

Test 3 description: All targets were colored blue. The operators drove the simulated vehicle point to point over a course of 25 data points spaced in $\mathrm{x}, \mathrm{y}$, and $\mathrm{z}$. Operators completed three courses, each varying in $x / y / z$ with a maximum $15^{\circ}, 30^{\circ}$, or $60^{\circ}$ spread in data from point to point. The field of view was fixed at $60^{\circ}$ and the display update period at two seconds. A three-dimensional (3-D) grid was superimposed on the visual display.

\subsection{Tests 4 and 5 - Vertical Color Schemes}

Testing for color scheme measured the improvement in operator performance using a fixed, vertical-relief color scheme for target shading versus single-color targets. The 6color set provided 12 meter vertical color swaths while the 21 -color set provided three meter vertical color swaths over the range of interest. Table 5.3 lists the color sets.

Test 4 description: Targets were shaded in color according to height $\mathrm{z}$ above ocean floor. The color scheme was divided into six discrete shades. The operators drove the simulated vehicle point to point over a course of 25 data points spaced in $\mathrm{x}, \mathrm{y}$, and $\mathrm{z}$. Operators completed three courses, each varying in $\mathrm{x} / \mathrm{y} / \mathrm{z}$ with a maximum $15^{\circ}, 30^{\circ}$, or $60^{\circ}$ spread in 
Table 5.3 The color sets $(1,6,21)$ and associated altitude bands

\begin{tabular}{|c|c|l|c|c|c|c|}
\hline Set & Target & \multicolumn{1}{|c|}{ Color } & Red & Green & Blue & Z value (meters) \\
\hline 1 & 0 & Blue & 0 & 0 & 1 & All \\
\hline 6 & 1 & Red & 1 & 0 & 0 & $<88$ \\
\hline 6 & 2 & Yellow & 1 & 1 & 0 & $76-88$ \\
\hline 6 & 3 & Green & 0 & 1 & 0 & $64-76$ \\
\hline 6 & 4 & Cyan & 0 & 1 & 1 & $52-64$ \\
\hline 6 & 5 & Blue & 0 & 0 & 1 & $40-52$ \\
\hline 6 & 6 & Magenta & 1 & 0 & 1 & $<40$ \\
\hline 21 & 7 & Red & 1 & 0 & 0 & $>93$ \\
\hline 21 & 8 & Reddish Orange & 1 & 0.25 & 0 & $90-93$ \\
\hline 21 & 9 & Orange & 1 & 0.50 & 0 & $87-90$ \\
\hline 21 & 10 & Yellowish Orange & 1 & 0.75 & 0 & $84-87$ \\
\hline 21 & 11 & Yellow & 1 & 1 & 0 & $81-84$ \\
\hline 21 & 12 & Yellowish Yellow-Green & 0.75 & 1 & 0 & $78-81$ \\
\hline 21 & 13 & Yellow-Green & 0.50 & 1 & 0 & $75-78$ \\
\hline 21 & 14 & Greenish Yellow-Green & 0.25 & 1 & 0 & $72-75$ \\
\hline 21 & 15 & Green & 0 & 1 & 0 & $69-72$ \\
\hline 21 & 16 & Greenish Aquamarine & 0 & 1 & 0.25 & $66-69$ \\
\hline 21 & 17 & Aquamarine & 0 & 1 & 0.50 & $63-66$ \\
\hline 21 & 18 & Cyanish Aquamarine & 0 & 1 & 0.75 & $60-63$ \\
\hline 21 & 19 & Cyan & 0 & 1 & 1 & $57-60$ \\
\hline 21 & 20 & Cyanish Turquoise & 0 & 0.75 & 1 & $54-57$ \\
\hline 21 & 21 & Turquoise & 0 & 0.50 & 1 & $51-54$ \\
\hline 21 & 22 & Blueish Turquoise & 0 & 0.25 & 1 & $48-51$ \\
\hline 21 & 23 & Blue & 0 & 0 & 1 & $45-48$ \\
\hline 21 & 24 & Blueish Purple & 0.25 & 0 & 1 & $42-45$ \\
\hline 21 & 25 & Purple & 0.50 & 0 & 1 & $39-42$ \\
\hline 21 & 26 & Magentaish Purple & 0.75 & 0 & 1 & $36-39$ \\
\hline 21 & 27 & Magenta & 1 & 0 & 1 & $<36$ \\
\hline
\end{tabular}


data from point to point. The field of view was fixed at $60^{\circ}$ and the display update period at two seconds.

Test 5 description: Targets were shaded in color according to height $\mathrm{z}$ above ocean floor. The color scheme was divided into 21 discrete shades. The operators drove the simulated vehicle point to point over a course of 25 data points spaced in $\mathrm{x}, \mathrm{y}$, and $\mathrm{z}$. Operators completed three courses, each varying in $x / y / z$ with a maximum $15^{\circ}, 30^{\circ}$, or $60^{\circ}$ spread in data from point to point. The field of view was fixed at $60^{\circ}$ and the display update period at two seconds.

\subsection{Test 6 - Heads Up Range}

Testing for range measured the improvement in operator performance when presented with continuously revised range to target center information.

Test 6 description: All targets were colored blue. The operators drove the simulated vehicle point to point over a course of 25 data points spaced in $\mathrm{x}, \mathrm{y}$, and $\mathrm{z}$. Operators completed three courses, each varying in $x / y / z$ with a maximum $15^{\circ}, 30^{\circ}$, or $60^{\circ}$ spread in data from point to point. The field of view was fixed at $60^{\circ}$ and the display update period at two seconds. Continuously revised range to target center was provided to the operator.

\subsection{Test 7 - Display Update Rate}

Testing for display update rate measured the degradation in operator performance associated with reduced visual information feedback. 
Test 7 description: All targets were colored blue. The operators drove the simulated vehicle point to point over a course of 25 data points spaced in $\mathrm{x}, \mathrm{y}$, and $\mathrm{z}$. Operators completed three courses, each varying in $x / y / z$ with a maximum $15^{\circ}, 30^{\circ}$, or $60^{\circ}$ spread in data from point to point. The field of view was fixed at $60^{\circ}$. The display update period was slowed to four seconds. 


\section{Chapter 6}

\section{Testing Results}

\subsection{General}

The first step in the analysis was to convert the logged target hit times into time differences between successive targets. Appendix E lists the resultant time differences. The second step was normalize the raw data from Appendix E by removing the effects properly attributed to variations in the level of course difficulty related to the Jason vehicle simulation model. This procedure divided each time difference (in seconds) by the best time (in seconds) that could be made between the associated targets based on the vehicle simulation. Appendix D discusses the method of arriving at this best time between targets and lists those times in table form. Appendix F contains the normalized, dimensionless values. The third step was to estimate the operators' learning curves to standardize results between tests. These results were then analyzed directly to assess the effectiveness of the tested display enhancements.

\subsection{Operator Learning Curves}

Operator learning reflected a number of factors including increasing familiarity with test equipment, improving knowledge of test procedures, and gradual easing of pretest anxiety, as well as modified strategies for driving the simulated vehicle. Estimation of the 
operators' learning curves involved four assumptions.

- The learning curves had positive slope at all times. This meant that the operators were constantly improving with no loss of knowledge or acquired skills between testing sessions.

- The learning curves were smooth functions. This prohibited the consideration of learning plateaus during which operators would show no improvement.

- The learning curves were exponential functions.

- For a specified field of view, operator learning was reflected primarily in the standard deviation of the normalized course times (a smoothness measure). The effectiveness of display enhancements was reflected primarily in the total course times (a measure of speed).

As an additional consideration, test 1 was designed to force the operators to use a variety of strategies, particularly with the reduced fields of view, in an attempt to ensure maximum learning in minimum time.

Each operator drove 21 courses with a $60^{\circ}$ field of view, three for each of the seven tests. The procedure below was applied to the normalized data for each operator to estimate his $60^{\circ}$ FOV learning curve.

- The standard deviations were entered into a $3 \times 7$ matrix. Each row represented a specific window width $\beta$ as discussed in section 5.2 (row $1=15^{\circ}$, row 2 $=30^{\circ}$, and row $3=60^{\circ}$ ). Each column corresponded to a given test (column $1=$ test 1 , column $2=$ test 2 , etc.).

- The standard deviations for the three window widths for each test were summed and entered into a row vector of seven elements. Each column continued to represent a given test as above. This step yielded the summed standard deviations (SSD) shown in table 6.1.

- The row vector elements were plotted with summed standard deviations on the vertical axis versus test number on the horizontal axis. 
- An exponential curve using the method of least squares was fitted to the plot by linearizing the exponential form through taking logarithms [G-1]. This defined the operator's learning curve. Appendix $\mathrm{G}$ shows the graphed operators' learning curves.

Once the operators' learning curves were estimated, correction factors to allow results between tests to be compared were derived using the following procedure.

- Estimated standard deviation values were extracted from the fitted exponential curve using regression techniques. This step produced the extracted values (EV) listed in table 6.1.

- Standardization factors (SF) were formed by dividing all EVs by the first (and largest) value. The EVs are listed in table 6.1.

- The 84 mean values of the normalized $60^{\circ}$ FOV course times listed in Appendix F were multiplied by the associated SF. This step resulted in the learningcorrected, normalized mean times (LCNMT) listed in table 6.2.

- For each test, the LCNMTs for all operators were summed. The mean and standard deviation of each LCNMT sum were found, completing the data manipulation.

Figure 6.1 shows the plotted means and standard deviations of the normalized mean times without correction for individual operator learning curves. Figure 6.2 shows the means and standard deviations of the LCNMT sum plotted for analysis.

The learning curve for the different fields of view could not be estimated using the same techniques. It was assumed that the individual operator's $60^{\circ} \mathrm{FOV}$ learning curve was a good approximation and the first three SFs from table 6.1 for each operator were applied to the data. 
Table 6.1 Summed standard deviations (SSD), extracted values (EV), and standardization factors (SF)

\begin{tabular}{|c|c|c|c|c|c|c|c|c|c|c|c|c|}
\cline { 2 - 13 } \multicolumn{1}{c|}{} & \multicolumn{4}{c|}{ Operator I } & \multicolumn{3}{c|}{ Operator II } & \multicolumn{3}{c|}{ Operator III } & \multicolumn{3}{c|}{ Operator IV } \\
\hline Test & SSD & EV & SF & SSD & EV & SF & SSD & EV & SF & SSD & EV & SF \\
\hline 1 & 4.16 & 3.56 & 1.00 & 7.65 & 5.86 & 1.00 & 7.35 & 5.32 & 1.00 & 3.85 & 3.51 & 1.00 \\
\hline 2 & 3.01 & 3.39 & 1.05 & 5.55 & 5.12 & 1.15 & 3.72 & 5.01 & 1.06 & 3.09 & 3.48 & 1.01 \\
\hline 3 & 3.36 & 3.22 & 1.11 & 4.41 & 4.47 & 1.31 & 5.70 & 4.71 & 1.13 & 3.48 & 3.46 & 1.01 \\
\hline 4 & 2.73 & 3.06 & 1.17 & 2.36 & 3.90 & 1.50 & 3.47 & 4.43 & 1.20 & 3.20 & 3.43 & 1.02 \\
\hline 5 & 2.75 & 2.91 & 1.23 & 2.66 & 3.40 & 1.72 & 3.45 & 4.17 & 1.28 & 3.59 & 3.41 & 1.03 \\
\hline 6 & 2.53 & 2.76 & 1.29 & 3.31 & 2.97 & 1.97 & 3.82 & 3.92 & 1.36 & 3.67 & 3.38 & 1.04 \\
\hline 7 & 3.10 & 2.62 & 1.36 & 3.56 & 2.59 & 2.26 & 4.82 & 3.69 & 1.44 & 3.20 & 3.36 & 1.04 \\
\hline
\end{tabular}

Table 6.2 Learning-corrected, normalized mean times $\left(60^{\circ} \mathrm{FOV}\right)$

\begin{tabular}{|c|c|c|c|c|c|c|c|c|c|c|c|c|}
\cline { 2 - 13 } \multicolumn{1}{c|}{} & \multicolumn{3}{|c|}{ Operator I } & \multicolumn{3}{c|}{ Operator II } & \multicolumn{3}{c|}{ Operator III } & \multicolumn{3}{c|}{ Operator IV } \\
\hline Test & $15^{\circ} \beta$ & $30^{\circ} \beta$ & $60^{\circ} \beta$ & $15^{\circ} \beta$ & $30^{\circ} \beta$ & $60^{\circ} \beta$ & $15^{\circ} \beta$ & $30^{\circ} \beta$ & $60^{\circ} \beta$ & $15^{\circ} \beta$ & $30^{\circ} \beta$ & $60^{\circ} \beta$ \\
\hline 1 & 1.84 & 2.26 & 3.62 & 3.00 & 2.90 & 5.55 & 2.52 & 3.32 & 3.85 & 3.70 & 3.15 & 3.48 \\
\hline 2 & 2.18 & 2.38 & 3.65 & 2.10 & 3.47 & 4.72 & 2.91 & 2.32 & 3.77 & 2.18 & 1.93 & 3.03 \\
\hline 3 & 1.90 & 1.85 & 3.26 & 3.49 & 4.12 & 4.65 & 3.09 & 3.26 & 2.81 & 1.61 & 1.94 & 2.95 \\
\hline 4 & 1.71 & 1.94 & 3.09 & 2.31 & 2.68 & 4.57 & 2.74 & 2.20 & 4.20 & 1.98 & 1.81 & 3.11 \\
\hline 5 & 2.65 & 2.02 & 3.43 & 3.23 & 3.68 & 4.78 & 2.98 & 2.69 & 3.11 & 1.61 & 1.56 & 2.93 \\
\hline 6 & 1.81 & 2.40 & 3.61 & 3.94 & 3.67 & 7.77 & 2.12 & 4.02 & 4.68 & 1.66 & 2.40 & 3.14 \\
\hline 7 & 2.71 & 3.60 & 3.97 & 6.33 & 5.87 & 7.81 & 3.58 & 4.66 & 5.63 & 2.06 & 2.20 & 3.40 \\
\hline
\end{tabular}




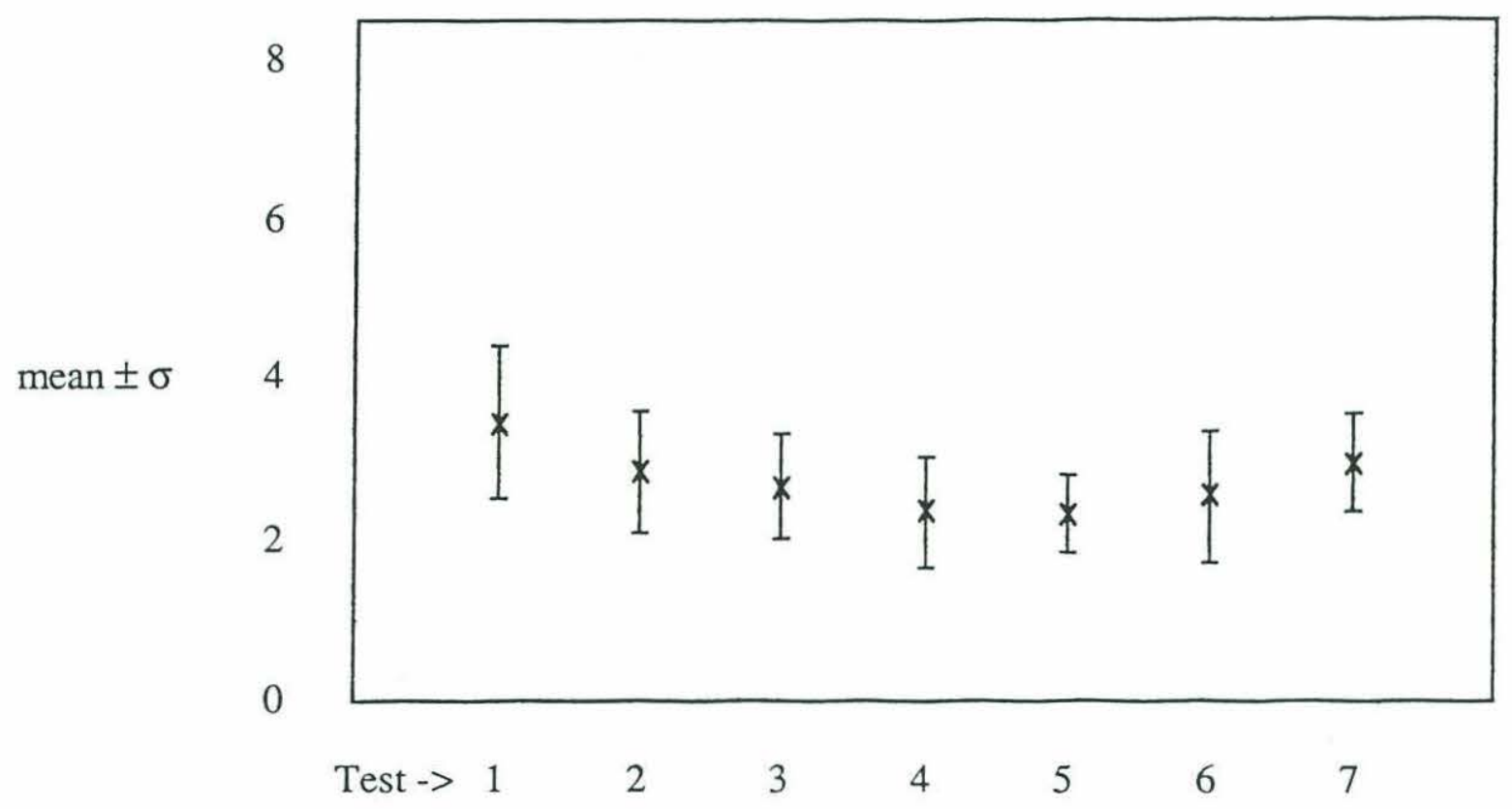

Figure $6.1 \quad$ Normalized mean times $\left(60^{\circ} \mathrm{FOV}\right)$

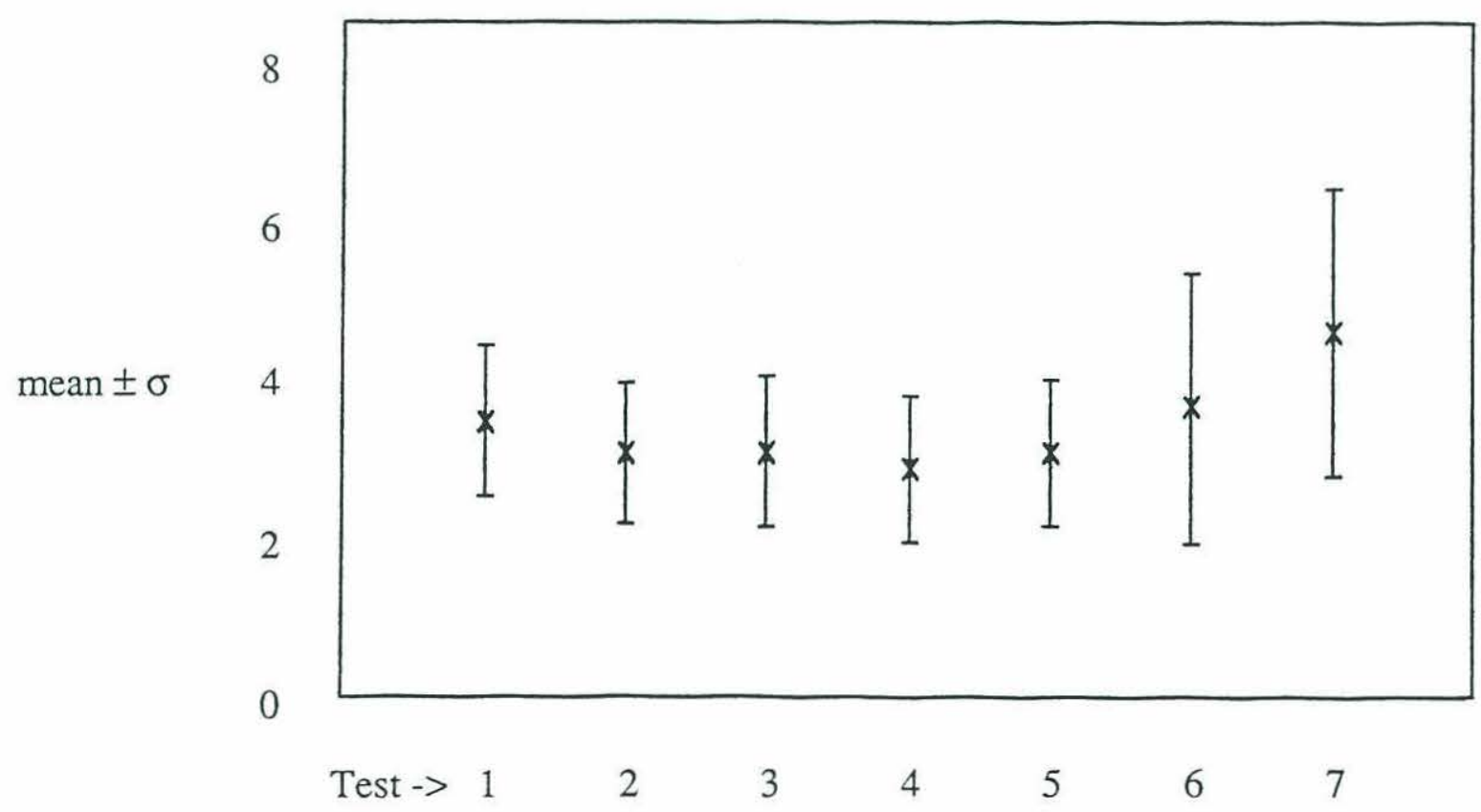

Figure 6.2 Learning-corrected, normalized mean times ( $\left.60^{\circ} \mathrm{FOV}\right)$ 


\subsection{Field of View}

The three fields of view used in testing were related by inverse powers of two $\left(60^{\circ}\right.$, $30^{\circ}$, and $15^{\circ}$ ). Plotted means and standard deviations of the learning-curve-corrected data versus FOV scaled by inverse powers of two were virtually linear and clearly demonstrated the parabolic relationship between the mean run time and the FOV. Fitting a parabola to the data showed that:

$$
\text { normalized mean run time }=10.399-0.200 * \mathrm{FOV}+1.350 * 10^{-3} * \mathrm{FOV}^{2}
$$

\subsection{Gridding}

The 2-D and 3-D grids improved operator performance to the same degree (34.64 and 34.92 respectively). The $2-\mathrm{D}$ grid was simple and straightforward to employ. The 3D grid conveyed more information, but cluttered the display and was harder to interpret. The primary benefit of the 2-D display was the "cross-hairs" effect of the grid, which aided steering effectiveness. This effect was less pronounced with the 3-D grid. Hidden-line removal, particularly for a 3-D grid, could provide strong visual range cues in the vicinity of a target.

\subsection{Vertical Color Schemes}

The 6-color scheme and the 21-color scheme both provided improved operator performance. The 6-color scheme proved to be slightly better (32.34 versus 34.68$)$. This effect was attributed to its simplicity and the high-contrast, easily distinguished colors. The 
Table 6.3 Learning-corrected, normalized mean times (varying FOV)

\begin{tabular}{|c|c|c|c|c|c|c|c|c|c|c|c|c|}
\cline { 2 - 13 } \multicolumn{1}{c|}{} & \multicolumn{3}{c|}{ Operator I } & \multicolumn{3}{c|}{ Operator II } & \multicolumn{3}{c|}{ Operator III } & \multicolumn{3}{c|}{ Operator IV } \\
\hline FOV & $15^{\circ} \beta$ & $30^{\circ} \beta$ & $60^{\circ} \beta$ & $15^{\circ} \beta$ & $30^{\circ} \beta$ & $60^{\circ} \beta$ & $15^{\circ} \beta$ & $30^{\circ} \beta$ & $60^{\circ} \beta$ & $15^{\circ} \beta$ & $30^{\circ} \beta$ & $60^{\circ} \beta$ \\
\hline 60 & 1.84 & 2.26 & 3.62 & 3.00 & 2.90 & 5.55 & 2.52 & 3.32 & 3.85 & 3.70 & 3.15 & 3.48 \\
\hline 30 & 5.89 & 5.07 & 4.99 & 4.63 & 4.73 & 6.05 & 6.49 & 5.71 & 7.13 & 3.80 & 4.11 & 8.83 \\
\hline 15 & 5.97 & 4.96 & 7.17 & 7.30 & 10.7 & 11.6 & 2.28 & 5.63 & 11.1 & 6.75 & 8.45 & 10.5 \\
\hline
\end{tabular}

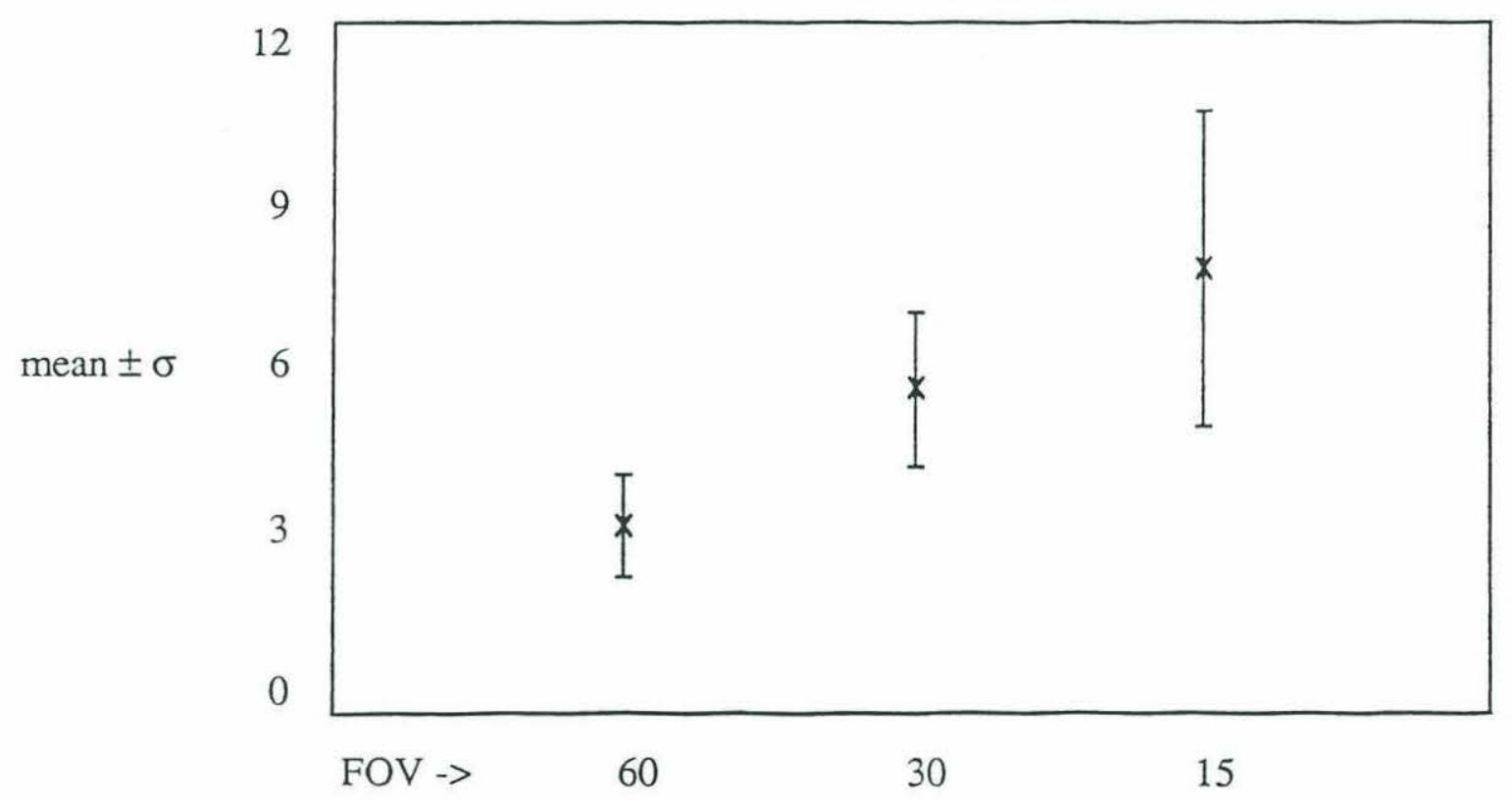

Figure 6.3 Learning-corrected, normalized mean times (varying FOV) 
primary benefit of the color schemes was improved spatial perception of the display due to the combined effects of depth and altitude cues. These two cues were strongly interrelated.

\subsection{Heads-Up Range}

A surprising result was the degradation of operator performance when supplied with range to the target center. Although the range information proved valuable if a target was not visible, was close, or had been missed, this was more than offset by negative factors. Primary problems included ignoring the visual information of the display and the "perigee effect," where a control action that caused the range to initially decrease would not necessarily result in a hit. As the vehicle's trajectory passed perigee with the target's center, the range increased, frequently confusing the operator. No individual operator's performance improved with range information.

\subsection{Display Update Rate}

As anticipated, slowing the graphics display update rate by a factor of two hurt operator performance. The magnitude of this effect, slowing the normalized run time by a factor of 1.3, was smaller than anticipated. This had significant implications for field applications, where a longer update period would allow the use of less powerful computer equipment or more robust programming. The loss of the operator's ability to mentally integrate the visual information from the display and use kinetic depth-effect cues was mitigated by the more conservative driving approach adopted by the operators. All the operators demonstrated this slowing effect with update rate. 


\subsection{Operator Comments}

Relevant operator comments were broken down into the categories of field of view, grids, color sets, range data, update rate, test equipment, test design, and strategy (operator number is included in parentheses after comments).

- Field of View- Narrowing the field of view placed a premium on maintaining visual contact with the targets (II).

- Grids - $\quad$ The "cross-hairs" effect aided steering effectiveness (II, IV).

Grids provided relative size information (II).

The 3-D grid was more intuitive and effective than the 2-D grid (II).

The 3-D grid cluttered the display (IV).

The grids were not particularly helpful (III).

Hidden-line removal for grids could provide strong visual range cues in the immediate vicinity of a target by showing the target "poking through" the grid (I, IV).

The black grids were sometimes confused with the blue targets when the display was cluttered (IV).

The operator's need for a grid was reduced by improvements in his spatial perception (IV).

- Color Sets - $\quad$ Color provided spatial visualization cues, especially with overlapping targets (all).

The 21-color set provided significantly more information and was more effective than the 6-color set (II, IV).

Color information was far superior to gridding (III). Target course altitude slope information was available from the rate of change of target colors (III). 
A sliding color scale with the vehicle always at scale center could provide improved relative altitude cues (III).

- Range Data - $\quad$ Range information proved valuable if a target was not visible, was close, or had been missed (all).

Range information was ignored if a target was in view (II).

Range information provided for smoother driving near targets and eased hitting targets off-center (II, IV).

The operator perceived that targets were "hit" from a greater distance with range information available (III).

- Update Rate - Operators drove more conservatively with the slower update rate (II, III).

Update rate was more important as the range decreased (III).

Vehicle alignment with the target trend was more important with the slower update rate due to the time lag in update rate while rotating (IV).

Operator workload increased with slower update rate (I).

The slow update rate was not as difficult as anticipated (IV).

- Test Equipment - The joystick was very tiring to use, particularly the z-axis pure-force control (III).

Fine $\mathrm{z}$-axis movement was difficult due to the stiff pureforce control (II, III).

Axis movement with the joystick was not compatible with operator expectations (I, III).

Presentation of all information on one screen could ease operator workload (all).

- Test Design - $\quad$ Target drawing order proved confusing (I, II, III). The display graphics can be cluttered, particularly with a 
narrow field of view (I, II, III).

The simulated vehicle lacked an effective altitude search capability (I, III).

Operator efficiency was reduced by the fixed 2-D target numbers when approaching targets from the side (all).

Improved heading perception was provided by the fixed 2-D target numbers (II).

- Strategy - Operator comfort with vehicle rotation improved as testing progressed (I, III).

Operators needed to plan more than one target in advance and, when possible, kept them on the display (II, III).

Rotating the vehicle to line up along the target heading trend reduced the time between targets (II, III).

Knowledge of the course trend aided target identification (IV).

Driving while backing up was counterintuitive (III).

\subsection{Test-Monitor Observations}

Test-monitor observations not previously listed under operator comments were broken down into the categories of color sets, range data, update rate, test equipment, test design, strategy, and tendencies.

- Color Sets - Some colors were weak in contrast with the background 
dark gray. Reddish Orange and Turquoise were most difficult for the operators to discern.

- Range Data - Range information sometimes handicapped the operators by providing too much data, causing them to ignore the display visual information. Another detrimental effect was that a control action that caused the range to initially decrease would not necessarily result in a hit. As the vehicle's trajectory passed perigee with the target's center, the range would increase. This effect fooled all operators initially, particularly when moving the vehicle along more than one axis simultaneously.

- Update Rate - The four-second update period significantly degraded the operator's ability to mentally integrate the display information and use kinetic depth-effect cues.

- Test Equipment - Stiffness of the joystick resulted in unorthodox grips being employed, many with both hands.

- Test Design - $\quad$ An artifact of the wireframe target design was that the simulated vehicle could be close to or within the target without any portion of the target being visible on the display. This is an unlikely scenario in the field.

- Strategy - $\quad$ Backing up to keep the previous target in view while looking for the next target was an effective approach when presented with a limited field of view.

Heading information was underutilized. Two possible uses were to measure the target trend heading and align 
the vehicle accordingly, and to improve the general sense of spatial orientation of the operator.

Operators adopted differing strategies for driving. These strategies were modified during testing but did not converge to a single "correct" method.

An effective search technique when no target was in view was critical in reducing course run time.

"Crabbing" or twisting one direction while moving side to side in the opposite direction was effective in realigning with a new course heading trend while keeping targets in view.

- Tendencies -

Applying excessive control actions and failing to account for the delay caused by the update rate was a problem, especially when in close proximity to a target.

Operators were hesitant to identify target numbers when only a portion of the number was visible or the angle of view approached $90^{\circ}$.

As testing progressed, the operators became more confident in controlling the vehicle in two or three directions simultaneously.

Operators occasionally applied the correct axis-control order in the opposite direction from that intended. This was related to the well-known outside-in versus inside-out viewing problem.

Operator average velocities increased as familiarity with the testing improved. 


\section{Chapter 7}

\section{Summary, Conclusions and Recommendations}

\subsection{Summary}

Effective collection, computation, and presentation of undersea data to the ROV pilot on a single screen presents hardware, software and human-factors problems. Pilot performance efficiency through improved display design is a key human factors issue. Seven tests measured the effects of an enhanced 3-D perspective display on the simulated piloting of an ROV. The effects of the ROV simulation and operator learning curves were removed to directly compare performance changes due to the various enhancements.

\subsection{Conclusions}

Display enhancements that improved operator performance were:

- widening the field of view.

- providing a gridded screen reference.

- using vertical color schemes to cue depth/altitude information.

- shortening the display update period.

The singularly most important factor for the operator was widening the field of view. Providing the operator with continuous range information to the target center 
degraded performance.

Many operator comments carried strong implications for system design.

- The "cross-hairs" effect of a grid aided steering effectiveness.

- Hidden-line removal for grids could provide strong visual-range cues in the immediate vicinity of a target by showing the target "poking through" the grid.

- Color provided spatial visualization cues and altitude slope information.

- Range information proved valuable if a target was not visible, was close, or had been missed.

- The update rate was more important as the range decreased.

- The joystick was very tiring to use. Fine z-axis movement was difficult due to the stiff pure-force control.

- Presentation of all information on one screen could ease operator workload.

Several operator comments were not supported by the test results.

- The 3-D grid was not more effective than the 2-D grid. They were nearly identical in overall effectiveness.

- The color schemes were not far superior to the grids. The difference between the 2-D grid, the 3-D grid, and the 21-color scheme was insignificant. The 6 color scheme was only slightly better.

- The 21-color set was not more effective than the 6-color set.

Several significant improvements to the proposed 3-D perspective display are available through incorporation of the best features of the display enhancements tested. A single screen with as wide a field of view as possible and limited distortion, a cross-hair, a simple vertical color scheme, target range information, and a reasonable update period (two 
seconds or less) would improve ROV pilot effectiveness. More testing would be necessary to determine the best design for a 2-D or 3-D grid. The key to effective use of range information is the method of employment.

\subsection{Recommendations}

This thesis investigated the effects of individual display enhancements on operator performance. In order to take maximum advantage of the positive aspects that each of the enhancement has to offer, several need to be implemented simultaneously. Due to the potentially complex nature of the enhancement interactions, it is unwise to generalize the individual results into group results. These interactions need to be analyzed on a case basis.

The presentation of both printed information such as position and velocities as well as graphics on the same screen was recommended by all operators. This capability exists in modern computer windowing environments. The effectiveness of any proposed screen layout design requires both qualitative and quantitative assessment.

One operator commented that a sliding color scale with the vehicle always at scale center could provide improved relative-altitude cues. This suggestion has merit and bears further investigation.

Both operators and pilots expressed strong dissatisfaction with the Measurement Systems, Inc., joystick designs (fourth-axis control by lifting/depressing the stick vertically or pure-force button). The sticks are far too difficult to use for extended periods due to their bulk, stiffness, and awkward incorporation of fourth-axis control. Catalog search has yet to produce a fully acceptable alternative, although a different joystick model is currently in use. Pilot preferences indicate that a modified video-game joystick is closer to their concept of an acceptable control. Identification of design requirements, development, 
testing to ensure effectiveness, and procurement of a fully acceptable control is an area needing attention.

Between testing sessions, usually a day or two apart, operators experienced a decay of previously acquired skills. The driving of course $\mathrm{X}$ prior to each test appeared to reverse this decay. ROV piloting is not a full-time occupation. Frequently months pass between cruises. Pilots also change employers, reducing the corporate piloting knowledge level. Three actions are recommended to reduce this loss of skills:

- Holding precruise pilot discussions to reawaken piloting skills. Critical to these discussions would be the minutes of the previous post-cruise debriefs. - Conduct of precruise pilot simulator training. Construction of a simulator with the field equipment would be straightforward and all the necessary hardware is already in hand. Pilot discussions of strategies could be demonstrated and tested prior to use in the field. Added benefits of a simulator include serving as a test bed for new display designs and the ability to write the simulator software to incorporate precruise system checks.

- Holding postcruise debriefs to document problems, fixes, and strategies. The minutes of these debriefs would generate a valuable storehouse of piloting knowledge. 


\section{APPENDIX A}

\section{Nomenclature}

\begin{tabular}{|c|c|c|c|}
\hline Symbol & Meaning & Units & Page \\
\hline $\mathrm{C}_{\mathrm{D}}$ & coefficient of drag & $\mathrm{kg} / \mathrm{m}$ or $\mathrm{kg}-\mathrm{m}$ & 38 \\
\hline $\mathrm{L}_{\mathrm{j}}$ & look direction matrix from point $\mathrm{P}_{\mathrm{j}}$ to & & \\
\hline \multirow{3}{*}{$\left(L_{j}\right)^{*}$} & point $\mathrm{P}_{\mathrm{j}+1}$ in vehicle system & none & 37 \\
\hline & transformation matrix from vehicle & & \\
\hline & system to world system & none & 37 \\
\hline M & vehicle mass & $\mathrm{kg}$ & 38 \\
\hline $\mathrm{M}_{\mathrm{a}}$ & vehicle added mass & $\mathrm{kg}$ & 38 \\
\hline $\mathrm{M}_{\kappa}$ & rotation matrix for $\kappa$ angular rotation & none & 37 \\
\hline $\mathrm{M}_{\varphi}$ & rotation matrix for $\varphi$ angular rotation & none & 37 \\
\hline $\mathrm{M}_{\omega}$ & rotation matrix for $\omega$ angular rotation & none & 37 \\
\hline$P_{j}$ & $\mathrm{j}$-th data point & none & 35 \\
\hline $\mathrm{T}$ & maximum thrust & $\mathrm{N}$ & 38 \\
\hline$\beta$ & operator field of view angular width & $\operatorname{rad}$ & 35 \\
\hline$\theta$ & vehicle system rotation angle about $\mathrm{z}$ axis & $\mathrm{rad}$ & 26 \\
\hline$\kappa$ & world system rotation angle about $\mathrm{z}$ axis & $\mathrm{rad}$ & 36 \\
\hline$\sigma$ & standard deviation & none & 51 \\
\hline$\tau_{\max }$ & linear drag (damping) coefficient & $\mathrm{S}$ & 38 \\
\hline$\varphi$ & world system rotation angle about y axis & $\mathrm{rad}$ & 36 \\
\hline$\omega$ & world system rotation angle about $\mathrm{x}$ axis & $\mathrm{rad}$ & 36 \\
\hline
\end{tabular}


kg kilograms

m meters

$\mathrm{N}$ newtons

rad radians

s seconds 


\section{APPENDIX B}

\section{Abbreviations}

Symbol Meaning Page

ACM Association for Computing Machinery 133

BBN Bolt, Beranek, and Newman 23

$\begin{array}{lll}\text { CCW } & \text { Counterclockwise } & 38\end{array}$

CDR Commander 3

CIE Commission Internationale de l'Eclairage 19

$\begin{array}{lll}\text { CRT } & \text { Cathode Ray Tube } & 13\end{array}$

CW Clockwise 38

DSL Deep Submergence Laboratory 8

$\begin{array}{ll}\text { DV Dependent Variable } & 22\end{array}$

$\begin{array}{lll}\text { EV Extracted Value } & 10\end{array}$

FJCC Fall Joint Computer Congress 134

FOV Field of View $\quad 8$

$\begin{array}{lll}\text { GDM } & \text { Graphic Display Monitor } & 27\end{array}$

HOOPS Hierarchical Object Oriented Picture System 25

HSB Hue, Saturation, and Brightness 10

IEEE Institute of Electrical and Electronics Engineers 133

IFIP International Federation for Information Processing 134

ISCC-NBS Inter-Society Color Council - National Bureau of Standards 19

IV Independent Variable $\quad 22$

$\begin{array}{lll}\text { LCDR } & 136\end{array}$ 
LCNMT Learning-Corrected, Normalized Mean Times $\quad 49$

MIPS Millions of Instructions Per Second 26

MIT Massachusetts Institute of Technology 1

MFLOPS Millions of Floating-Point Operations Per Second 26

$\begin{array}{lll}\text { N/A } & \text { Not Applicable } & 73\end{array}$

$\begin{array}{lll}\text { NEC Nippon Electric Company } & 27\end{array}$

NTSC National Television System Committee 26

$\begin{array}{lll}\text { RGB } & \text { Red, Green, Blue }\end{array}$

ROV Remotely Operated Vehicle 2

SF Standardization Factor $\quad 10$

SHARPS Sonic High Accuracy Ranging and Positioning System 31

SONAR Sound Navigation and Ranging 2

SSD Summed Standard Deviation $\quad 10$

$\begin{array}{lll}\text { TI Texas Instruments } & 23\end{array}$

UCL Universal Color Language 19

USN United States Navy 3

VCR Video Cassette Recorder 26

$\begin{array}{lll}\text { VGA } & \text { Video Graphics Adapter } & 27\end{array}$

WHOI Woods Hole Oceanographic Institution 1

YIQ Luminance, In-phase, and Quadrature 19

2-D Two-Dimensional $\quad 8$

3-D Three-Dimensional 2 


\section{APPENDIX C \\ Target and Grid Design}

All seven tests used the same 25 cubic-wireframe targets. Each target was one meter on a side with the invisible $\mathrm{x}, \mathrm{y}$, and $\mathrm{z}$ data point at its center. Target faces were parallel to the planes formed by the world coordinate system axes. Target colors were blue except for tests 5 and 6 as discussed in section 5.7. Each target was numbered with white numerals (1-25) corresponding to the sequential number of the data point at its center. The numbers were 2-D wireframes 0.5 meters in height, 0.3 meters in width (numeral one was 0.1 meters in width), and drawn parallel to the $\mathrm{XZ}$ world coordinate plane facing the initial position of the vehicle. All numbers were drawn centered in the target in the $\mathrm{x}, \mathrm{y}$, and $\mathrm{z}$ directions.

Embedded in each target was an invisible sphere of one-meter diameter. This sphere defined the "hit" criterion for its target. When the vehicle, modeled as a point, touched the sphere, the target was hit. This corresponded to closing the range to the target center to 0.5 meters or less from any direction.

The 2-D grid used in test 2 was composed of 16 black squares arranged in four rows of four. The grid was drawn centered, parallel to, and aligned with the edges of the display face at a screen depth of five meters. Each square was scaled so it was one meter on a side at that depth. Figure C.1 shows the operator's display of the 2-D grid with target course X.

The 3-D grid used in test 3 was composed of 200 black squares and rectangles arranged in three panels. The center panel was made up of 100 squares in 10 rows of 10. This grid was drawn centered, parallel to, and aligned with the edges of the display face at a screen depth of five meters. Each square was scaled so that it was 0.25 meter on a side at 
that depth. The side panels were each made up of 50 rectangles in 10 rows of five. Each rectangle was 0.25 meter in height and one meter in width. The side panels were attached to the center grid at right angles to its left and right edges. The assembled grid formed a box with no top or bottom facing the operator. Figure C. 2 shows the operator's display of the 3-D grid with target course X. 


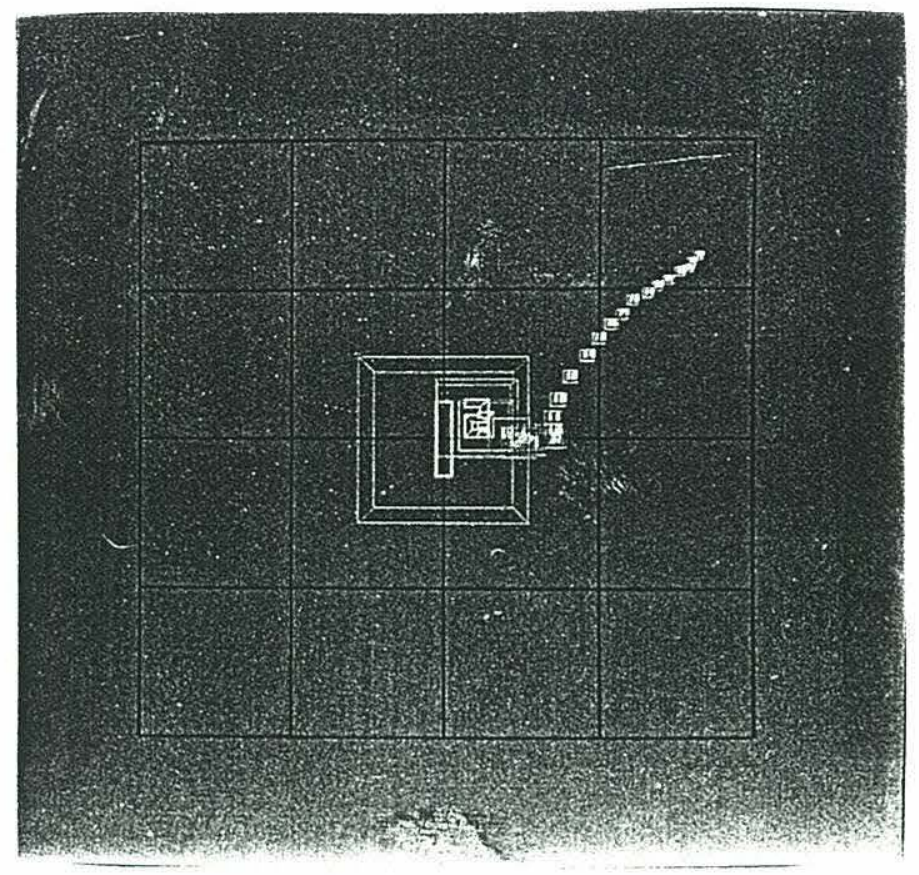

Figure C.1 2-D grid (operator's display with $60^{\circ}$ FOV and target course X)

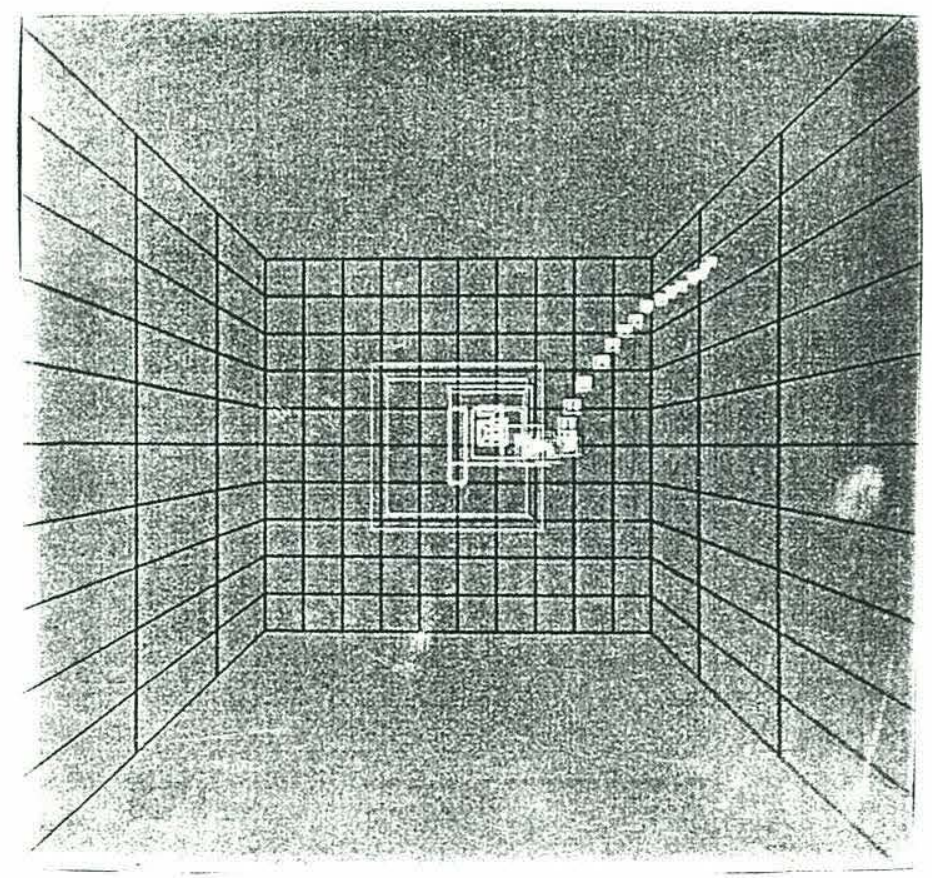

Figure C.2 3-D grid (operator's display with $60^{\circ}$ FOV and target course $\mathrm{X}$ ) 


\section{APPENDIX D}

\section{Data Point Courses}

Tables D.1 through D.19 list the $\mathrm{x}, \mathrm{y}$, and $\mathrm{z}$ positions of the centers of targets 1 through 25 for each of the target courses. The method for generating these $\mathrm{x}, \mathrm{y}$, and $\mathrm{z}$ positions is discussed in detail in section 5.2. Additionally, the shortest time difference (best $\delta \mathrm{t}$ ) between the centers of successive targets is listed for courses A through $\mathrm{R}$. The best $\delta \mathrm{t}$ between targets $\mathrm{U}$ and $\mathrm{V}$ was calculated as follows:

- altitude distance from $\mathrm{z}_{\mathrm{u}}$ to $\mathrm{z}_{\mathrm{v}}$ was calculated, then divided by the best up/down speed $(0.25 \mathrm{~m} / \mathrm{s})$, resulting in the best up/down time.

- the change in vehicle heading at target $\mathrm{U}$ to fly directly to target $\mathrm{V}$ was noted.

- distance in the horizontal plane from $\left(\mathrm{x}_{\mathrm{u}}, \mathrm{y}_{\mathrm{u}}\right)$ to $\left(\mathrm{x}_{\mathrm{V}}, \mathrm{y}_{\mathrm{V}}\right)$ was calculated.

- this horizontal distance was decomposed into forward and lateral components based on the vehicle's heading prior to reaching target $\mathrm{U}$.

- the decomposed forward distance was divided by the best forward speed $(0.5$ $\mathrm{m} / \mathrm{s})$.

- the decomposed lateral distance was divided by the best lateral speed $(0.25 \mathrm{~m} / \mathrm{s})$.

- the minimum time to travel the horizontal distance without heading change was the larger of the best forward and lateral speed times.

- vehicle heading change was divided by the best rotational velocity $(0.157 \mathrm{rad} / \mathrm{s})$.

- the total horizontal distance was divided by the best forward speed $(0.5 \mathrm{~m} / \mathrm{s})$.

- the minimum time to travel the horizontal distance with heading change was the sum of the best rotational velocity time and the best forward speed time.

- the minimum time to travel the horizontal distance was the smaller of the times with and without heading change. 
- the best time between targets was the larger of the best up/down and horizontal times.

Figures D.1 through D.18 were reproduced from photographs of the operator's initial display for courses A through $\mathrm{R}$ with a $60^{\circ}$ field of view. Figures D.19, D.20, and D.21 were reproductions of the initial display for course $\mathrm{X}$ with a $60^{\circ}, 30^{\circ}$, and $15^{\circ}$ field of view respectively. 
Table D.1 Course A

\begin{tabular}{|c|c|c|c|c|}
\hline Target & $\mathrm{x}$ & $\mathrm{y}$ & z & Best $\delta t$ \\
\hline 1 & 75 & 5 & 50 & N/A \\
\hline 2 & 75.340 & 9.971 & 50.419 & 9.942 \\
\hline 3 & 75.683 & 14.959 & 50.393 & 10.000 \\
\hline 4 & 75.743 & 19.937 & 50.862 & 9.940 \\
\hline 5 & 76.337 & 24.897 & 51.060 & 9.935 \\
\hline 6 & 77.204 & 29.809 & 50.703 & 9.959 \\
\hline 7 & 78.401 & 34.662 & 50.821 & 9.975 \\
\hline 8 & 79.848 & 39.447 & 50.718 & 9.984 \\
\hline 9 & 80.992 & 44.314 & 50.665 & 9.980 \\
\hline 10 & 82.121 & 49.184 & 50.707 & 10.000 \\
\hline 11 & 83.810 & 53.888 & 50.570 & 9.928 \\
\hline 12 & 86.077 & 58.330 & 50.930 & 9.894 \\
\hline 13 & 88.094 & 62.885 & 51.366 & 9.947 \\
\hline 14 & 90.093 & 67.411 & 52.085 & 9.896 \\
\hline 15 & 91.666 & 72.019 & 53.219 & 9.704 \\
\hline 16 & 92.761 & 76.807 & 54.158 & 9.770 \\
\hline 17 & 93.676 & 81.488 & 55.659 & 9.536 \\
\hline 18 & 94.076 & 86.374 & 56.640 & 9.742 \\
\hline 19 & 94.950 & 91.283 & 57.016 & 9.935 \\
\hline 20 & 95.347 & 96.261 & 57.262 & 9.940 \\
\hline 21 & 95.595 & 101.250 & 57.047 & 9.984 \\
\hline 22 & 95.454 & 106.237 & 56.713 & 9.947 \\
\hline 23 & 95.215 & 111.159 & 55.866 & 9.852 \\
\hline 24 & 94.726 & 115.948 & 54.514 & 9.612 \\
\hline 25 & 94.792 & 120.760 & 53.159 & 9.566 \\
\hline
\end{tabular}


Table D.2 Course B

\begin{tabular}{|c|c|c|c|c|}
\hline Target & $\mathrm{x}$ & $\mathrm{y}$ & $\mathrm{z}$ & Best $\delta \mathrm{t}$ \\
\hline 1 & 75 & 5 & 50 & N/A \\
\hline 2 & 75.514 & 9.942 & 50.561 & 9.884 \\
\hline 3 & 76.280 & 14.801 & 51.459 & 9.825 \\
\hline 4 & 76.464 & 19.731 & 52.270 & 9.800 \\
\hline 5 & 76.788 & 24.651 & 53.100 & 9.857 \\
\hline 6 & 77.619 & 29.517 & 53.893 & 9.822 \\
\hline 7 & 78.714 & 34.369 & 54.406 & 9.933 \\
\hline 8 & 80.191 & 39.111 & 54.981 & 9.903 \\
\hline 9 & 82.247 & 43.558 & 55.978 & 9.723 \\
\hline 10 & 83.945 & 48.008 & 57.501 & 9.500 \\
\hline 11 & 85.987 & 52.139 & 59.440 & 9.186 \\
\hline 12 & 88.368 & 56.088 & 61.373 & 9.196 \\
\hline 13 & 90.182 & 60.266 & 63.436 & 9.039 \\
\hline 14 & 91.667 & 64.362 & 65.889 & 9.812 \\
\hline 15 & 92.799 & 68.246 & 68.827 & 11.755 \\
\hline 16 & 93.692 & 72.084 & 71.905 & 12.311 \\
\hline 17 & 94.672 & 75.833 & 75.064 & 12.636 \\
\hline 18 & 96.247 & 79.760 & 77.729 & 10.659 \\
\hline 19 & 97.585 & 83.600 & 80.639 & 11.639 \\
\hline 20 & 98.424 & 87.377 & 83.806 & 12.669 \\
\hline 21 & 99.684 & 90.714 & 87.309 & 14.012 \\
\hline 22 & 101.492 & 93.759 & 90.839 & 14.118 \\
\hline 23 & 102.575 & 96.682 & 94.748 & 15.637 \\
\hline 24 & 103.974 & 99.466 & 98.659 & 15.643 \\
\hline 25 & 105.626 & 101.705 & 102.813 & 16.618 \\
\hline
\end{tabular}




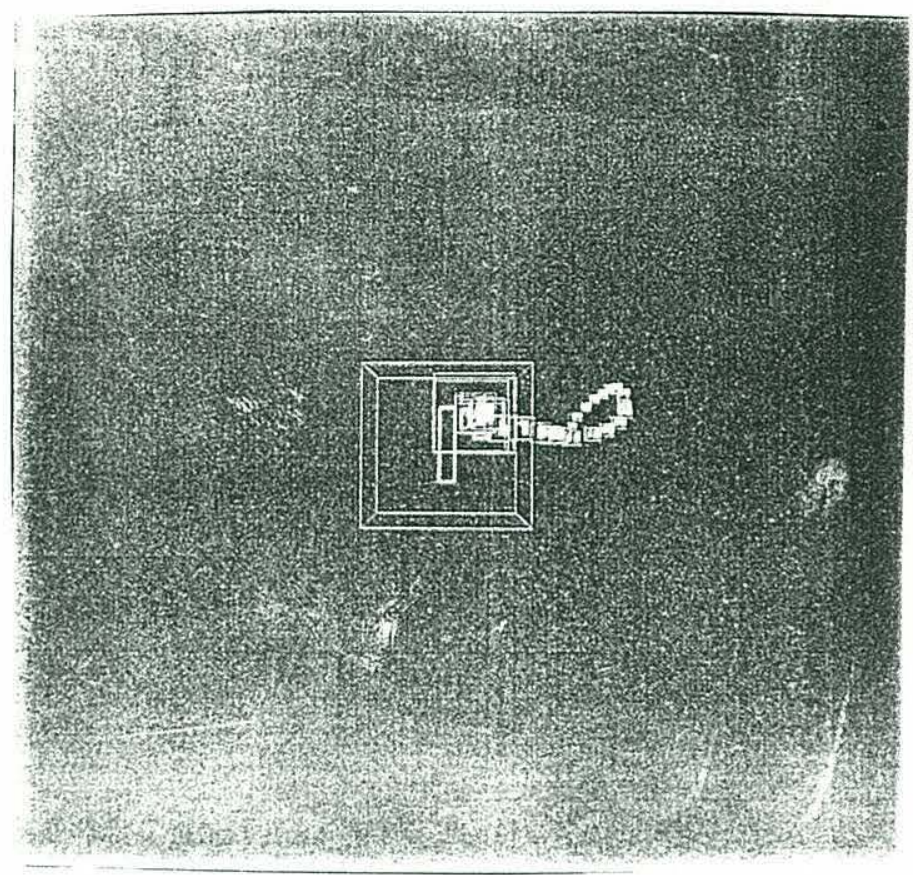

Figure D.1 Course A (operator's display with $60^{\circ}$ FOV)

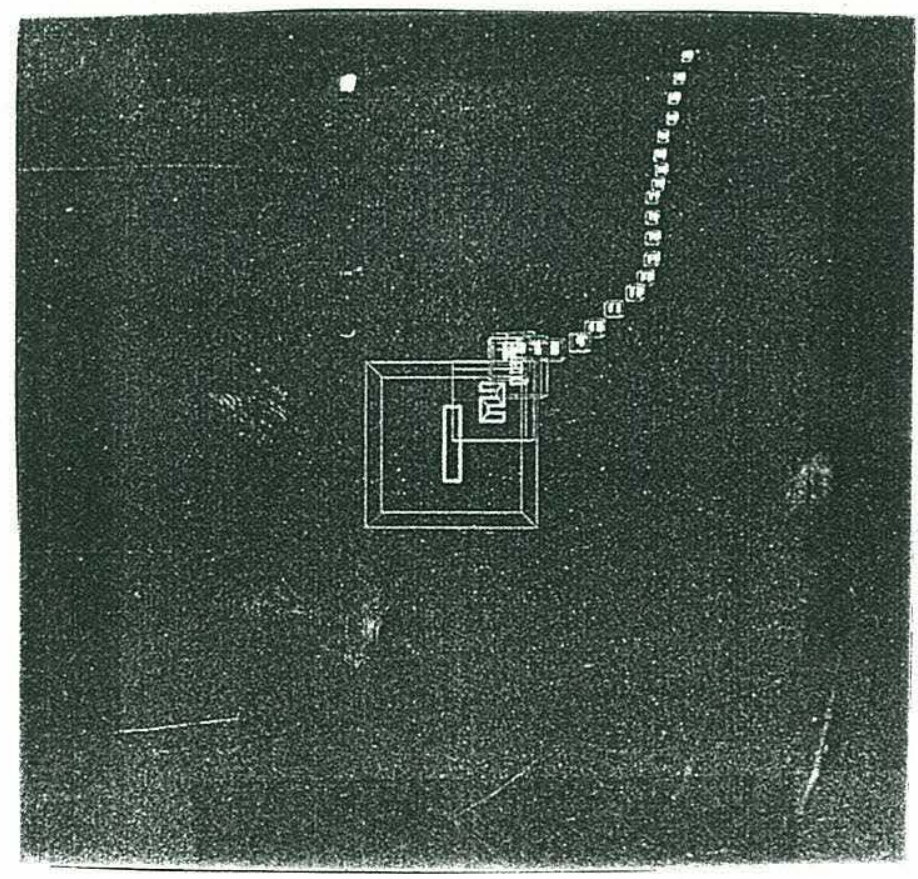

Figure D.2 Course B (operator's display with $60^{\circ} \mathrm{FOV}$ ) 
Table D.3 Course C

\begin{tabular}{|c|c|c|c|c|}
\hline Target & $\mathrm{x}$ & $\mathrm{y}$ & $\mathrm{z}$ & Best $\delta \mathrm{t}$ \\
\hline 1 & 75 & 5 & 50 & N/A \\
\hline 2 & 75.020 & 9.983 & 50.415 & 9.965 \\
\hline 3 & 74.980 & 14.977 & 50.180 & 9.988 \\
\hline 4 & 74.925 & 19.976 & 50.095 & 9.999 \\
\hline 5 & 75.269 & 24.919 & 49.429 & 9.879 \\
\hline 6 & 75.592 & 29.763 & 48.232 & 9.709 \\
\hline 7 & 76.443 & 34.610 & 47.343 & 9.786 \\
\hline 8 & 77.675 & 39.396 & 46.586 & 9.855 \\
\hline 9 & 78.482 & 44.192 & 45.423 & 9.690 \\
\hline 10 & 79.342 & 48.955 & 44.170 & 9.680 \\
\hline 11 & 80.071 & 53.561 & 42.368 & 9.325 \\
\hline 12 & 80.670 & 58.230 & 40.681 & 9.410 \\
\hline 13 & 81.492 & 63.000 & 39.427 & 9.672 \\
\hline 14 & 82.575 & 67.842 & 38.810 & 9.911 \\
\hline 15 & 83.997 & 72.636 & 38.769 & 9.974 \\
\hline 16 & 85.192 & 77.489 & 38.898 & 9.986 \\
\hline 17 & 86.296 & 82.364 & 39.015 & 9.995 \\
\hline 18 & 87.120 & 87.270 & 38.511 & 9.932 \\
\hline 19 & 88.257 & 92.015 & 37.420 & 9.738 \\
\hline 20 & 89.117 & 96.742 & 36.035 & 9.594 \\
\hline 21 & 89.412 & 101.410 & 34.268 & 9.297 \\
\hline 22 & 89.760 & 106.245 & 33.043 & 9.695 \\
\hline 23 & 89.707 & 111.017 & 31.552 & 9.516 \\
\hline 24 & 89.728 & 115.746 & 29.930 & 9.458 \\
\hline 25 & 89.851 & 120.359 & 28.004 & 9.226 \\
\hline & & & & \\
\hline
\end{tabular}


Table D.4 Course D

\begin{tabular}{|c|c|c|c|c|}
\hline Target & $\mathrm{x}$ & $\mathrm{y}$ & $\mathrm{z}$ & Best $\delta \mathrm{t}$ \\
\hline 1 & 75 & 5 & 50 & N/A \\
\hline 2 & 74.372 & 9.917 & 50.658 & 9.835 \\
\hline 3 & 74.614 & 14.903 & 50.928 & 9.835 \\
\hline 4 & 74.826 & 19.671 & 52.420 & 9.544 \\
\hline 5 & 75.283 & 24.294 & 54.270 & 9.278 \\
\hline 6 & 76.893 & 28.957 & 55.080 & 9.601 \\
\hline 7 & 79.632 & 33.111 & 55.573 & 9.637 \\
\hline 8 & 82.281 & 37.123 & 56.946 & 9.615 \\
\hline 9 & 85.192 & 41.067 & 57.934 & 9.787 \\
\hline 10 & 87.250 & 45.189 & 59.876 & 9.073 \\
\hline 11 & 89.045 & 48.851 & 62.768 & 11.570 \\
\hline 12 & 91.190 & 52.496 & 65.436 & 10.670 \\
\hline 13 & 93.106 & 56.769 & 67.187 & 9.327 \\
\hline 14 & 94.735 & 61.062 & 69.167 & 9.169 \\
\hline 15 & 95.491 & 65.646 & 71.015 & 9.134 \\
\hline 16 & 95.769 & 70.151 & 73.167 & 8.993 \\
\hline 17 & 95.051 & 74.059 & 76.202 & 12.139 \\
\hline 18 & 94.195 & 78.131 & 78.975 & 11.092 \\
\hline 19 & 94.587 & 82.593 & 81.196 & 8.885 \\
\hline 20 & 95.963 & 86.945 & 83.236 & 8.962 \\
\hline 21 & 96.694 & 91.569 & 84.993 & 9.265 \\
\hline 22 & 98.573 & 96.153 & 85.668 & 9.709 \\
\hline 23 & 100.225 & 100.734 & 86.802 & 9.734 \\
\hline 24 & 101.228 & 105.627 & 86.583 & 9.845 \\
\hline 25 & 101.964 & 110.571 & 86.428 & 9.981 \\
\hline
\end{tabular}




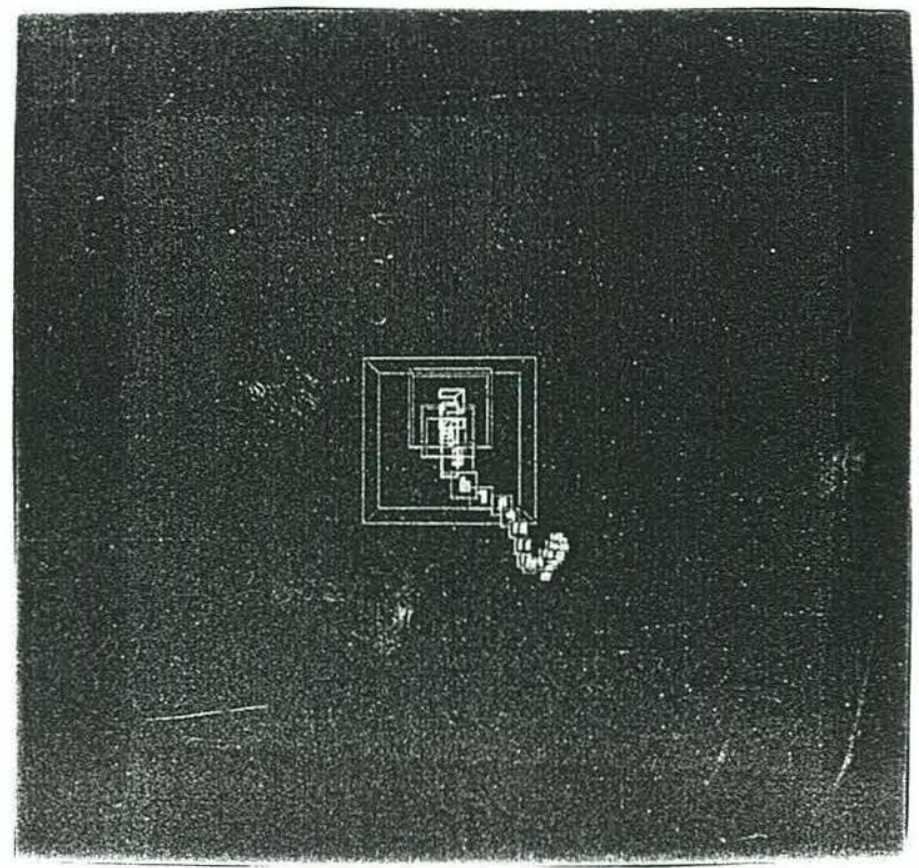

Figure D. 3 Course C (operator's display with $60^{\circ} \mathrm{FOV}$ )

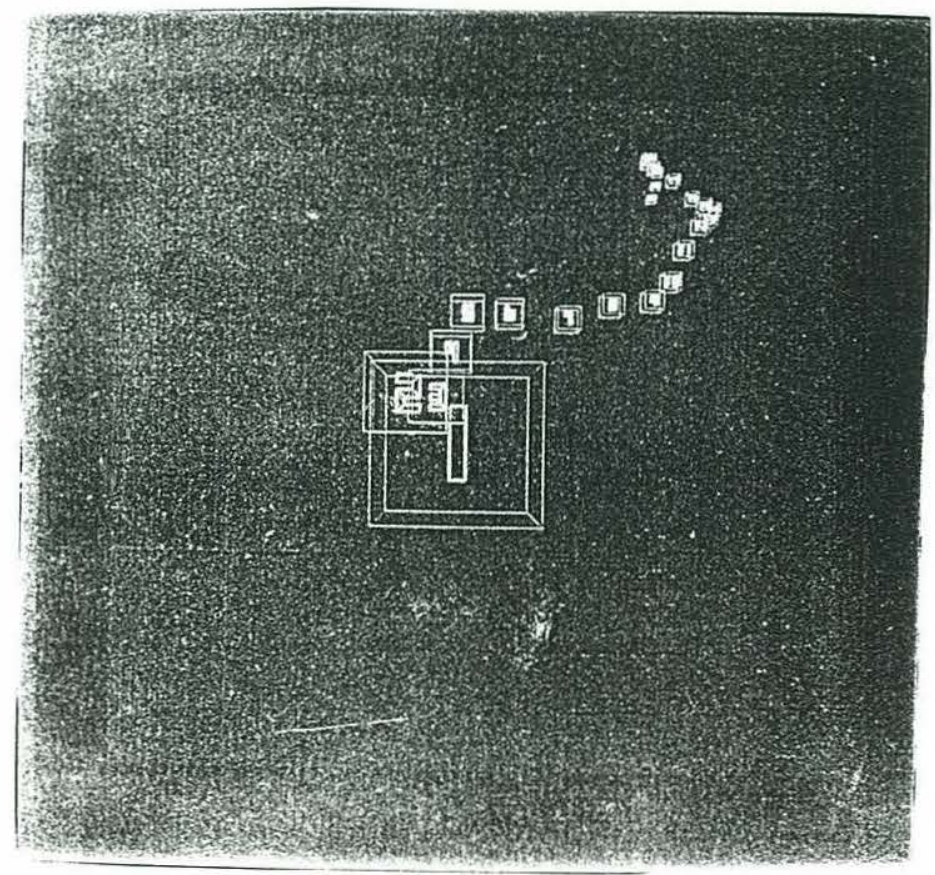

Figure D.4 Course D (operator's display with $60^{\circ} \mathrm{FOV}$ ) 
Table D.5 Course E

\begin{tabular}{|c|c|c|c|c|}
\hline Target & $\mathrm{x}$ & $y$ & $\mathrm{z}$ & Best $\delta \mathrm{t}$ \\
\hline 1 & 75 & 5 & 50 & N/A \\
\hline 2 & 74.164 & 9.874 & 50.737 & 9.752 \\
\hline 3 & 72.990 & 14.566 & 52.005 & 9.649 \\
\hline 4 & 72.104 & 19.139 & 53.823 & 9.301 \\
\hline 5 & 70.407 & 23.580 & 55.370 & 9.378 \\
\hline 6 & 68.214 & 28.060 & 55.718 & 9.917 \\
\hline 7 & 66.758 & 32.567 & 57.322 & 9.357 \\
\hline 8 & 64.791 & 36.764 & 59.197 & 9.209 \\
\hline 9 & 63.625 & 41.499 & 60.302 & 9.599 \\
\hline 10 & 61.257 & 45.902 & 60.362 & 9.663 \\
\hline 11 & 59.506 & 50.584 & 60.486 & 9.905 \\
\hline 12 & 58.125 & 55.374 & 60.874 & 9.939 \\
\hline 13 & 57.941 & 60.311 & 61.644 & 9.591 \\
\hline 14 & 58.232 & 65.298 & 61.853 & 9.945 \\
\hline 15 & 59.369 & 70.150 & 62.256 & 9.822 \\
\hline 16 & 60.703 & 74.966 & 62.424 & 9.986 \\
\hline 17 & 63.211 & 79.278 & 62.770 & 9.653 \\
\hline 18 & 65.725 & 83.598 & 62.902 & 9.997 \\
\hline 19 & 68.894 & 87.449 & 63.255 & 9.849 \\
\hline 20 & 71.096 & 91.933 & 63.470 & 9.728 \\
\hline 21 & 73.080 & 96.326 & 64.797 & 9.633 \\
\hline 22 & 75.684 & 100.527 & 65.557 & 9.796 \\
\hline 23 & 77.857 & 105.028 & 65.683 & 9.949 \\
\hline 24 & 79.568 & 109.626 & 64.715 & 9.780 \\
\hline 25 & 81.847 & 113.963 & 63.718 & 9.723 \\
\hline
\end{tabular}


Table D.6 Course F

\begin{tabular}{|c|c|c|c|c|}
\hline Target & $\mathrm{x}$ & $\mathrm{y}$ & $\mathrm{z}$ & Best $\delta \mathrm{t}$ \\
\hline 1 & 75 & 5 & 50 & N/A \\
\hline 2 & 74.090 & 9.822 & 50.957 & 9.651 \\
\hline 3 & 72.789 & 14.460 & 52.299 & 9.602 \\
\hline 4 & 71.500 & 18.826 & 54.367 & 9.105 \\
\hline 5 & 71.318 & 23.509 & 56.109 & 9.130 \\
\hline 6 & 71.669 & 28.250 & 57.657 & 9.453 \\
\hline 7 & 70.716 & 32.909 & 59.205 & 9.185 \\
\hline 8 & 69.282 & 37.669 & 59.737 & 9.897 \\
\hline 9 & 67.473 & 42.322 & 60.019 & 9.952 \\
\hline 10 & 65.537 & 46.929 & 60.170 & 9.991 \\
\hline 11 & 63.072 & 51.277 & 60.311 & 9.927 \\
\hline 12 & 61.000 & 55.811 & 59.923 & 9.936 \\
\hline 13 & 58.565 & 60.063 & 58.930 & 9.756 \\
\hline 14 & 56.794 & 64.739 & 58.944 & 9.864 \\
\hline 15 & 53.946 & 68.755 & 58.072 & 9.523 \\
\hline 16 & 51.351 & 73.023 & 57.847 & 9.966 \\
\hline 17 & 49.014 & 77.376 & 57.080 & 9.867 \\
\hline 18 & 45.742 & 80.745 & 55.364 & 9.074 \\
\hline 19 & 41.720 & 83.431 & 54.096 & 9.457 \\
\hline 20 & 38.238 & 87.010 & 53.838 & 9.831 \\
\hline 21 & 34.887 & 90.688 & 54.334 & 9.950 \\
\hline 22 & 31.512 & 94.286 & 53.521 & 9.864 \\
\hline 23 & 29.020 & 98.614 & 53.754 & 9.792 \\
\hline 24 & 27.165 & 103.247 & 54.071 & 9.886 \\
\hline 25 & 25.496 & 107.871 & 53.158 & 9.806 \\
\hline & & & & \\
\hline
\end{tabular}




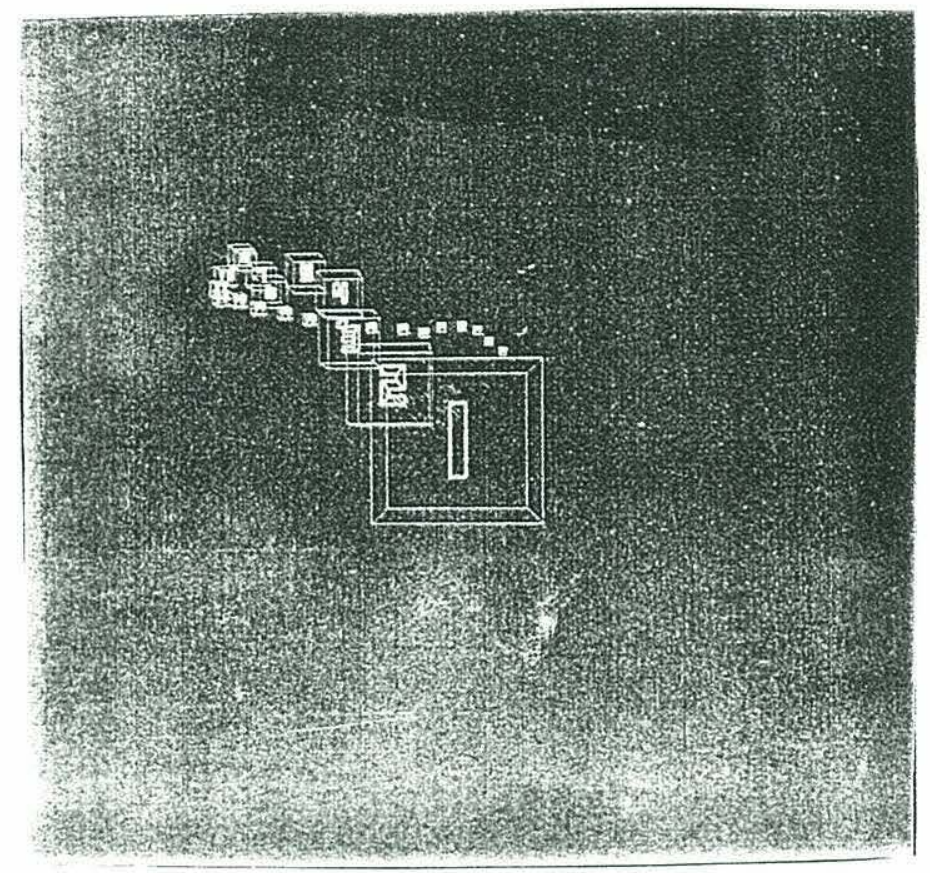

Figure D.5 Course E (operator's display with $60^{\circ}$ FOV)

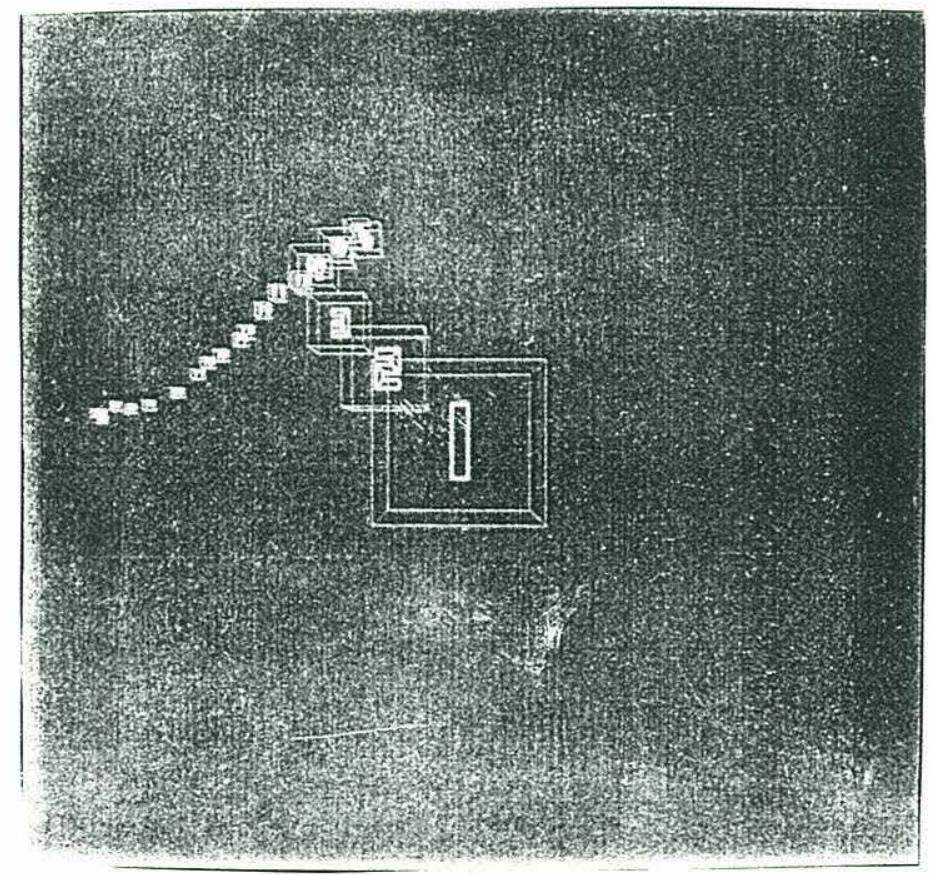

Figure D.6 Course F (operator's display with $60^{\circ}$ FOV) 
Table D.7 Course G

\begin{tabular}{|c|c|c|c|c|}
\hline Target & $\mathrm{x}$ & $\mathrm{y}$ & $\mathrm{z}$ & Best $\delta \mathrm{t}$ \\
\hline 1 & 75 & 5 & 50 & $\mathrm{~N} / \mathrm{A}$ \\
\hline 2 & 77.402 & 9.372 & 49.658 & 9.584 \\
\hline 3 & 80.930 & 12.001 & 47.283 & 9.501 \\
\hline 4 & 85.722 & 13.316 & 46.731 & 8.949 \\
\hline 5 & 90.100 & 13.069 & 44.328 & 9.609 \\
\hline 6 & 95.045 & 13.750 & 44.040 & 9.983 \\
\hline 7 & 99.803 & 12.432 & 43.248 & 9.344 \\
\hline 8 & 104.761 & 12.926 & 42.829 & 9.532 \\
\hline 9 & 109.181 & 12.034 & 44.988 & 8.637 \\
\hline 10 & 111.078 & 10.074 & 49.179 & 16.763 \\
\hline 11 & 110.555 & 7.725 & 53.562 & 17.531 \\
\hline 12 & 110.822 & 7.113 & 58.517 & 19.820 \\
\hline 13 & 112.792 & 8.030 & 63.020 & 18.011 \\
\hline 14 & 115.272 & 10.445 & 66.628 & 14.434 \\
\hline 15 & 116.622 & 14.175 & 69.671 & 12.174 \\
\hline 16 & 118.163 & 16.656 & 73.730 & 16.233 \\
\hline 17 & 117.290 & 18.362 & 78.348 & 18.473 \\
\hline 18 & 118.453 & 19.258 & 83.127 & 19.118 \\
\hline 19 & 120.460 & 18.207 & 87.585 & 17.829 \\
\hline 20 & 123.046 & 15.938 & 91.212 & 14.510 \\
\hline 21 & 126.242 & 14.134 & 94.609 & 13.584 \\
\hline 22 & 130.759 & 13.240 & 96.559 & 8.860 \\
\hline 23 & 135.067 & 14.923 & 98.456 & 9.114 \\
\hline 24 & 139.759 & 16.585 & 98.932 & 9.935 \\
\hline 25 & 144.021 & 18.058 & 101.093 & 8.962 \\
\hline
\end{tabular}


Table D. 8 Course H

\begin{tabular}{|c|c|c|c|c|}
\hline Target & $\mathrm{x}$ & $\mathrm{y}$ & $\mathrm{z}$ & Best $\delta \mathrm{t}$ \\
\hline 1 & 75 & 5 & 50 & N/A \\
\hline 2 & 73.035 & 9.488 & 50.999 & 9.010 \\
\hline 3 & 71.086 & 14.085 & 51.257 & 9.987 \\
\hline 4 & 69.943 & 18.154 & 53.928 & 10.684 \\
\hline 5 & 68.558 & 21.353 & 57.512 & 14.338 \\
\hline 6 & 65.215 & 23.831 & 60.286 & 11.093 \\
\hline 7 & 60.466 & 25.325 & 60.742 & 9.566 \\
\hline 8 & 55.907 & 26.833 & 62.137 & 9.558 \\
\hline 9 & 52.630 & 30.596 & 62.454 & 9.227 \\
\hline 10 & 52.017 & 35.556 & 62.305 & 9.426 \\
\hline 11 & 51.968 & 40.348 & 63.733 & 9.281 \\
\hline 12 & 50.143 & 44.961 & 64.353 & 9.166 \\
\hline 13 & 46.707 & 48.593 & 64.334 & 9.257 \\
\hline 14 & 43.809 & 52.163 & 66.298 & 8.822 \\
\hline 15 & 40.102 & 55.081 & 67.954 & 9.230 \\
\hline 16 & 36.661 & 57.474 & 70.681 & 10.907 \\
\hline 17 & 33.390 & 57.996 & 74.426 & 14.981 \\
\hline 18 & 31.969 & 58.438 & 79.200 & 19.094 \\
\hline 19 & 29.845 & 57.500 & 83.628 & 17.712 \\
\hline 20 & 29.339 & 57.006 & 88.577 & 19.799 \\
\hline 21 & 26.730 & 57.726 & 92.782 & 16.818 \\
\hline 22 & 22.486 & 57.380 & 95.403 & 10.483 \\
\hline 23 & 18.256 & 58.209 & 97.936 & 10.135 \\
\hline 24 & 14.084 & 60.827 & 98.797 & 9.851 \\
\hline 25 & 9.687 & 62.529 & 100.460 & 9.427 \\
\hline
\end{tabular}




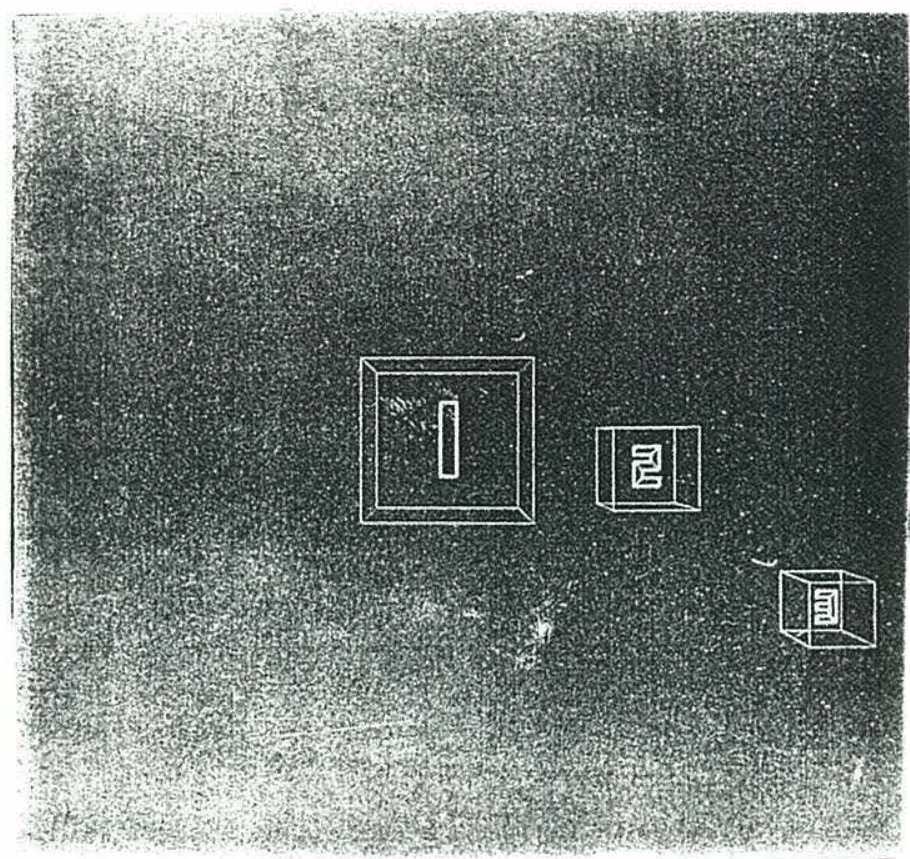

Figure D.7 Course G (operator's display with $60^{\circ}$ FOV)

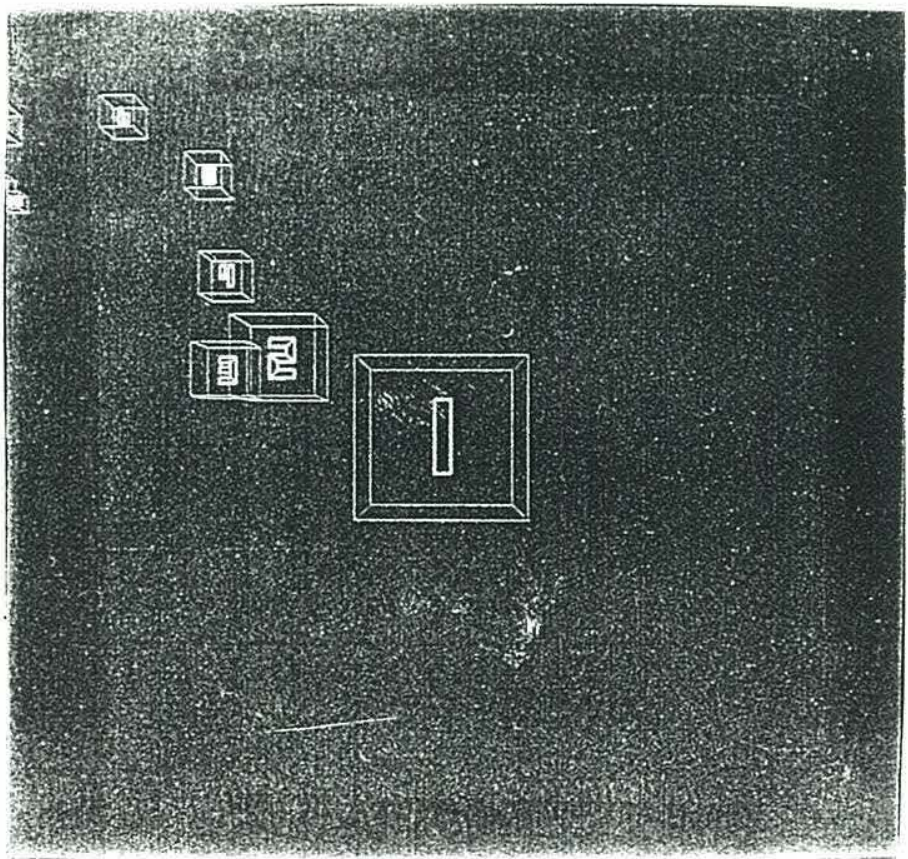

Figure D.8 Course $\mathrm{H}$ (operator's display with $60^{\circ} \mathrm{FOV}$ ) 
Table D.9 Course I

\begin{tabular}{|c|c|c|c|c|}
\hline Target & $\mathrm{x}$ & $y$ & $\mathrm{z}$ & Best $\delta \mathrm{t}$ \\
\hline 1 & 75 & 5 & 50 & N/A \\
\hline 2 & 73.705 & 9.827 & 49.845 & 9.654 \\
\hline 3 & 70.962 & 13.348 & 47.593 & 9.011 \\
\hline 4 & 69.007 & 17.733 & 46.196 & 9.422 \\
\hline 5 & 65.232 & 20.735 & 44.877 & 8.664 \\
\hline 6 & 61.594 & 24.027 & 43.913 & 9.805 \\
\hline 7 & 59.815 & 28.690 & 43.604 & 9.113 \\
\hline 8 & 59.727 & 33.487 & 45.011 & 9.341 \\
\hline 9 & 58.587 & 38.275 & 45.893 & 9.711 \\
\hline 10 & 55.693 & 41.610 & 48.238 & 9.379 \\
\hline 11 & 53.396 & 44.963 & 51.151 & 11.651 \\
\hline 12 & 50.001 & 45.848 & 54.713 & 14.250 \\
\hline 13 & 47.180 & 43.768 & 58.279 & 14.261 \\
\hline 14 & 46.505 & 40.286 & 61.803 & 14.096 \\
\hline 15 & 45.997 & 37.783 & 66.101 & 17.195 \\
\hline 16 & 45.976 & 34.940 & 70.214 & 16.450 \\
\hline 17 & 45.205 & 32.056 & 74.225 & 16.044 \\
\hline 18 & 44.872 & 28.706 & 77.922 & 14.787 \\
\hline 19 & 42.788 & 24.785 & 80.222 & 9.200 \\
\hline 20 & 39.883 & 20.716 & 80.238 & 8.983 \\
\hline 21 & 35.754 & 18.089 & 81.262 & 9.452 \\
\hline 22 & 31.130 & 17.307 & 79.527 & 8.462 \\
\hline 23 & 26.277 & 16.223 & 80.051 & 9.048 \\
\hline 24 & 21.766 & 15.832 & 77.931 & 8.483 \\
\hline 25 & 17.182 & 17.512 & 76.854 & 9.276 \\
\hline
\end{tabular}


Table D.10 Course J

\begin{tabular}{|c|c|c|c|c|}
\hline Target & $\mathrm{x}$ & $\mathrm{y}$ & $\mathrm{z}$ & Best $\delta \mathrm{t}$ \\
\hline 1 & 75 & 5 & 50 & $\mathrm{~N} / \mathrm{A}$ \\
\hline 2 & 74.660 & 9.971 & 50.419 & 9.942 \\
\hline 3 & 74.317 & 14.959 & 50.393 & 10.000 \\
\hline 4 & 74.257 & 19.937 & 50.862 & 9.940 \\
\hline 5 & 73.663 & 24.897 & 51.060 & 9.935 \\
\hline 6 & 72.796 & 29.809 & 50.703 & 9.959 \\
\hline 7 & 71.599 & 34.662 & 50.821 & 9.975 \\
\hline 8 & 70.152 & 39.447 & 50.718 & 9.984 \\
\hline 9 & 69.008 & 44.314 & 50.665 & 9.980 \\
\hline 10 & 67.879 & 49.184 & 50.707 & 10.000 \\
\hline 11 & 66.190 & 53.888 & 50.570 & 9.928 \\
\hline 12 & 63.923 & 58.330 & 50.930 & 9.894 \\
\hline 13 & 61.906 & 62.885 & 51.366 & 9.947 \\
\hline 14 & 59.907 & 67.411 & 52.085 & 9.896 \\
\hline 15 & 58.334 & 72.019 & 53.219 & 9.704 \\
\hline 16 & 57.239 & 76.807 & 54.158 & 9.770 \\
\hline 17 & 56.324 & 81.488 & 55.659 & 9.536 \\
\hline 18 & 55.924 & 86.374 & 56.640 & 9.742 \\
\hline 19 & 55.050 & 91.283 & 57.016 & 9.935 \\
\hline 20 & 54.653 & 96.261 & 57.262 & 9.940 \\
\hline 21 & 54.405 & 101.250 & 57.047 & 9.984 \\
\hline 22 & 54.546 & 106.237 & 56.713 & 9.947 \\
\hline 23 & 54.785 & 111.159 & 55.866 & 9.852 \\
\hline 24 & 55.274 & 115.948 & 54.514 & 9.612 \\
\hline 25 & 55.208 & 120.760 & 53.159 & 9.566 \\
\hline
\end{tabular}




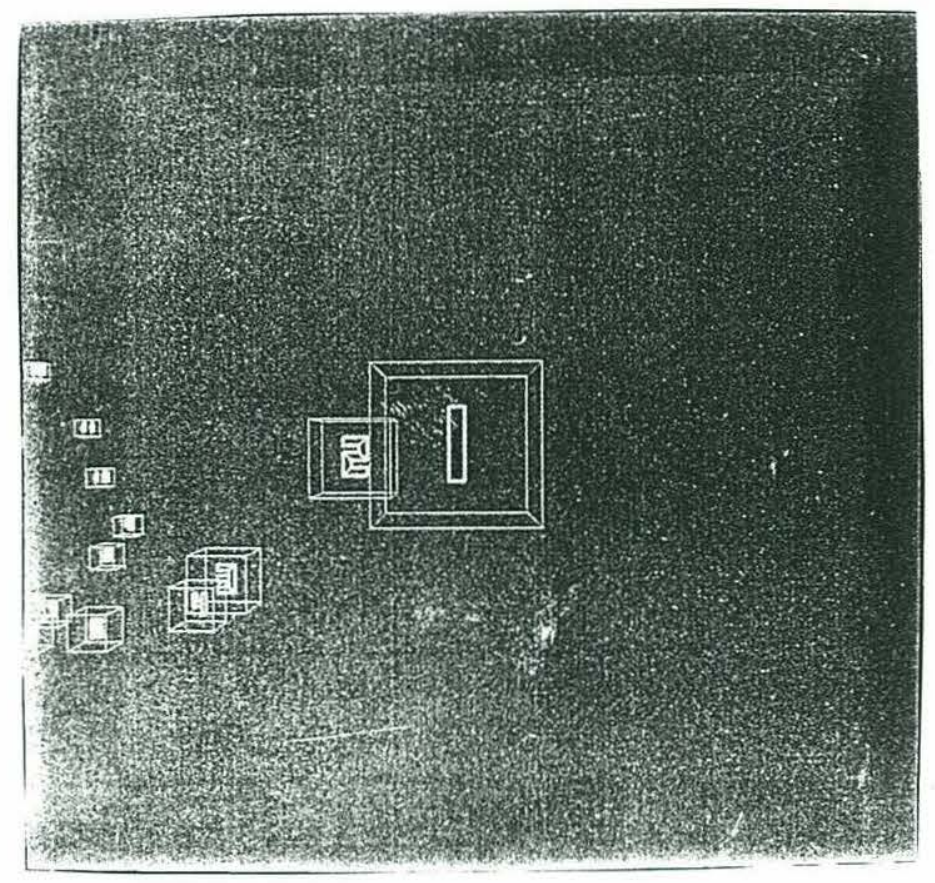

Figure D.9 Course I (operator's display with $60^{\circ} \mathrm{FOV}$ )

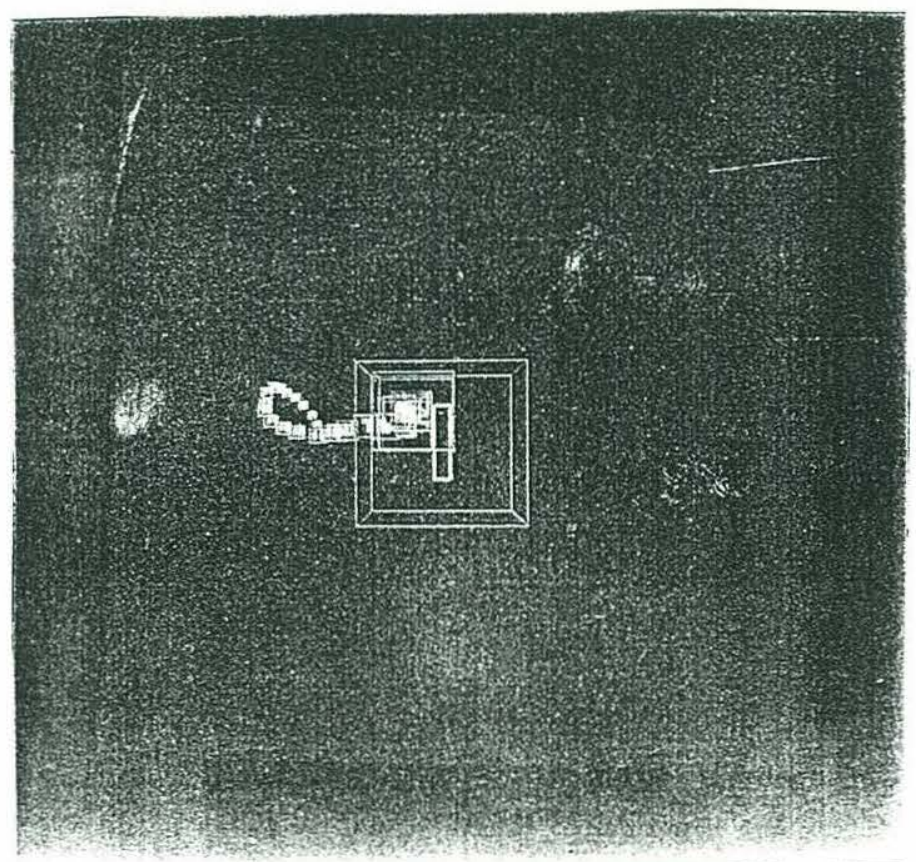

Figure D.10 Course J (operator's display with $60^{\circ} \mathrm{FOV}$ ) 
Table D.11 Course K

\begin{tabular}{|c|c|c|c|c|}
\hline Target & $\mathrm{x}$ & $\mathrm{y}$ & $\mathrm{z}$ & Best $\delta \mathrm{t}$ \\
\hline 1 & 75 & 5 & 50 & N/A \\
\hline 2 & 74.486 & 9.942 & 50.561 & 9.884 \\
\hline 3 & 73.720 & 14.801 & 51.459 & 9.825 \\
\hline 4 & 73.536 & 19.731 & 52.270 & 9.800 \\
\hline 5 & 73.212 & 24.651 & 53.100 & 9.857 \\
\hline 6 & 72.381 & 29.517 & 53.893 & 9.822 \\
\hline 7 & 71.286 & 34.369 & 54.406 & 9.933 \\
\hline 8 & 69.809 & 39.111 & 54.981 & 9.903 \\
\hline 9 & 67.753 & 43.558 & 55.978 & 9.723 \\
\hline 10 & 66.055 & 48.008 & 57.501 & 9.500 \\
\hline 11 & 64.013 & 52.139 & 59.440 & 9.186 \\
\hline 12 & 61.632 & 56.088 & 61.373 & 9.196 \\
\hline 13 & 59.818 & 60.266 & 63.436 & 9.039 \\
\hline 14 & 58.333 & 64.362 & 65.889 & 9.812 \\
\hline 15 & 57.201 & 68.246 & 68.827 & 11.755 \\
\hline 16 & 56.308 & 72.084 & 71.905 & 12.311 \\
\hline 17 & 55.328 & 75.833 & 75.064 & 12.636 \\
\hline 18 & 53.753 & 79.760 & 77.729 & 10.659 \\
\hline 19 & 52.415 & 83.600 & 80.639 & 11.639 \\
\hline 20 & 51.576 & 87.377 & 83.806 & 12.669 \\
\hline 21 & 50.316 & 90.714 & 87.309 & 14.012 \\
\hline 22 & 48.508 & 93.759 & 90.839 & 14.118 \\
\hline 23 & 47.425 & 96.682 & 94.748 & 15.637 \\
\hline 24 & 46.026 & 99.466 & 98.659 & 15.643 \\
\hline 25 & 44.374 & 101.705 & 102.813 & 16.618 \\
\hline
\end{tabular}


Table D.12 Course L

\begin{tabular}{|c|c|c|c|c|}
\hline Target & $\mathrm{x}$ & $\mathrm{y}$ & $\mathrm{z}$ & Best $\delta \mathrm{t}$ \\
\hline 1 & 75 & 5 & 50 & N/A \\
\hline 2 & 74.980 & 9.983 & 50.415 & 9.965 \\
\hline 3 & 75.020 & 14.977 & 50.180 & 9.988 \\
\hline 4 & 75.075 & 19.976 & 50.095 & 9.999 \\
\hline 5 & 74.731 & 24.919 & 49.429 & 9.879 \\
\hline 6 & 74.408 & 29.763 & 48.232 & 9.709 \\
\hline 7 & 73.557 & 34.610 & 47.343 & 9.786 \\
\hline 8 & 72.325 & 39.396 & 46.586 & 9.855 \\
\hline 9 & 71.518 & 44.192 & 45.423 & 9.690 \\
\hline 10 & 70.658 & 48.955 & 44.170 & 9.680 \\
\hline 11 & 69.929 & 53.561 & 42.368 & 9.325 \\
\hline 12 & 69.330 & 58.230 & 40.681 & 9.410 \\
\hline 13 & 68.508 & 63.000 & 39.427 & 9.672 \\
\hline 14 & 67.425 & 67.842 & 38.810 & 9.911 \\
\hline 15 & 66.003 & 72.636 & 38.769 & 9.974 \\
\hline 16 & 64.808 & 77.489 & 38.898 & 9.986 \\
\hline 17 & 63.704 & 82.364 & 39.015 & 9.995 \\
\hline 18 & 62.880 & 87.270 & 38.511 & 9.932 \\
\hline 19 & 61.743 & 92.015 & 37.420 & 9.738 \\
\hline 20 & 60.883 & 96.742 & 36.035 & 9.594 \\
\hline 21 & 60.588 & 101.410 & 34.268 & 9.297 \\
\hline 22 & 60.240 & 106.245 & 33.043 & 9.695 \\
\hline 23 & 60.293 & 111.017 & 31.552 & 9.516 \\
\hline 24 & 60.272 & 115.746 & 29.930 & 9.458 \\
\hline 25 & 60.149 & 120.359 & 28.004 & 9.226 \\
\hline
\end{tabular}




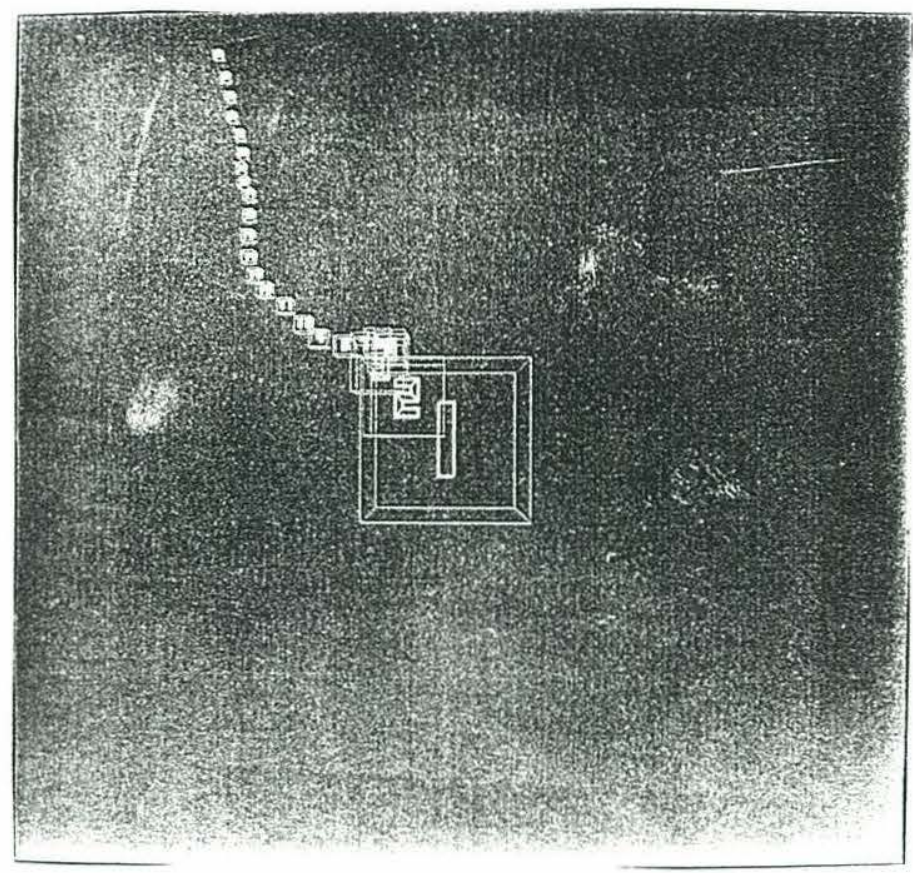

Figure D.11 Course K (operator's display with $60^{\circ} \mathrm{FOV}$ )

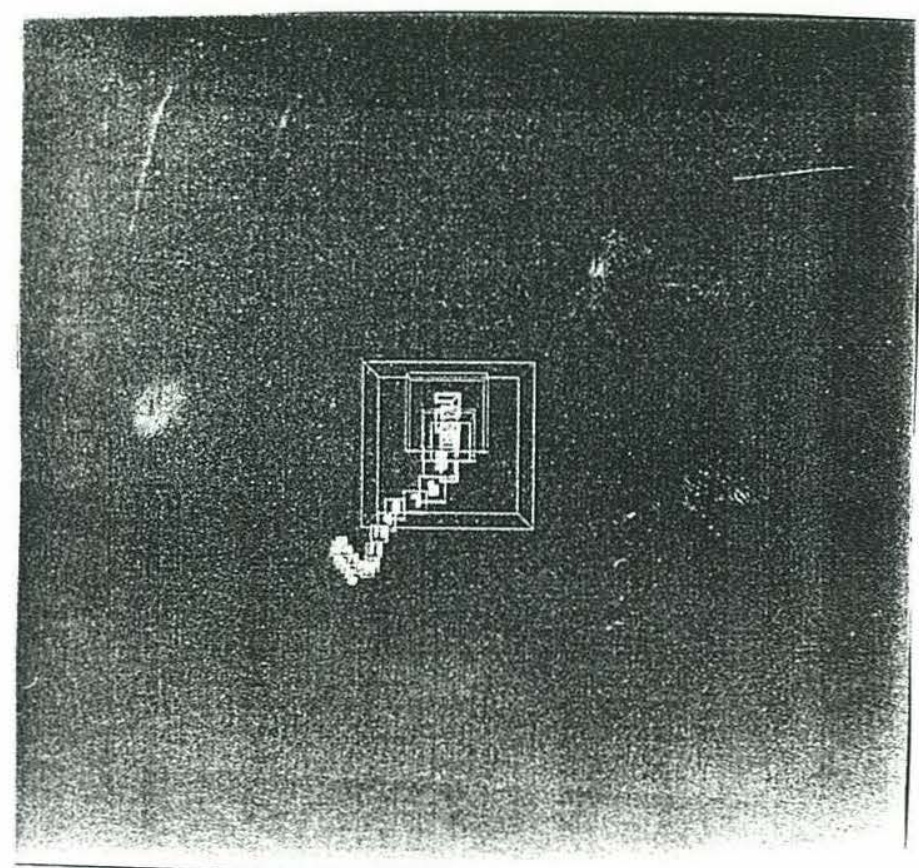

Figure D.12 Course L (operator's display with $60^{\circ} \mathrm{FOV}$ ) 
Table D.13 Course M

\begin{tabular}{|c|c|c|c|c|}
\hline Target & $\mathrm{x}$ & $\mathrm{y}$ & $\mathrm{z}$ & Best $\delta \mathrm{t}$ \\
\hline 1 & 75 & 5 & 50 & N/A \\
\hline 2 & 75.628 & 9.917 & 50.658 & 9.835 \\
\hline 3 & 75.386 & 14.903 & 50.928 & 9.835 \\
\hline 4 & 75.174 & 19.671 & 52.420 & 9.544 \\
\hline 5 & 74.717 & 24.294 & 54.270 & 9.278 \\
\hline 6 & 73.107 & 28.957 & 55.080 & 9.601 \\
\hline 7 & 70.368 & 33.111 & 55.573 & 9.637 \\
\hline 8 & 67.719 & 37.123 & 56.946 & 9.615 \\
\hline 9 & 64.808 & 41.067 & 57.934 & 9.787 \\
\hline 10 & 62.750 & 45.189 & 59.876 & 9.073 \\
\hline 11 & 60.955 & 48.851 & 62.768 & 11.570 \\
\hline 12 & 58.810 & 52.496 & 65.436 & 10.670 \\
\hline 13 & 56.894 & 56.769 & 67.187 & 9.327 \\
\hline 14 & 55.265 & 61.062 & 69.167 & 9.169 \\
\hline 15 & 54.509 & 65.646 & 71.015 & 9.134 \\
\hline 16 & 54.231 & 70.151 & 73.167 & 8.993 \\
\hline 17 & 54.949 & 74.059 & 76.202 & 12.139 \\
\hline 18 & 55.805 & 78.131 & 78.975 & 11.092 \\
\hline 19 & 55.413 & 82.593 & 81.196 & 8.885 \\
\hline 20 & 54.037 & 86.945 & 83.236 & 8.962 \\
\hline 21 & 53.306 & 91.569 & 84.993 & 9.265 \\
\hline 22 & 51.427 & 96.153 & 85.668 & 9.709 \\
\hline 23 & 49.775 & 100.734 & 86.802 & 9.734 \\
\hline 24 & 48.772 & 105.627 & 86.583 & 9.845 \\
\hline 25 & 48.036 & 110.571 & 86.428 & 9.981 \\
\hline
\end{tabular}


Table D.14 Course N

\begin{tabular}{|c|c|c|c|c|}
\hline Target & $\mathrm{x}$ & $\mathrm{y}$ & $\mathrm{z}$ & Best $\delta t$ \\
\hline 1 & 75 & 5 & 50 & N/A \\
\hline 2 & 75.836 & 9.874 & 50.737 & 9.752 \\
\hline 3 & 77.010 & 14.566 & 52.005 & 9.649 \\
\hline 4 & 77.896 & 19.139 & 53.823 & 9.301 \\
\hline 5 & 79.593 & 23.580 & 55.370 & 9.378 \\
\hline 6 & 81.786 & 28.060 & 55.718 & 9.917 \\
\hline 7 & 83.242 & 32.567 & 57.322 & 9.357 \\
\hline 8 & 85.209 & 36.764 & 59.197 & 9.209 \\
\hline 9 & 86.375 & 41.499 & 60.302 & 9.599 \\
\hline 10 & 88.743 & 45.902 & 60.362 & 9.663 \\
\hline 11 & 90.494 & 50.584 & 60.486 & 9.905 \\
\hline 12 & 91.875 & 55.374 & 60.874 & 9.939 \\
\hline 13 & 92.059 & 60.311 & 61.644 & 9.591 \\
\hline 14 & 91.768 & 65.298 & 61.853 & 9.945 \\
\hline 15 & 90.631 & 70.150 & 62.256 & 9.822 \\
\hline 16 & 89.297 & 74.966 & 62.424 & 9.986 \\
\hline 17 & 86.789 & 79.278 & 62.770 & 9.653 \\
\hline 18 & 84.275 & 83.598 & 62.902 & 9.997 \\
\hline 19 & 81.106 & 87.449 & 63.255 & 9.849 \\
\hline 20 & 78.904 & 91.933 & 63.470 & 9.728 \\
\hline 21 & 76.920 & 96.326 & 64.797 & 9.633 \\
\hline 22 & 74.316 & 100.527 & 65.557 & 9.796 \\
\hline 23 & 72.143 & 105.028 & 65.683 & 9.949 \\
\hline 24 & 70.432 & 109.626 & 64.715 & 9.780 \\
\hline 25 & 68.153 & 113.963 & 63.718 & 9.723 \\
\hline
\end{tabular}




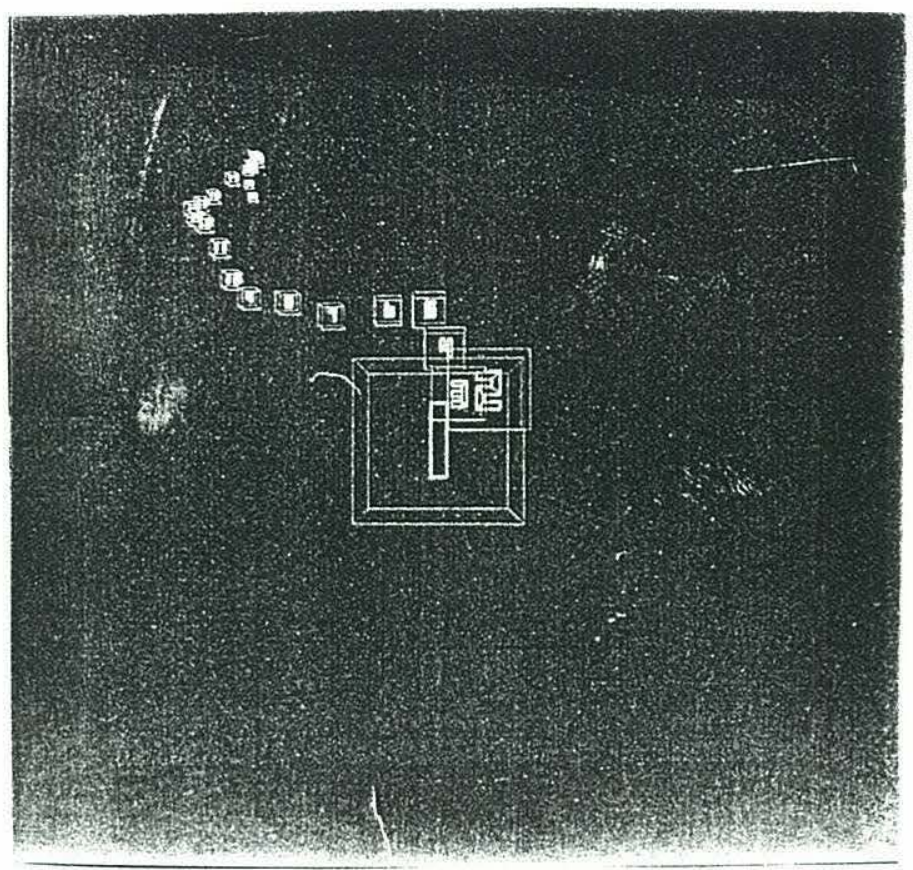

Figure D.13 Course M (operator's display with $60^{\circ} \mathrm{FOV}$ )

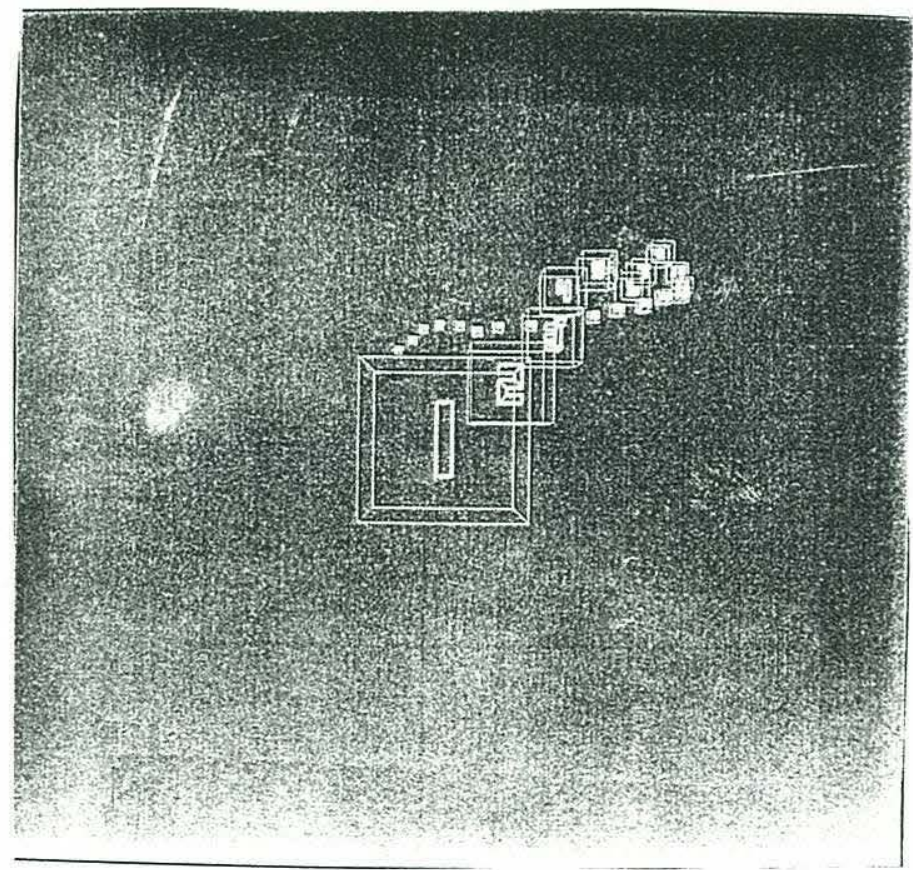

Figure D.14 Course N (operator's display with $60^{\circ} \mathrm{FOV}$ ) 
Table D.15 Course O

\begin{tabular}{|c|c|c|c|c|}
\hline Target & $\mathrm{x}$ & $\mathrm{y}$ & $z$ & Best $\delta t$ \\
\hline 1 & 75 & 5 & 50 & N/A \\
\hline 2 & 75.910 & 9.822 & 50.957 & 9.651 \\
\hline 3 & 77.211 & 14.460 & 52.299 & 9.602 \\
\hline 4 & 78.500 & 18.826 & 54.367 & 9.105 \\
\hline 5 & 78.682 & 23.509 & 56.109 & 9.130 \\
\hline 6 & 78.331 & 28.250 & 57.657 & 9.453 \\
\hline 7 & 79.284 & 32.909 & 59.205 & 9.185 \\
\hline 8 & 80.718 & 37.669 & 59.737 & 9.897 \\
\hline 9 & 82.527 & 42.322 & 60.019 & 9.952 \\
\hline 10 & 84.463 & 46.929 & 60.170 & 9.991 \\
\hline 11 & 86.928 & 51.277 & 60.311 & 9.927 \\
\hline 12 & 89.000 & 55.811 & 59.923 & 9.936 \\
\hline 13 & 91.435 & 60.063 & 58.930 & 9.756 \\
\hline 14 & 93.206 & 64.739 & 58.944 & 9.864 \\
\hline 15 & 96.054 & 68.755 & 58.072 & 9.523 \\
\hline 16 & 98.649 & 73.023 & 57.847 & 9.966 \\
\hline 17 & 100.986 & 77.376 & 57.080 & 9.867 \\
\hline 18 & 104.258 & 80.745 & 55.364 & 9.074 \\
\hline 19 & 108.280 & 83.431 & 54.096 & 9.457 \\
\hline 20 & 111.762 & 87.010 & 53.838 & 9.831 \\
\hline 21 & 115.113 & 90.688 & 54.334 & 9.950 \\
\hline 22 & 118.488 & 94.286 & 53.521 & 9.864 \\
\hline 23 & 120.980 & 98.614 & 53.754 & 9.792 \\
\hline 24 & 122.835 & 103.247 & 54.071 & 9.886 \\
\hline 25 & 124.504 & 107.871 & 53.158 & 9.806 \\
\hline
\end{tabular}


Table D.16 Course P

\begin{tabular}{|c|c|c|c|c|}
\hline Target & $\mathrm{x}$ & $\mathrm{y}$ & $\mathrm{z}$ & Best $\delta t$ \\
\hline 1 & 75 & 5 & 50 & N/A \\
\hline 2 & 72.598 & 9.372 & 49.658 & 9.584 \\
\hline 3 & 69.070 & 12.001 & 47.283 & 9.501 \\
\hline 4 & 64.278 & 13.316 & 46.731 & 8.949 \\
\hline 5 & 59.900 & 13.069 & 44.328 & 9.609 \\
\hline 6 & 54.955 & 13.750 & 44.040 & 9.983 \\
\hline 7 & 50.197 & 12.432 & 43.248 & 9.344 \\
\hline 8 & 45.239 & 12.926 & 42.829 & 9.532 \\
\hline 9 & 40.819 & 12.034 & 44.988 & 8.637 \\
\hline 10 & 38.922 & 10.074 & 49.179 & 16.763 \\
\hline 11 & 39.445 & 7.725 & 53.562 & 17.531 \\
\hline 12 & 39.178 & 7.113 & 58.517 & 19.820 \\
\hline 13 & 37.208 & 8.030 & 63.020 & 18.011 \\
\hline 14 & 34.728 & 10.445 & 66.628 & 14.434 \\
\hline 15 & 33.378 & 14.175 & 69.671 & 12.174 \\
\hline 16 & 31.837 & 16.656 & 73.730 & 16.233 \\
\hline 17 & 32.710 & 18.362 & 78.348 & 18.473 \\
\hline 18 & 31.547 & 19.258 & 83.127 & 19.118 \\
\hline 19 & 29.540 & 18.207 & 87.585 & 17.829 \\
\hline 20 & 26.954 & 15.938 & 91.212 & 14.510 \\
\hline 21 & 23.758 & 14.134 & 94.609 & 13.584 \\
\hline 22 & 19.241 & 13.240 & 96.559 & 8.860 \\
\hline 23 & 14.933 & 14.923 & 98.456 & 9.114 \\
\hline 24 & 10.241 & 16.585 & 98.932 & 9.935 \\
\hline 25 & 5.979 & 18.058 & 101.093 & 8.962 \\
\hline
\end{tabular}




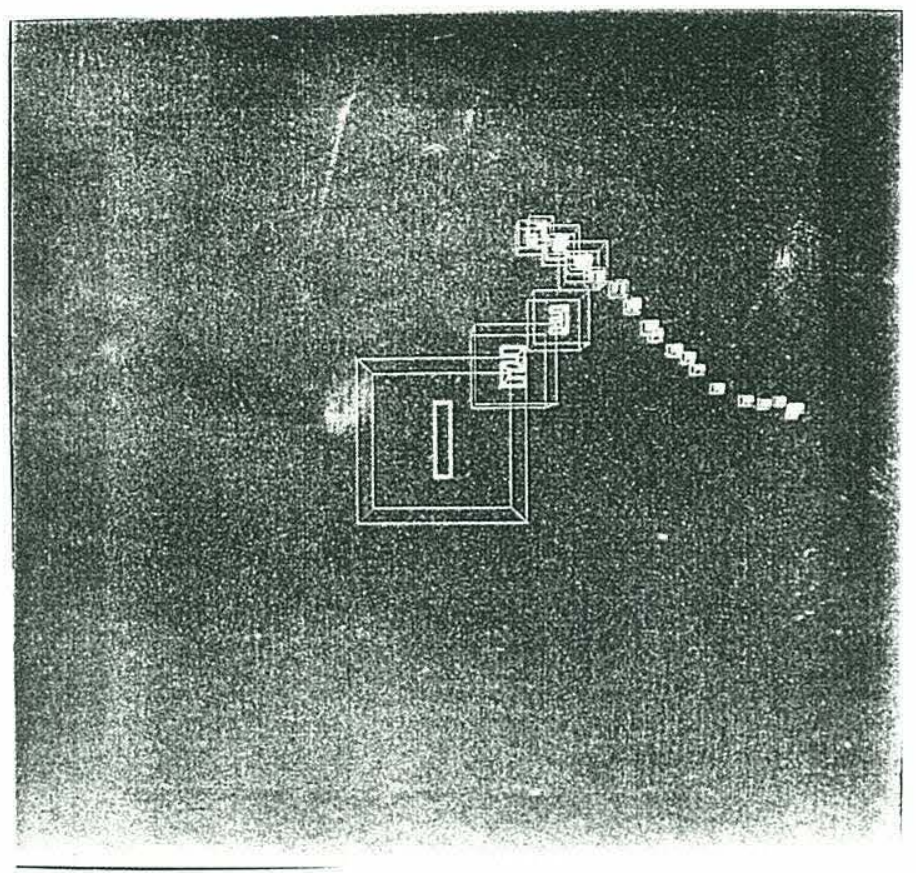

Figure D.15 Course O (operator's display with $60^{\circ}$ FOV)

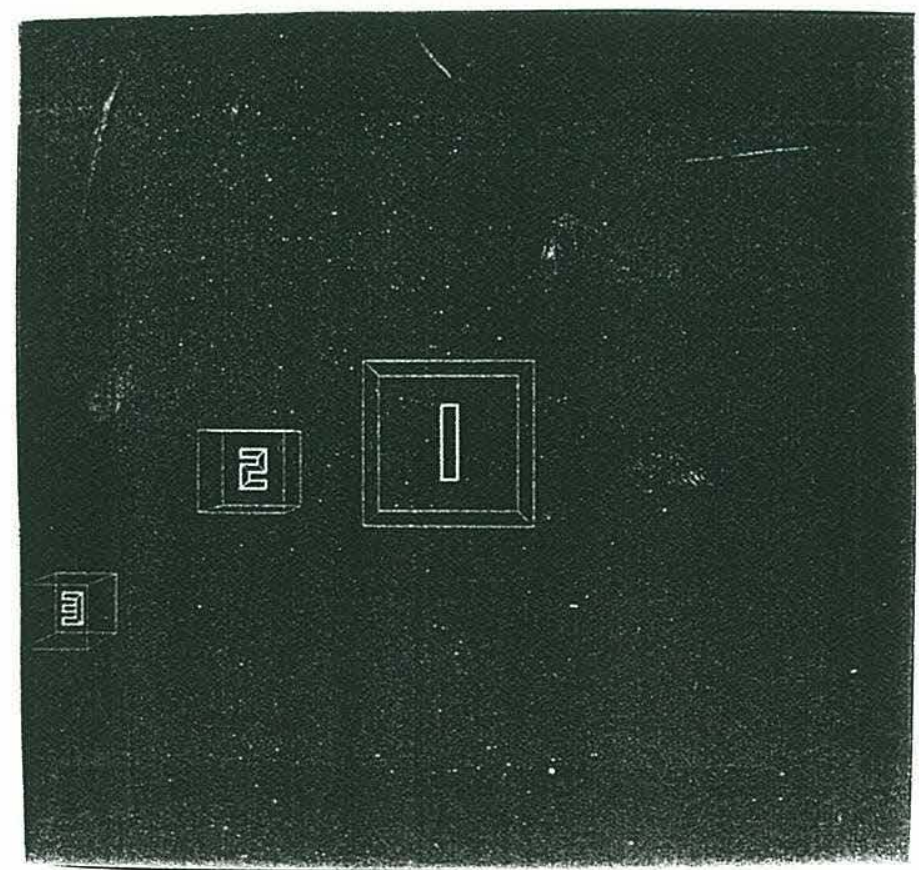

Figure D.16 Course P (operator's display with $60^{\circ}$ FOV) 
Table D.17 Course Q

\begin{tabular}{|c|c|c|c|c|}
\hline Target & $\mathrm{x}$ & $\mathrm{y}$ & $\mathrm{z}$ & Best $\delta t$ \\
\hline 1 & 75 & 5 & 50 & N/A \\
\hline 2 & 76.965 & 9.488 & 50.999 & 9.010 \\
\hline 3 & 78.914 & 14.085 & 51.257 & 9.987 \\
\hline 4 & 80.057 & 18.154 & 53.928 & 10.684 \\
\hline 5 & 81.442 & 21.353 & 57.512 & 14.338 \\
\hline 6 & 84.785 & 23.831 & 60.286 & 11.093 \\
\hline 7 & 89.534 & 25.325 & 60.742 & 9.566 \\
\hline 8 & 94.093 & 26.833 & 62.137 & 9.558 \\
\hline 9 & 97.370 & 30.596 & 62.454 & 9.227 \\
\hline 10 & 97.983 & 35.556 & 62.305 & 9.426 \\
\hline 11 & 98.032 & 40.348 & 63.733 & 9.281 \\
\hline 12 & 99.857 & 44.961 & 64.353 & 9.166 \\
\hline 13 & 103.293 & 48.593 & 64.334 & 9.257 \\
\hline 14 & 106.191 & 52.163 & 66.298 & 8.822 \\
\hline 15 & 109.898 & 55.081 & 67.954 & 9.230 \\
\hline 16 & 113.339 & 57.474 & 70.681 & 10.907 \\
\hline 17 & 116.610 & 57.996 & 74.426 & 14.981 \\
\hline 18 & 118.031 & 58.438 & 79.200 & 19.094 \\
\hline 19 & 120.155 & 57.500 & 83.628 & 17.712 \\
\hline 20 & 120.661 & 57.006 & 88.577 & 19.799 \\
\hline 21 & 123.270 & 57.726 & 92.782 & 16.818 \\
\hline 22 & 127.514 & 57.380 & 95.403 & 10.483 \\
\hline 23 & 131.744 & 58.209 & 97.936 & 10.135 \\
\hline 24 & 135.916 & 60.827 & 98.797 & 9.851 \\
\hline 25 & 140.313 & 62.529 & 100.460 & 9.427 \\
\hline
\end{tabular}


Table D.18 Course R

\begin{tabular}{|c|c|c|c|c|}
\hline Target & $\mathrm{x}$ & $\mathrm{y}$ & z & Best $\delta t$ \\
\hline 1 & 75 & 5 & 50 & N/A \\
\hline 2 & 76.295 & 9.827 & 49.845 & 9.654 \\
\hline 3 & 79.038 & 13.348 & 47.593 & 9.011 \\
\hline 4 & 80.993 & 17.733 & 46.196 & 9.422 \\
\hline 5 & 84.768 & 20.735 & 44.877 & 8.664 \\
\hline 6 & 88.406 & 24.027 & 43.913 & 9.805 \\
\hline 7 & 90.185 & 28.690 & 43.604 & 9.113 \\
\hline 8 & 90.273 & 33.487 & 45.011 & 9.341 \\
\hline 9 & 91.413 & 38.275 & 45.893 & 9.711 \\
\hline 10 & 94.307 & 41.610 & 48.238 & 9.379 \\
\hline 11 & 96.604 & 44.963 & 51.151 & 11.651 \\
\hline 12 & 99.999 & 45.848 & 54.713 & 14.250 \\
\hline 13 & 102.820 & 43.768 & 58.279 & 14.261 \\
\hline 14 & 103.495 & 40.286 & 61.803 & 14.096 \\
\hline 15 & 104.003 & 37.783 & 66.101 & 17.195 \\
\hline 16 & 104.024 & 34.940 & 70.214 & 16.450 \\
\hline 17 & 104.795 & 32.056 & 74.225 & 16.044 \\
\hline 18 & 105.128 & 28.706 & 77.922 & 14.787 \\
\hline 19 & 107.212 & 24.785 & 80.222 & 9.200 \\
\hline 20 & 110.117 & 20.716 & 80.238 & 8.983 \\
\hline 21 & 114.246 & 18.089 & 81.262 & 9.452 \\
\hline 22 & 118.870 & 17.307 & 79.527 & 8.462 \\
\hline 23 & 123.723 & 16.223 & 80.051 & 9.048 \\
\hline 24 & 128.234 & 15.832 & 77.931 & 8.483 \\
\hline 25 & 132.818 & 17.512 & 76.854 & 9.276 \\
\hline
\end{tabular}




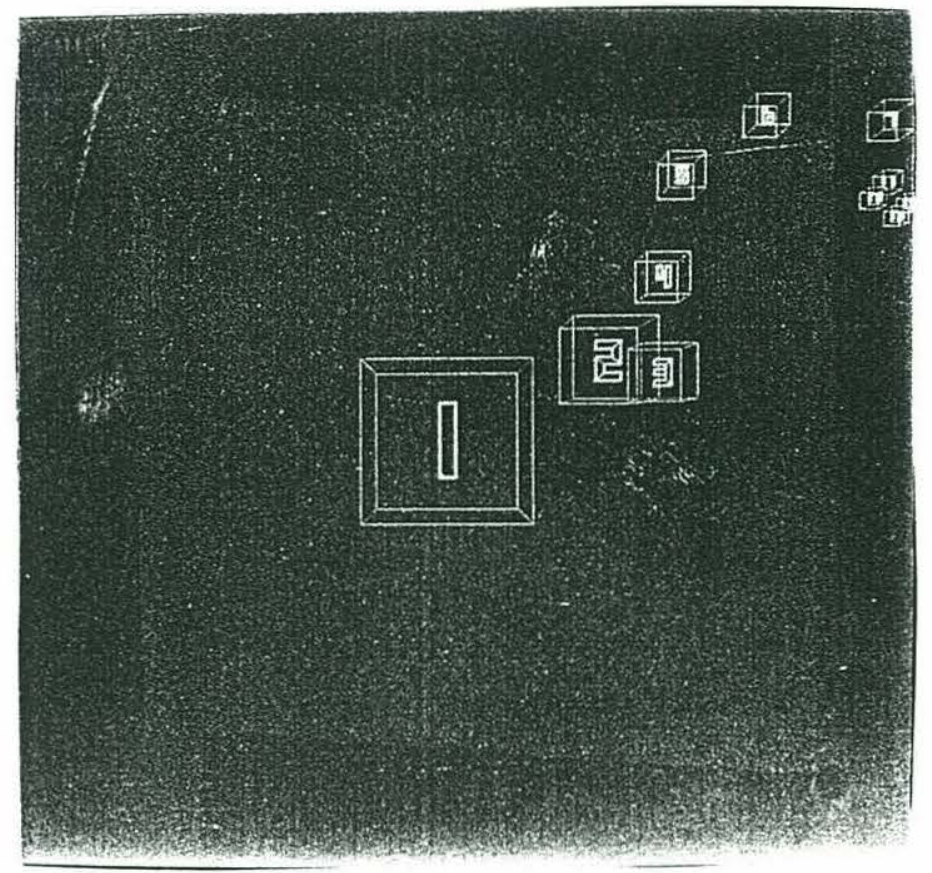

Figure D.17 Course Q (operator's display with $60^{\circ} \mathrm{FOV}$ )

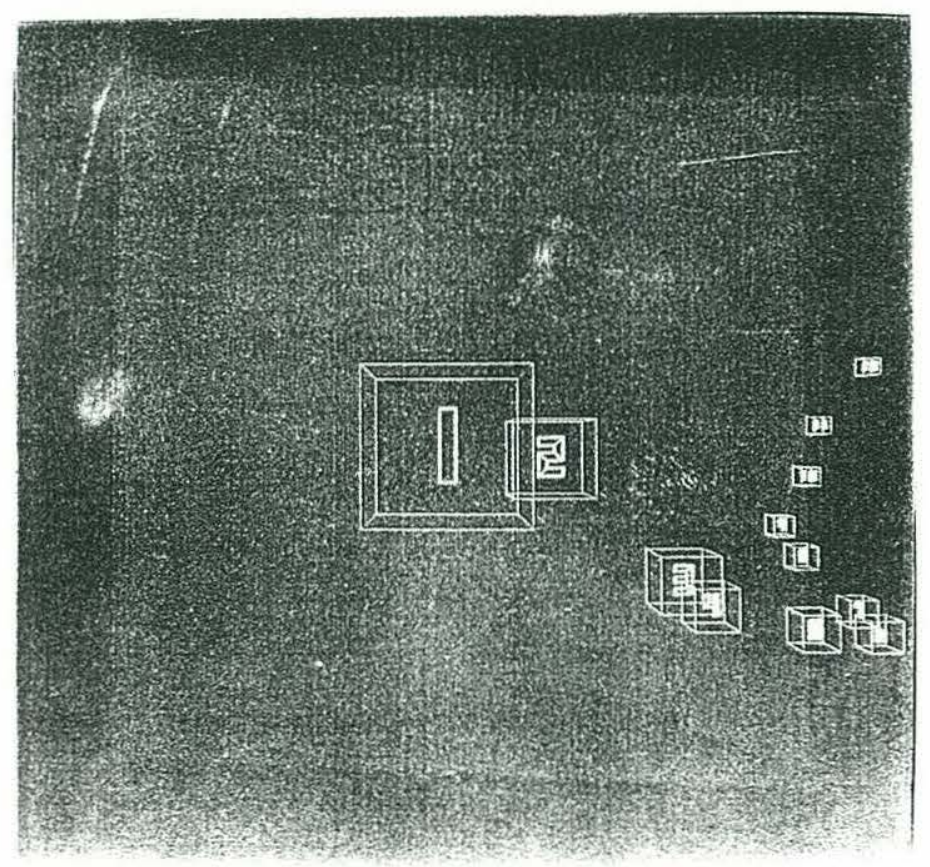

Figure D.18 Course R (operator's display with $60^{\circ} \mathrm{FOV}$ ) 
Table D.19 Course X

\begin{tabular}{|c|c|c|c|}
\hline Target & $\mathrm{x}$ & $\mathrm{y}$ & $\mathrm{z}$ \\
\hline 1 & 75 & 5 & 50 \\
\hline 2 & 75.415 & 9.975 & 50.274 \\
\hline 3 & 75.804 & 14.960 & 50.278 \\
\hline 4 & 76.682 & 19.878 & 50.081 \\
\hline 5 & 77.443 & 24.817 & 49.909 \\
\hline 6 & 78.169 & 29.761 & 50.092 \\
\hline 7 & 79.052 & 34.674 & 49.800 \\
\hline 8 & 80.541 & 39.441 & 50.037 \\
\hline 9 & 81.479 & 44.352 & 50.041 \\
\hline 10 & 82.073 & 49.286 & 50.595 \\
\hline 11 & 82.721 & 54.141 & 51.598 \\
\hline 12 & 83.762 & 58.762 & 53.199 \\
\hline 13 & 85.367 & 62.995 & 55.321 \\
\hline 14 & 87.501 & 66.919 & 57.568 \\
\hline 15 & 89.307 & 71.089 & 59.653 \\
\hline 16 & 91.327 & 75.215 & 61.627 \\
\hline 17 & 93.395 & 79.426 & 63.357 \\
\hline 18 & 95.436 & 83.409 & 65.587 \\
\hline 19 & 98.061 & 87.359 & 67.170 \\
\hline 20 & 100.328 & 91.501 & 68.815 \\
\hline 21 & 102.772 & 95.558 & 70.417 \\
\hline 22 & 105.399 & 99.343 & 72.359 \\
\hline 23 & 107.766 & 103.514 & 73.773 \\
\hline 24 & 109.633 & 107.777 & 75.602 \\
\hline 25 & 111.801 & 111.855 & 77.516 \\
\hline
\end{tabular}




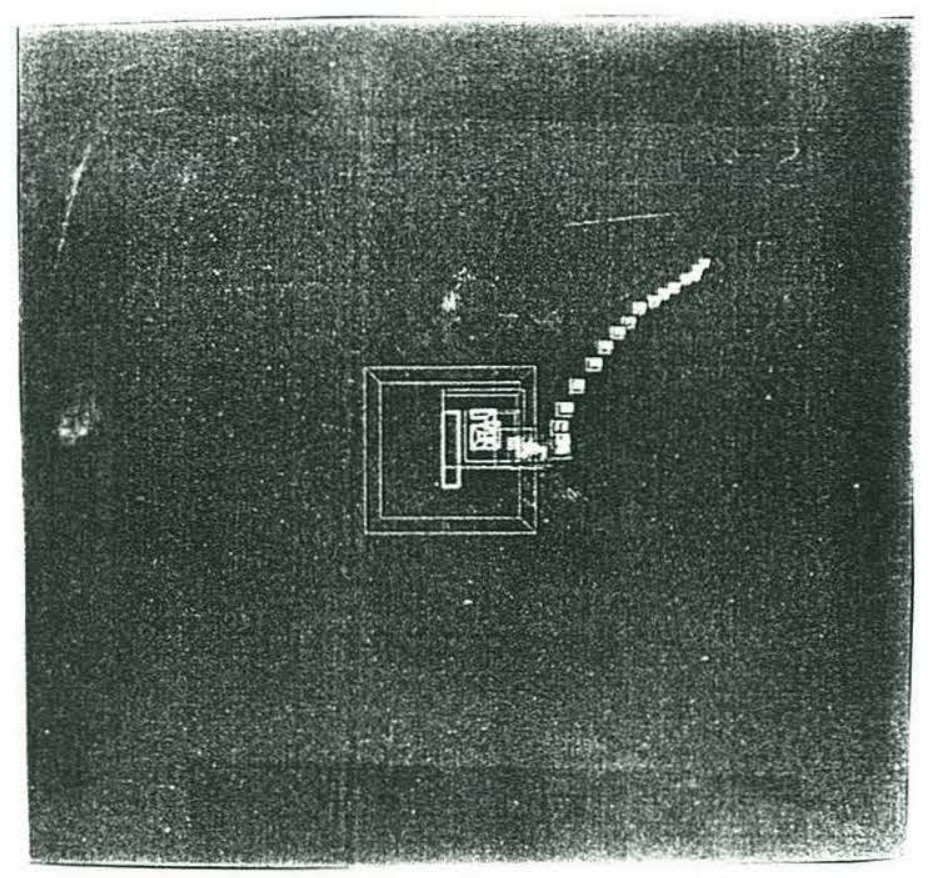

Figure D.19 Course X (operator's display with $60^{\circ} \mathrm{FOV}$ )

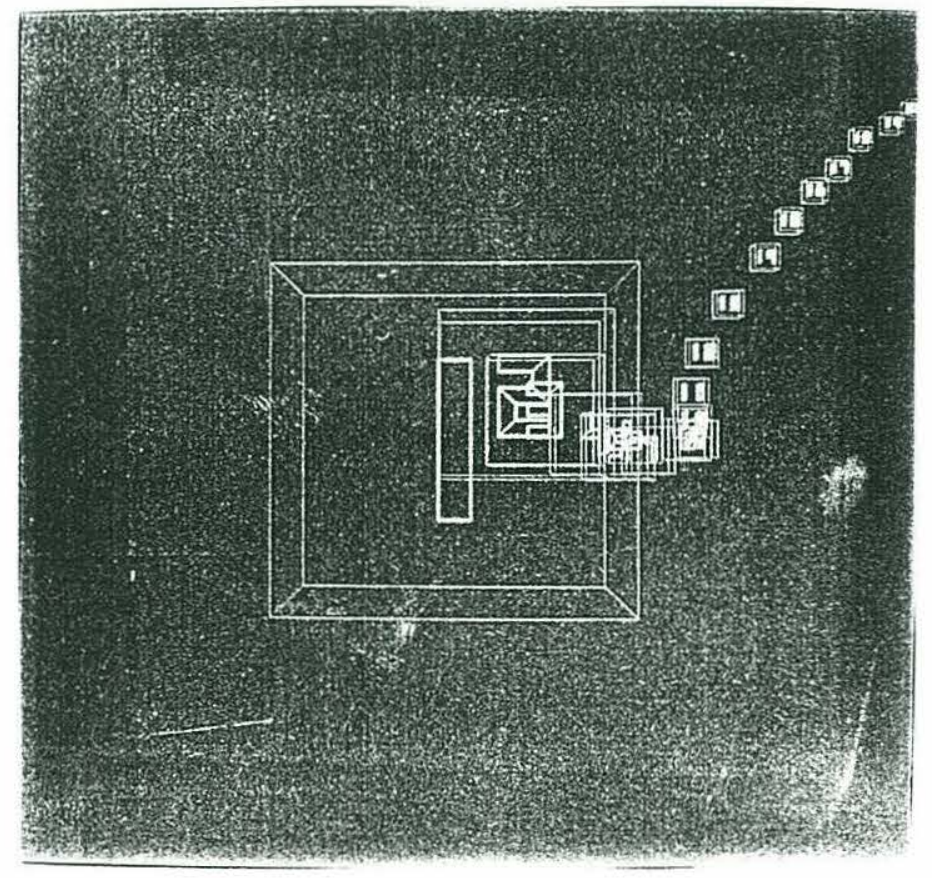

Figure D.20 Course X (operator's display with $30^{\circ}$ FOV) 


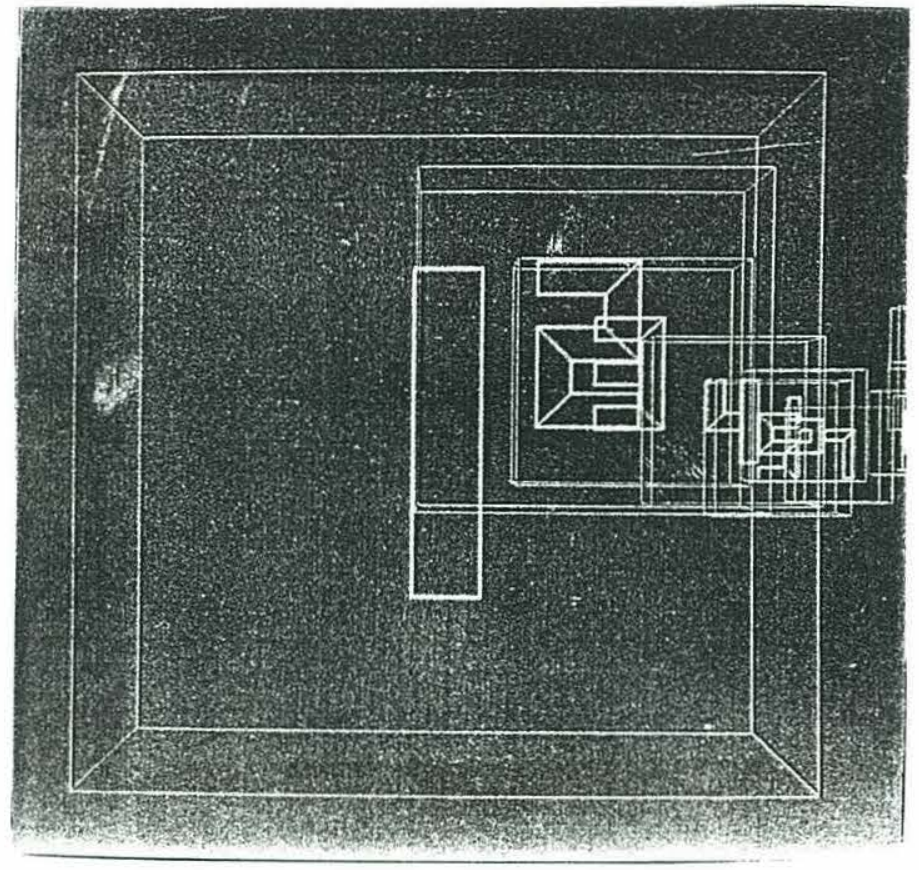

Figure D.21 Course X (operator's display with $15^{\circ} \mathrm{FOV}$ ) 


\section{APPENDIX E}

\section{Raw Data}

Tables E.1 through E.12 list the data for each of the four operators over all seven tests. The tabulated values are the time differences between successive targets in seconds. Value 1 is the time difference between hitting targets 1 and 2, value 2 for targets 2 and 3 , etc. The total for each column is indicated at the bottom of the column. For all tests the

course letter is specified at the top of its respective column. For Test 1 the operator's field of view is also shown. 
Table E.1 Operator I, Test 1

\begin{tabular}{|c|c|c|c|c|c|c|c|c|c|}
\hline & \multicolumn{3}{|c|}{$60^{\circ} \mathrm{FOV}$} & \multicolumn{3}{|c|}{$30^{\circ} \mathrm{FOV}$} & \multicolumn{3}{|c|}{$15^{\circ} \mathrm{FOV}$} \\
\hline & $\mathrm{A}$ & D & $\mathrm{G}$ & B & $E$ & $\mathrm{H}$ & $\mathrm{C}$ & $\mathrm{F}$ & I \\
\hline 1 & 16 & 16 & 13 & 55 & 53 & 35 & 12 & 53 & 42 \\
\hline 2 & 10 & 17 & 39 & 50 & 59 & 38 & 39 & 90 & 49 \\
\hline 3 & 14 & 15 & 16 & 33 & 67 & 101 & 28 & 74 & 29 \\
\hline 4 & 15 & 37 & 28 & 40 & 78 & 59 & 33 & 31 & 45 \\
\hline 5 & 15 & 22 & 32 & 36 & 79 & 48 & 35 & 82 & 79 \\
\hline 6 & 15 & 22 & 22 & 33 & 55 & 34 & 75 & 50 & 43 \\
\hline 7 & 14 & 15 & 14 & 33 & 27 & 23 & 72 & 96 & 32 \\
\hline 8 & 11 & 17 & 28 & 194 & 25 & 39 & 83 & 60 & 33 \\
\hline 9 & 11 & 27 & 129 & 48 & 11 & 11 & 76 & 21 & 219 \\
\hline 10 & 11 & 35 & 88 & 64 & 32 & 36 & 92 & 33 & 27 \\
\hline 11 & 18 & 29 & 180 & 31 & 82 & 22 & 89 & 43 & 189 \\
\hline 12 & 17 & 24 & 67 & 116 & 37 & 41 & 51 & 31 & 221 \\
\hline 13 & 15 & 18 & 34 & 75 & 14 & 106 & 63 & 19 & 46 \\
\hline 14 & 14 & 20 & 18 & 39 & 68 & 44 & 69 & 61 & 47 \\
\hline 15 & 46 & 19 & 25 & 73 & 69 & 94 & 32 & 26 & 59 \\
\hline 16 & 36 & 49 & 44 & 77 & 157 & 52 & 43 & 93 & 109 \\
\hline 17 & 16 & 21 & 78 & 50 & 38 & 195 & 56 & 33 & 114 \\
\hline 18 & 15 & 31 & 93 & 24 & 14 & 48 & 42 & 22 & 25 \\
\hline 19 & 13 & 18 & 104 & 51 & 21 & 46 & 89 & 25 & 41 \\
\hline 20 & 12 & 16 & 38 & 85 & 47 & 60 & 35 & 21 & 83 \\
\hline 21 & 11 & 21 & 51 & 30 & 17 & 57 & 40 & 24 & 22 \\
\hline 22 & 11 & 16 & 38 & 47 & 11 & 26 & 34 & 19 & 21 \\
\hline 23 & 10 & 18 & 27 & 152 & 41 & 42 & 33 & 16 & 28 \\
\hline 24 & 66 & 12 & 12 & 52 & 18 & 60 & 32 & 13 & 138 \\
\hline Total & 432 & 535 & 1218 & 1488 & 1120 & 1317 & 1253 & 1036 & 1741 \\
\hline
\end{tabular}


Table E.2 Operator I - Tests 2, 3, and 4

\begin{tabular}{|c|c|c|c|c|c|c|c|c|c|}
\cline { 2 - 10 } \multicolumn{1}{c|}{} & \multicolumn{3}{|c|}{ Test 2} & \multicolumn{3}{c|}{ Test 3 } & \multicolumn{3}{c|}{ Test 4} \\
\cline { 2 - 11 } \multicolumn{1}{c|}{} & $\mathrm{J}$ & $\mathrm{M}$ & $\mathrm{P}$ & $\mathrm{K}$ & $\mathrm{N}$ & $\mathrm{Q}$ & $\mathrm{A}$ & $\mathrm{E}$ & $\mathrm{I}$ \\
\hline 1 & 16 & 16 & 41 & 15 & 13 & 20 & 18 & 19 & 16 \\
\hline 2 & 16 & 11 & 36 & 14 & 17 & 17 & 13 & 24 & 26 \\
\hline 3 & 11 & 15 & 21 & 10 & 15 & 20 & 11 & 16 & 18 \\
\hline 4 & 17 & 29 & 28 & 14 & 22 & 28 & 14 & 23 & 28 \\
\hline 5 & 24 & 46 & 19 & 18 & 18 & 36 & 15 & 13 & 36 \\
\hline 6 & 19 & 12 & 26 & 16 & 55 & 91 & 32 & 16 & 13 \\
\hline 7 & 29 & 17 & 14 & 38 & 22 & 27 & 16 & 25 & 19 \\
\hline 8 & 13 & 16 & 53 & 18 & 13 & 31 & 11 & 12 & 47 \\
\hline 9 & 14 & 22 & 37 & 14 & 15 & 17 & 10 & 20 & 16 \\
\hline 10 & 24 & 37 & 93 & 15 & 12 & 50 & 11 & 17 & 17 \\
\hline 11 & 28 & 18 & 105 & 13 & 13 & 16 & 15 & 13 & 53 \\
\hline 12 & 24 & 21 & 65 & 11 & 10 & 23 & 18 & 11 & 144 \\
\hline 13 & 12 & 25 & 88 & 13 & 12 & 40 & 24 & 20 & 19 \\
\hline 14 & 13 & 23 & 27 & 13 & 11 & 21 & 11 & 19 & 29 \\
\hline 15 & 18 & 26 & 52 & 47 & 16 & 22 & 12 & 11 & 33 \\
\hline 16 & 25 & 38 & 47 & 16 & 12 & 48 & 12 & 15 & 49 \\
\hline 17 & 18 & 34 & 47 & 22 & 18 & 30 & 13 & 13 & 28 \\
\hline 18 & 27 & 17 & 69 & 16 & 18 & 69 & 13 & 12 & 20 \\
\hline 19 & 22 & 26 & 102 & 18 & 14 & 70 & 14 & 14 & 22 \\
\hline 20 & 42 & 20 & 32 & 25 & 15 & 36 & 11 & 15 & 30 \\
\hline 21 & 19 & 20 & 19 & 20 & 11 & 37 & 14 & 10 & 17 \\
\hline 22 & 25 & 22 & 32 & 39 & 9 & 32 & 16 & 12 & 10 \\
\hline 23 & 18 & 12 & 25 & 22 & 16 & 12 & 12 & 21 & 14 \\
\hline 24 & 18 & 10 & 30 & 23 & 10 & 14 & 11 & 16 & 23 \\
\hline Total & 492 & 533 & 1108 & 470 & 387 & 807 & 347 & 387 & 727 \\
\hline
\end{tabular}


Table E.3 Operator I - Tests 5, 6, and 7

\begin{tabular}{|c|c|c|c|c|c|c|c|c|c|}
\cline { 2 - 10 } \multicolumn{1}{c|}{} & \multicolumn{3}{|c|}{ Test 5} & \multicolumn{3}{c|}{ Test 6} & \multicolumn{3}{c|}{ Test 7} \\
\cline { 2 - 11 } \multicolumn{1}{c|}{} & B & F & G & L & O & R & C & D & H \\
\hline 1 & 11 & 12 & 50 & 10 & 17 & 29 & 10 & 25 & 56 \\
\hline 2 & 48 & 19 & 39 & 10 & 11 & 31 & 10 & 22 & 20 \\
\hline 3 & 18 & 17 & 14 & 11 & 32 & 24 & 10 & 16 & 22 \\
\hline 4 & 15 & 13 & 25 & 9 & 15 & 23 & 18 & 29 & 38 \\
\hline 5 & 14 & 13 & 25 & 12 & 12 & 18 & 19 & 31 & 47 \\
\hline 6 & 17 & 16 & 20 & 12 & 21 & 67 & 22 & 28 & 13 \\
\hline 7 & 17 & 16 & 17 & 12 & 14 & 25 & 19 & 35 & 18 \\
\hline 8 & 10 & 10 & 20 & 13 & 14 & 30 & 21 & 29 & 47 \\
\hline 9 & 10 & 10 & 67 & 22 & 14 & 24 & 24 & 27 & 15 \\
\hline 10 & 28 & 19 & 62 & 18 & 17 & 22 & 48 & 26 & 18 \\
\hline 11 & 39 & 18 & 38 & 18 & 18 & 32 & 19 & 37 & 37 \\
\hline 12 & 29 & 14 & 49 & 20 & 16 & 85 & 20 & 27 & 17 \\
\hline 13 & 29 & 18 & 91 & 15 & 16 & 50 & 18 & 36 & 37 \\
\hline 14 & 23 & 15 & 39 & 12 & 33 & 25 & 12 & 27 & 34 \\
\hline 15 & 22 & 10 & 33 & 13 & 21 & 30 & 10 & 20 & 22 \\
\hline 16 & 23 & 31 & 31 & 12 & 13 & 25 & 10 & 24 & 55 \\
\hline 17 & 27 & 13 & 41 & 13 & 33 & 24 & 14 & 37 & 37 \\
\hline 18 & 22 & 26 & 86 & 23 & 21 & 32 & 51 & 18 & 60 \\
\hline 19 & 26 & 13 & 45 & 14 & 14 & 17 & 28 & 32 & 54 \\
\hline 20 & 21 & 22 & 28 & 11 & 14 & 44 & 16 & 24 & 25 \\
\hline 21 & 26 & 21 & 16 & 11 & 18 & 19 & 15 & 33 & 27 \\
\hline 22 & 55 & 14 & 20 & 10 & 23 & 17 & 16 & 15 & 42 \\
\hline 23 & 28 & 12 & 18 & 11 & 13 & 17 & 17 & 10 & 20 \\
\hline 24 & 24 & 10 & 14 & 15 & 10 & 16 & 15 & 11 & 32 \\
\hline Total & 582 & 382 & 888 & 327 & 430 & 726 & 462 & 619 & 793 \\
\hline
\end{tabular}


Table E.4 Operator II - Test 1

\begin{tabular}{|c|r|r|r|r|r|r|r|r|r|}
\cline { 2 - 11 } \multicolumn{1}{c|}{} & \multicolumn{3}{|c|}{$60^{\circ} \mathrm{FOV}$} & \multicolumn{3}{c|}{$30^{\circ} \mathrm{FOV}$} & \multicolumn{3}{c|}{$15^{\circ} \mathrm{FOV}$} \\
\cline { 2 - 11 } \multicolumn{1}{c|}{} & $\mathrm{J}$ & $\mathrm{M}$ & $\mathrm{P}$ & $\mathrm{K}$ & $\mathrm{N}$ & $\mathrm{Q}$ & $\mathrm{L}$ & \multicolumn{1}{c|}{$\mathrm{O}$} & \multicolumn{1}{c|}{$\mathrm{R}$} \\
\hline 1 & 14 & 15 & 18 & 22 & 39 & 40 & 27 & 32 & 25 \\
\hline 2 & 15 & 32 & 88 & 15 & 31 & 49 & 22 & 44 & 68 \\
\hline 3 & 12 & 44 & 13 & 43 & 76 & 38 & 51 & 28 & 61 \\
\hline 4 & 10 & 18 & 53 & 20 & 45 & 38 & 16 & 31 & 26 \\
\hline 5 & 12 & 57 & 12 & 25 & 32 & 203 & 33 & 38 & 41 \\
\hline 6 & 10 & 35 & 97 & 15 & 53 & 52 & 67 & 124 & 19 \\
\hline 7 & 36 & 23 & 20 & 76 & 29 & 35 & 71 & 29 & 47 \\
\hline 8 & 66 & 21 & 75 & 15 & 30 & 52 & 78 & 92 & 88 \\
\hline 9 & 19 & 15 & 239 & 24 & 36 & 74 & 32 & 37 & 43 \\
\hline 10 & 23 & 107 & 77 & 16 & 33 & 23 & 32 & 115 & 77 \\
\hline 11 & 19 & 47 & 51 & 116 & 62 & 16 & 34 & 48 & 87 \\
\hline 12 & 24 & 44 & 69 & 37 & 24 & 93 & 36 & 80 & 115 \\
\hline 13 & 31 & 20 & 55 & 30 & 26 & 185 & 25 & 59 & 98 \\
\hline 14 & 63 & 30 & 54 & 59 & 77 & 27 & 41 & 99 & 94 \\
\hline 15 & 19 & 21 & 56 & 30 & 13 & 43 & 36 & 44 & 86 \\
\hline 16 & 25 & 27 & 38 & 110 & 49 & 32 & 20 & 59 & 47 \\
\hline 17 & 14 & 20 & 259 & 87 & 16 & 63 & 69 & 245 & 347 \\
\hline 18 & 82 & 20 & 132 & 31 & 20 & 59 & 17 & 56 & 130 \\
\hline 19 & 42 & 16 & 87 & 61 & 24 & 43 & 34 & 130 & 220 \\
\hline 20 & 27 & 13 & 66 & 44 & 35 & 63 & 34 & 263 & 133 \\
\hline 21 & 11 & 12 & 85 & 60 & 43 & 57 & 274 & 43 & 113 \\
\hline 22 & 19 & 27 & 34 & 52 & 63 & 30 & 31 & 57 & 97 \\
\hline 23 & 29 & 19 & 51 & 42 & 17 & 29 & 85 & 71 & 160 \\
\hline 24 & 87 & 10 & 34 & 70 & 86 & 19 & 125 & 72 & 61 \\
\hline Total & 709 & 693 & 1763 & 1100 & 959 & 1363 & 1290 & 1896 & 2283 \\
\hline
\end{tabular}


Table E.5 Operator II - Tests 2, 3, and 4

\begin{tabular}{|c|c|c|c|c|c|c|c|c|c|}
\hline & \multicolumn{3}{|c|}{ Test 2} & \multicolumn{3}{|c|}{ Test 3} & \multicolumn{3}{|c|}{ Test 4} \\
\hline & $\mathrm{A}$ & $\mathrm{D}$ & $\mathrm{G}$ & $\mathrm{B}$ & $\mathrm{E}$ & $\mathrm{H}$ & $\mathrm{J}$ & $\mathrm{N}$ & $\mathrm{R}$ \\
\hline 1 & 20 & 16 & 21 & 12 & 25 & 30 & 12 & 20 & 30 \\
\hline 2 & 12 & 12 & 28 & 22 & 25 & 17 & 12 & 13 & 30 \\
\hline 3 & 14 & 19 & 23 & 14 & 50 & 17 & 10 & 14 & 32 \\
\hline 4 & 13 & 55 & 26 & 14 & 36 & 30 & 15 & 22 & 40 \\
\hline 5 & 25 & 34 & 21 & 16 & 23 & 43 & 14 & 16 & 18 \\
\hline 6 & 20 & 22 & 26 & 22 & 16 & 82 & 12 & 23 & 18 \\
\hline 7 & 21 & 25 & 17 & 22 & 22 & 39 & 11 & 23 & 11 \\
\hline 8 & 14 & 18 & 17 & 33 & 23 & 59 & 10 & 17 & 15 \\
\hline 9 & 16 & 25 & 213 & 32 & 24 & 17 & 11 & 17 & 37 \\
\hline 10 & 19 & 33 & 45 & 33 & 13 & 23 & 10 & 19 & 34 \\
\hline 11 & 22 & 35 & 82 & 28 & 18 & 18 & 10 & 16 & 72 \\
\hline 12 & 26 & 22 & 150 & 54 & 15 & 17 & 13 & 12 & 56 \\
\hline 13 & 20 & 125 & 53 & 35 & 15 & 41 & 18 & 10 & 30 \\
\hline 14 & 25 & 32 & 76 & 33 & 80 & 22 & 15 & 12 & 71 \\
\hline 15 & 20 & 21 & 45 & 30 & 31 & 27 & 14 & 17 & 79 \\
\hline 16 & 17 & 27 & 28 & 38 & 31 & 48 & 17 & 46 & 30 \\
\hline 17 & 13 & 23 & 78 & 36 & 30 & 44 & 17 & 16 & 35 \\
\hline 18 & 15 & 32 & 49 & 33 & 30 & 54 & 17 & 22 & 23 \\
\hline 19 & 10 & 25 & 99 & 25 & 40 & 66 & 18 & 12 & 16 \\
\hline 20 & 10 & 20 & 29 & 32 & 28 & 64 & 18 & 15 & 24 \\
\hline 21 & 11 & 17 & 51 & 58 & 45 & 74 & 20 & 14 & 33 \\
\hline 22 & 18 & 22 & 60 & 30 & 49 & 65 & 25 & 14 & 20 \\
\hline 23 & 17 & 16 & 54 & 32 & 26 & 15 & 27 & 9 & 23 \\
\hline 24 & 35 & 24 & 39 & 28 & 36 & 48 & 17 & 15 & 48 \\
\hline Total & 433 & 700 & 1330 & 712 & 731 & 960 & 363 & 414 & 825 \\
\hline
\end{tabular}


Table E.6 Operator II - Tests 5, 6, and 7

\begin{tabular}{|c|c|c|c|c|c|c|c|c|c|}
\cline { 2 - 10 } \multicolumn{1}{c|}{} & \multicolumn{3}{|c|}{ Test 5} & \multicolumn{3}{c|}{ Test 6} & \multicolumn{3}{c|}{ Test 7} \\
\cline { 2 - 11 } \multicolumn{1}{c|}{} & K & O & P & C & F & I & L & M & Q \\
\hline 1 & 11 & 22 & 50 & 13 & 12 & 31 & 20 & 25 & 19 \\
\hline 2 & 10 & 20 & 37 & 27 & 14 & 48 & 17 & 20 & 28 \\
\hline 3 & 12 & 25 & 25 & 30 & 21 & 26 & 17 & 24 & 41 \\
\hline 4 & 13 & 23 & 25 & 21 & 10 & 32 & 53 & 20 & 40 \\
\hline 5 & 11 & 22 & 25 & 17 & 26 & 28 & 28 & 21 & 50 \\
\hline 6 & 12 & 22 & 13 & 27 & 20 & 22 & 20 & 58 & 38 \\
\hline 7 & 10 & 21 & 14 & 17 & 14 & 17 & 23 & 26 & 28 \\
\hline 8 & 11 & 25 & 18 & 16 & 13 & 20 & 45 & 28 & 21 \\
\hline 9 & 16 & 15 & 37 & 25 & 19 & 22 & 33 & 22 & 27 \\
\hline 10 & 18 & 13 & 52 & 22 & 19 & 23 & 37 & 23 & 22 \\
\hline 11 & 18 & 14 & 54 & 19 & 18 & 74 & 19 & 23 & 37 \\
\hline 12 & 21 & 43 & 32 & 19 & 22 & 136 & 32 & 23 & 14 \\
\hline 13 & 25 & 25 & 39 & 16 & 12 & 72 & 22 & 25 & 27 \\
\hline 14 & 26 & 18 & 43 & 23 & 18 & 87 & 14 & 23 & 28 \\
\hline 15 & 29 & 15 & 36 & 27 & 17 & 23 & 26 & 26 & 53 \\
\hline 16 & 30 & 20 & 42 & 16 & 13 & 40 & 53 & 38 & 76 \\
\hline 17 & 20 & 23 & 35 & 16 & 25 & 41 & 25 & 37 & 181 \\
\hline 18 & 19 & 28 & 54 & 16 & 21 & 38 & 24 & 32 & 50 \\
\hline 19 & 25 & 33 & 32 & 13 & 12 & 22 & 23 & 20 & 46 \\
\hline 20 & 21 & 19 & 28 & 15 & 19 & 28 & 22 & 19 & 41 \\
\hline 21 & 30 & 14 & 49 & 17 & 21 & 45 & 26 & 17 & 37 \\
\hline 22 & 80 & 11 & 42 & 16 & 11 & 94 & 16 & 20 & 44 \\
\hline 23 & 37 & 12 & 19 & 19 & 16 & 32 & 19 & 27 & 42 \\
\hline 24 & 31 & 12 & 28 & 19 & 38 & 51 & 38 & 12 & 16 \\
\hline Total & 536 & 495 & 829 & 466 & 431 & 1052 & 652 & 609 & 1006 \\
\hline
\end{tabular}


Table E.7 Operator III - Test 1

\begin{tabular}{|c|r|r|r|r|r|r|r|r|r|}
\cline { 2 - 10 } \multicolumn{1}{c|}{} & \multicolumn{3}{|c|}{$60^{\circ} \mathrm{FOV}$} & \multicolumn{3}{c|}{$30^{\circ} \mathrm{FOV}$} & \multicolumn{3}{c|}{$15^{\circ} \mathrm{FOV}$} \\
\cline { 2 - 11 } & B & E & H & C & F & I & A & D & G \\
\hline 1 & 10 & 29 & 21 & 10 & 54 & 31 & 14 & 10 & 222 \\
\hline 2 & 12 & 78 & 16 & 11 & 40 & 49 & 11 & 10 & 398 \\
\hline 3 & 11 & 39 & 49 & 13 & 53 & 65 & 20 & 185 & 28 \\
\hline 4 & 10 & 29 & 39 & 64 & 45 & 88 & 55 & 153 & 287 \\
\hline 5 & 11 & 40 & 32 & 52 & 61 & 47 & 24 & 33 & 46 \\
\hline 6 & 11 & 34 & 18 & 69 & 47 & 39 & 18 & 30 & 29 \\
\hline 7 & 10 & 28 & 23 & 54 & 35 & 113 & 29 & 46 & 32 \\
\hline 8 & 10 & 54 & 27 & 76 & 44 & 170 & 13 & 19 & 35 \\
\hline 9 & 10 & 14 & 18 & 59 & 47 & 149 & 9 & 26 & 124 \\
\hline 10 & 36 & 11 & 27 & 67 & 69 & 109 & 11 & 31 & 182 \\
\hline 11 & 9 & 11 & 17 & 101 & 45 & 203 & 14 & 116 & 68 \\
\hline 12 & 15 & 12 & 10 & 70 & 50 & 95 & 17 & 28 & 82 \\
\hline 13 & 14 & 110 & 19 & 76 & 86 & 61 & 53 & 24 & 101 \\
\hline 14 & 16 & 33 & 34 & 63 & 99 & 90 & 24 & 20 & 71 \\
\hline 15 & 68 & 17 & 20 & 48 & 47 & 93 & 28 & 21 & 42 \\
\hline 16 & 57 & 12 & 76 & 57 & 43 & 32 & 41 & 37 & 72 \\
\hline 17 & 17 & 18 & 25 & 55 & 59 & 30 & 17 & 37 & 37 \\
\hline 18 & 15 & 42 & 86 & 65 & 61 & 120 & 11 & 158 & 102 \\
\hline 19 & 62 & 17 & 256 & 70 & 40 & 17 & 10 & 32 & 230 \\
\hline 20 & 42 & 29 & 101 & 69 & 46 & 17 & 9 & 34 & 397 \\
\hline 21 & 19 & 30 & 45 & 73 & 53 & 21 & 10 & 21 & 127 \\
\hline 22 & 142 & 28 & 41 & 85 & 29 & 17 & 13 & 62 & 26 \\
\hline 23 & 99 & 20 & 46 & 67 & 62 & 26 & 14 & 9 & 23 \\
\hline 24 & 66 & 38 & 117 & 43 & 31 & 65 & 13 & 10 & 44 \\
\hline Total & 772 & 773 & 1163 & 1417 & 1246 & 1747 & 478 & 1152 & 2805 \\
\hline
\end{tabular}


Table E.8 Operator III - Tests 2, 3, and 4

\begin{tabular}{|c|c|c|c|c|c|c|c|c|c|}
\cline { 2 - 10 } \multicolumn{1}{c|}{} & \multicolumn{3}{|c|}{ Test 2} & \multicolumn{3}{c|}{ Test 3 } & \multicolumn{3}{c|}{ Test 4} \\
\cline { 2 - 11 } \multicolumn{1}{c|}{} & $\mathrm{K}$ & $\mathrm{N}$ & $\mathrm{Q}$ & $\mathrm{L}$ & $\mathrm{O}$ & $\mathrm{R}$ & $\mathrm{B}$ & $\mathrm{F}$ & $\mathrm{G}$ \\
\hline 1 & 14 & 28 & 26 & 10 & 16 & 13 & 19 & 15 & 63 \\
\hline 2 & 30 & 18 & 18 & 10 & 18 & 20 & 36 & 12 & 35 \\
\hline 3 & 33 & 34 & 28 & 33 & 21 & 11 & 26 & 16 & 79 \\
\hline 4 & 34 & 18 & 51 & 28 & 32 & 22 & 12 & 38 & 25 \\
\hline 5 & 20 & 19 & 29 & 102 & 13 & 15 & 21 & 38 & 26 \\
\hline 6 & 29 & 21 & 23 & 44 & 40 & 11 & 58 & 24 & 29 \\
\hline 7 & 20 & 17 & 72 & 12 & 17 & 14 & 12 & 13 & 18 \\
\hline 8 & 19 & 31 & 43 & 17 & 20 & 21 & 21 & 17 & 17 \\
\hline 9 & 13 & 28 & 17 & 21 & 79 & 14 & 16 & 24 & 73 \\
\hline 10 & 19 & 15 & 76 & 22 & 64 & 23 & 26 & 10 & 110 \\
\hline 11 & 36 & 17 & 36 & 20 & 22 & 54 & 20 & 13 & 94 \\
\hline 12 & 28 & 22 & 15 & 15 & 12 & 32 & 20 & 14 & 45 \\
\hline 13 & 68 & 14 & 65 & 58 & 34 & 26 & 25 & 13 & 55 \\
\hline 14 & 32 & 20 & 29 & 10 & 16 & 70 & 17 & 15 & 33 \\
\hline 15 & 20 & 23 & 30 & 10 & 16 & 40 & 19 & 12 & 37 \\
\hline 16 & 67 & 31 & 47 & 11 & 43 & 44 & 26 & 16 & 55 \\
\hline 17 & 27 & 26 & 38 & 12 & 22 & 43 & 18 & 23 & 37 \\
\hline 18 & 23 & 21 & 51 & 33 & 64 & 11 & 19 & 22 & 74 \\
\hline 19 & 24 & 25 & 39 & 15 & 30 & 21 & 34 & 16 & 54 \\
\hline 20 & 29 & 23 & 47 & 97 & 12 & 54 & 32 & 13 & 29 \\
\hline 21 & 33 & 15 & 41 & 21 & 30 & 28 & 36 & 17 & 19 \\
\hline 22 & 46 & 11 & 31 & 16 & 20 & 20 & 30 & 17 & 23 \\
\hline 23 & 40 & 18 & 62 & 9 & 11 & 15 & 40 & 11 & 14 \\
\hline 24 & 36 & 12 & 21 & 9 & 19 & 55 & 36 & 13 & 46 \\
\hline Total & 740 & 507 & 935 & 635 & 671 & 677 & 619 & 422 & 1090 \\
\hline
\end{tabular}


Table E.9 Operator III - Tests 5, 6, and 7

\begin{tabular}{|c|c|c|c|c|c|c|c|c|c|}
\cline { 2 - 10 } \multicolumn{1}{c|}{} & \multicolumn{3}{|c|}{ Test 5} & \multicolumn{3}{c|}{ Test 6} & \multicolumn{3}{c|}{ Test 7} \\
\cline { 2 - 11 } \multicolumn{1}{c|}{} & C & D & H & J & M & P & A & E & I \\
\hline 1 & 10 & 21 & 15 & 11 & 26 & 11 & 16 & 24 & 20 \\
\hline 2 & 10 & 10 & 10 & 9 & 15 & 34 & 19 & 23 & 62 \\
\hline 3 & 10 & 20 & 23 & 43 & 22 & 19 & 21 & 51 & 59 \\
\hline 4 & 13 & 58 & 33 & 16 & 37 & 23 & 20 & 23 & 35 \\
\hline 5 & 21 & 14 & 23 & 12 & 69 & 15 & 12 & 35 & 46 \\
\hline 6 & 46 & 14 & 20 & 11 & 24 & 45 & 21 & 27 & 20 \\
\hline 7 & 17 & 21 & 15 & 10 & 18 & 19 & 24 & 58 & 41 \\
\hline 8 & 44 & 17 & 30 & 10 & 17 & 36 & 15 & 44 & 38 \\
\hline 9 & 41 & 21 & 16 & 11 & 13 & 49 & 24 & 25 & 25 \\
\hline 10 & 54 & 14 & 36 & 10 & 69 & 72 & 26 & 19 & 32 \\
\hline 11 & 19 & 26 & 28 & 14 & 28 & 83 & 21 & 20 & 108 \\
\hline 12 & 48 & 21 & 17 & 10 & 35 & 71 & 28 & 30 & 98 \\
\hline 13 & 24 & 22 & 22 & 9 & 31 & 68 & 56 & 19 & 66 \\
\hline 14 & 20 & 22 & 14 & 12 & 30 & 75 & 22 & 18 & 27 \\
\hline 15 & 17 & 23 & 21 & 10 & 26 & 63 & 19 & 17 & 29 \\
\hline 16 & 15 & 24 & 33 & 18 & 31 & 50 & 30 & 19 & 58 \\
\hline 17 & 14 & 21 & 51 & 16 & 24 & 58 & 35 & 13 & 51 \\
\hline 18 & 15 & 21 & 42 & 11 & 30 & 48 & 11 & 32 & 67 \\
\hline 19 & 24 & 20 & 52 & 57 & 32 & 49 & 24 & 20 & 35 \\
\hline 20 & 10 & 38 & 39 & 15 & 35 & 51 & 26 & 37 & 20 \\
\hline 21 & 12 & 11 & 48 & 14 & 25 & 38 & 17 & 104 & 35 \\
\hline 22 & 10 & 10 & 52 & 16 & 23 & 65 & 26 & 36 & 17 \\
\hline 23 & 25 & 10 & 22 & 9 & 19 & 26 & 50 & 28 & 19 \\
\hline 24 & 23 & 10 & 18 & 16 & 17 & 14 & 24 & 28 & 26 \\
\hline Total & 542 & 489 & 680 & 370 & 696 & 1082 & 587 & 750 & 1034 \\
\hline
\end{tabular}


Table E.10 Operator IV - Test 1

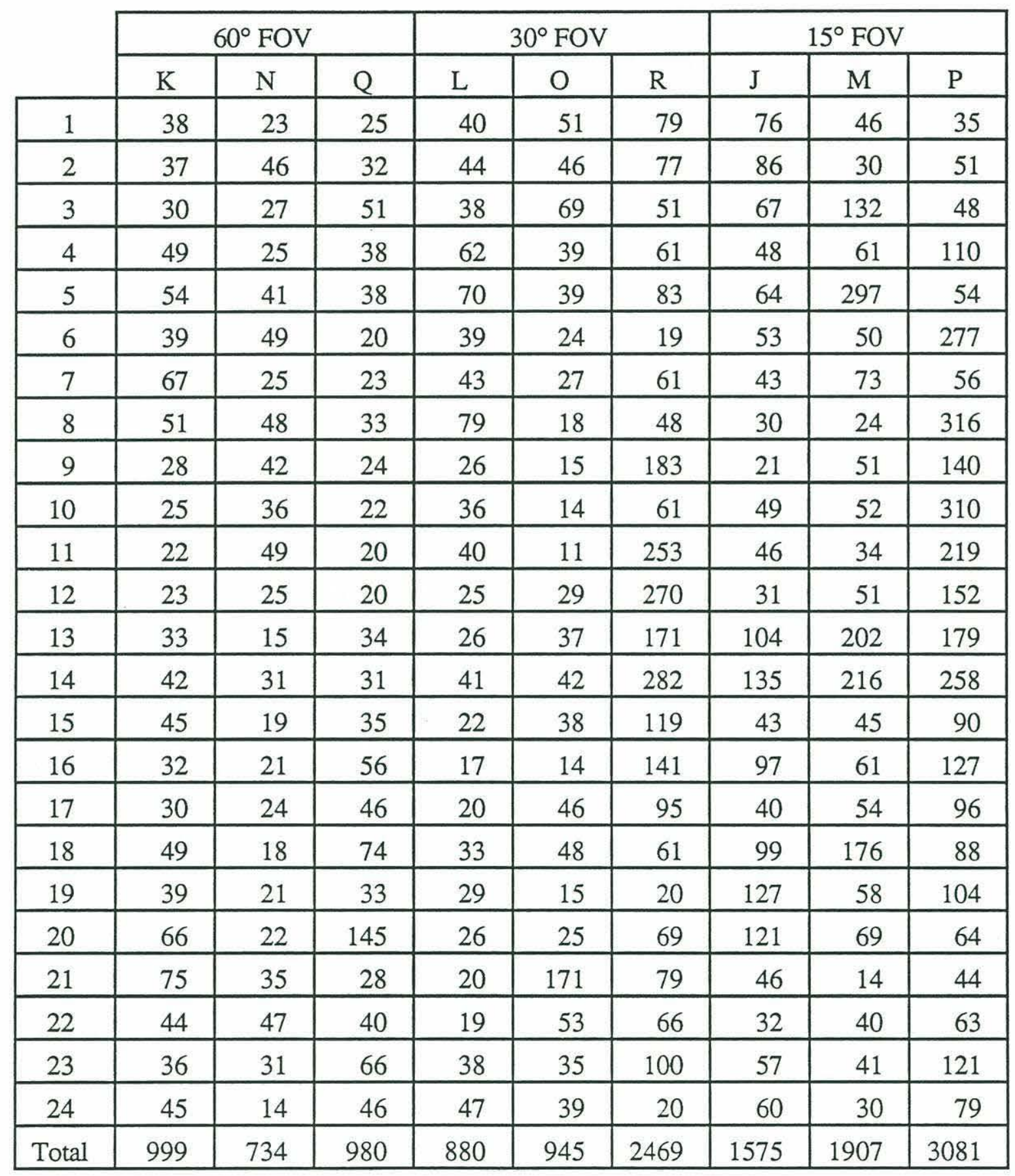


Table E.11 Operator IV - Tests 2, 3, and 4

\begin{tabular}{|c|c|c|c|c|c|c|c|c|c|}
\hline & \multicolumn{3}{|c|}{ Test 2} & \multicolumn{3}{|c|}{ Test 3} & \multicolumn{3}{|c|}{ Test 4} \\
\hline & $\mathrm{B}$ & $\mathrm{E}$ & $\mathrm{H}$ & $\mathrm{C}$ & $\mathrm{F}$ & I & $\mathrm{K}$ & $\mathrm{O}$ & $\mathrm{P}$ \\
\hline 1 & 11 & 17 & 19 & 11 & 15 & 21 & 12 & 11 & 29 \\
\hline 2 & 20 & 22 & 13 & 12 & 38 & 23 & 23 & 17 & 41 \\
\hline 3 & 16 & 21 & 40 & 10 & 21 & 18 & 18 & 24 & 18 \\
\hline 4 & 17 & 27 & 41 & 12 & 16 & 53 & 35 & 26 & 23 \\
\hline 5 & 17 & 37 & 54 & 17 & 12 & 30 & 21 & 22 & 16 \\
\hline 6 & 14 & 21 & 21 & 15 & 16 & 22 & 16 & 17 & 15 \\
\hline 7 & 13 & 17 & 28 & 13 & 19 & 17 & 17 & 13 & 18 \\
\hline 8 & 22 & 22 & 19 & 22 & 19 & 14 & 11 & 30 & 22 \\
\hline 9 & 20 & 19 & 19 & 18 & 18 & 22 & 11 & 18 & 46 \\
\hline 10 & 35 & 22 & 16 & 55 & 16 & 24 & 13 & 10 & 56 \\
\hline 11 & 36 & 26 & 16 & 19 & 18 & 109 & 15 & 43 & 25 \\
\hline 12 & 18 & 13 & 58 & 17 & 31 & 77 & 16 & 16 & 152 \\
\hline 13 & 14 & 10 & 20 & 14 & 16 & 77 & 14 & 14 & 48 \\
\hline 14 & 17 & 16 & 23 & 11 & 21 & 109 & 14 & 17 & 22 \\
\hline 15 & 15 & 12 & 32 & 13 & 36 & 29 & 18 & 18 & 35 \\
\hline 16 & 17 & 16 & 60 & 14 & 27 & 36 & 23 & 13 & 103 \\
\hline 17 & 53 & 12 & 59 & 12 & 16 & 26 & 19 & 16 & 76 \\
\hline 18 & 18 & 20 & 66 & 14 & 11 & 23 & 18 & 13 & 84 \\
\hline 19 & 52 & 18 & 133 & 12 & 14 & 11 & 21 & 11 & 59 \\
\hline 20 & 29 & 18 & 36 & 15 & 11 & 19 & 22 & 13 & 31 \\
\hline 21 & 28 & 20 & 42 & 11 & 11 & 20 & 67 & 14 & 16 \\
\hline 22 & 68 & 17 & 31 & 12 & 18 & 17 & 29 & 14 & 37 \\
\hline 23 & 22 & 11 & 22 & 10 & 15 & 13 & 53 & 10 & 11 \\
\hline 24 & 17 & 11 & 17 & 9 & 10 & 17 & 43 & 10 & 28 \\
\hline Total & 589 & 445 & 885 & 368 & 445 & 827 & 549 & 410 & 1011 \\
\hline
\end{tabular}


Table E.12 Operator IV - Tests 5, 6, and 7

\begin{tabular}{|c|c|c|c|c|c|c|c|c|c|}
\cline { 2 - 10 } \multicolumn{1}{c|}{} & \multicolumn{3}{|c|}{ Test 5} & \multicolumn{3}{c|}{ Test 6} & \multicolumn{3}{c|}{ Test 7} \\
\cline { 2 - 11 } \multicolumn{1}{c|}{} & L & M & Q & A & D & G & J & N & R \\
\hline 1 & 11 & 17 & 17 & 19 & 15 & 25 & 12 & 32 & 12 \\
\hline 2 & 12 & 11 & 11 & 29 & 17 & 23 & 14 & 34 & 35 \\
\hline 3 & 10 & 17 & 20 & 20 & 13 & 19 & 23 & 28 & 71 \\
\hline 4 & 14 & 13 & 24 & 13 & 60 & 28 & 17 & 24 & 24 \\
\hline 5 & 13 & 11 & 21 & 12 & 30 & 16 & 13 & 23 & 18 \\
\hline 6 & 12 & 11 & 16 & 13 & 12 & 15 & 13 & 22 & 40 \\
\hline 7 & 19 & 13 & 58 & 16 & 13 & 18 & 14 & 24 & 25 \\
\hline 8 & 13 & 11 & 16 & 13 & 14 & 24 & 14 & 25 & 29 \\
\hline 9 & 12 & 12 & 15 & 22 & 19 & 45 & 15 & 14 & 28 \\
\hline 10 & 17 & 17 & 38 & 20 & 29 & 84 & 12 & 20 & 23 \\
\hline 11 & 13 & 20 & 14 & 10 & 20 & 43 & 21 & 16 & 60 \\
\hline 12 & 14 & 17 & 15 & 11 & 19 & 61 & 26 & 19 & 61 \\
\hline 13 & 39 & 13 & 17 & 10 & 25 & 58 & 28 & 12 & 53 \\
\hline 14 & 11 & 22 & 19 & 12 & 22 & 99 & 25 & 20 & 172 \\
\hline 15 & 10 & 18 & 124 & 11 & 13 & 51 & 20 & 14 & 42 \\
\hline 16 & 10 & 19 & 32 & 10 & 75 & 40 & 35 & 23 & 44 \\
\hline 17 & 52 & 17 & 75 & 11 & 29 & 92 & 24 & 17 & 31 \\
\hline 18 & 14 & 27 & 91 & 9 & 21 & 72 & 13 & 18 & 22 \\
\hline 19 & 12 & 14 & 45 & 11 & 26 & 73 & 13 & 19 & 11 \\
\hline 20 & 12 & 16 & 52 & 20 & 20 & 23 & 25 & 17 & 26 \\
\hline 21 & 15 & 9 & 35 & 17 & 20 & 16 & 32 & 23 & 26 \\
\hline 22 & 12 & 10 & 25 & 13 & 17 & 22 & 18 & 13 & 19 \\
\hline 23 & 10 & 10 & 14 & 10 & 12 & 15 & 14 & 20 & 24 \\
\hline 24 & 9 & 10 & 23 & 48 & 11 & 28 & 26 & 14 & 18 \\
\hline Total & 366 & 355 & 817 & 380 & 552 & 990 & 467 & 491 & 914 \\
\hline
\end{tabular}




\section{APPENDIX F}

\section{Normalized Data}

Tables F.1 through F.12 list the data for each of the four operators over all seven tests. The tabulated values are normalized values from the associated tables in Appendix E. The data were normalized to remove effects properly attributed to variations in the level of course difficulty related to the Jason vehicle simulation model. The total, mean value, and standard deviation $(\sigma)$ for each column are indicated at the bottom of the column. For all tests the course letter is specified at the top of its respective column. For Test 1 the operator's field of view is also shown.

The normalization procedure divided each time difference by the best time between the associated targets from Appendix D to arrive at the normalized value (dimensionless). Because the logged times represented the most recently passed whole second, while the

best times between targets were calculated to a greater degree of precision, it was possible to achieve a normalized value of 0.9 . 
Table F.1 Operator I - Test 1

\begin{tabular}{|c|r|r|r|r|r|r|r|r|r|}
\cline { 2 - 11 } \multicolumn{1}{c|}{} & \multicolumn{3}{|c|}{$60^{\circ}$ FOV } & \multicolumn{3}{c|}{$30^{\circ}$ FOV } & \multicolumn{3}{c|}{$15^{\circ} \mathrm{FOV}$} \\
\cline { 2 - 11 } \multicolumn{1}{c|}{} & \multicolumn{1}{c|}{ A } & \multicolumn{1}{c|}{ D } & \multicolumn{1}{c|}{ G } & \multicolumn{1}{c|}{ B } & \multicolumn{1}{c|}{ E } & \multicolumn{1}{c|}{ H } & \multicolumn{1}{c|}{ C } & \multicolumn{1}{c|}{ F } & \multicolumn{1}{c|}{ I } \\
\hline 1 & 1.6 & 1.6 & 1.4 & 5.6 & 5.4 & 3.9 & 1.2 & 5.5 & 4.4 \\
\hline 2 & 1.0 & 1.7 & 4.1 & 5.1 & 6.1 & 3.8 & 3.9 & 9.4 & 5.4 \\
\hline 3 & 1.4 & 1.6 & 1.8 & 3.4 & 7.2 & 9.5 & 2.8 & 8.1 & 3.1 \\
\hline 4 & 1.5 & 4.0 & 2.9 & 4.1 & 8.3 & 4.1 & 3.3 & 3.4 & 5.2 \\
\hline 5 & 1.5 & 2.3 & 3.2 & 3.7 & 8.0 & 4.3 & 3.6 & 8.7 & 8.1 \\
\hline 6 & 1.5 & 2.3 & 2.4 & 3.3 & 5.9 & 3.6 & 7.7 & 5.4 & 4.7 \\
\hline 7 & 1.4 & 1.6 & 1.5 & 3.3 & 2.9 & 2.4 & 7.3 & 9.7 & 3.4 \\
\hline 8 & 1.1 & 1.7 & 3.2 & 20.0 & 2.6 & 4.2 & 8.6 & 6.0 & 3.4 \\
\hline 9 & 1.1 & 3.0 & 7.7 & 5.1 & 1.1 & 1.2 & 7.9 & 2.1 & 23.4 \\
\hline 10 & 1.1 & 3.0 & 5.0 & 7.0 & 3.2 & 3.9 & 9.9 & 3.3 & 2.3 \\
\hline 11 & 1.8 & 2.7 & 9.1 & 3.4 & 8.3 & 2.4 & 9.5 & 4.3 & 13.3 \\
\hline 12 & 1.7 & 2.6 & 3.7 & 12.8 & 3.9 & 4.4 & 5.3 & 3.2 & 15.5 \\
\hline 13 & 1.5 & 2.0 & 2.4 & 7.6 & 1.4 & 12.0 & 6.4 & 1.9 & 3.3 \\
\hline 14 & 1.4 & 2.2 & 1.5 & 3.3 & 6.9 & 4.8 & 6.9 & 6.4 & 2.7 \\
\hline 15 & 4.7 & 2.1 & 1.5 & 5.9 & 6.9 & 8.6 & 3.2 & 2.6 & 3.6 \\
\hline 16 & 3.8 & 4.0 & 2.4 & 6.1 & 16.3 & 3.5 & 4.3 & 9.4 & 6.8 \\
\hline 17 & 1.6 & 1.9 & 4.1 & 4.7 & 3.8 & 10.2 & 5.6 & 3.6 & 7.7 \\
\hline 18 & 1.5 & 3.5 & 5.2 & 2.1 & 1.4 & 2.7 & 4.3 & 2.3 & 2.7 \\
\hline 19 & 1.3 & 2.0 & 7.2 & 4.0 & 2.2 & 2.3 & 9.3 & 2.5 & 4.6 \\
\hline 20 & 1.2 & 1.7 & 2.8 & 6.1 & 4.9 & 3.6 & 3.8 & 2.1 & 8.8 \\
\hline 21 & 1.1 & 2.2 & 5.8 & 2.1 & 1.7 & 5.4 & 4.1 & 2.4 & 2.6 \\
\hline 22 & 1.1 & 1.6 & 4.2 & 3.0 & 1.1 & 2.6 & 3.6 & 1.9 & 2.3 \\
\hline 23 & 1.0 & 1.8 & 2.7 & 9.7 & 4.2 & 4.3 & 3.5 & 1.6 & 3.3 \\
\hline 24 & 6.9 & 1.2 & 1.3 & 3.1 & 1.9 & 6.4 & 3.5 & 1.3 & 14.9 \\
\hline Total & 44.0 & 54.3 & 86.9 & 134.4 & 115.6 & 114.0 & 129.3 & 107.5 & 155.3 \\
\hline Mean & 1.8 & 2.3 & 3.6 & 5.6 & 4.8 & 4.8 & 5.4 & 4.5 & 6.5 \\
\hline$\sigma$ & 1.4 & 0.7 & 2.1 & 3.8 & 3.4 & 2.7 & 2.4 & 2.7 & 5.2 \\
\hline & & & & & & & & & \\
\hline
\end{tabular}


Table F.2 Operator I - Tests 2, 3, and 4

\begin{tabular}{|c|r|r|r|r|r|r|r|r|r|}
\cline { 2 - 10 } \multicolumn{1}{c|}{} & \multicolumn{3}{|c|}{ Test 2} & \multicolumn{3}{c|}{ Test 3 } & \multicolumn{3}{c|}{ Test 4} \\
\cline { 2 - 11 } \multicolumn{1}{c|}{} & \multicolumn{1}{c|}{ J } & \multicolumn{1}{c|}{ M } & \multicolumn{1}{c|}{ P } & \multicolumn{1}{c|}{ K } & \multicolumn{1}{c|}{ N } & \multicolumn{1}{c|}{ Q } & \multicolumn{1}{c|}{ A } & \multicolumn{1}{c|}{ E } & \multicolumn{1}{c|}{ I } \\
\hline 1 & 1.6 & 1.6 & 4.3 & 1.5 & 1.3 & 2.2 & 1.8 & 1.9 & 1.7 \\
\hline 2 & 1.6 & 1.1 & 3.8 & 1.4 & 1.8 & 1.7 & 1.3 & 2.5 & 2.9 \\
\hline 3 & 1.1 & 1.6 & 2.3 & 1.0 & 1.6 & 1.9 & 1.1 & 1.7 & 1.9 \\
\hline 4 & 1.7 & 3.1 & 2.9 & 1.4 & 2.3 & 2.0 & 1.4 & 2.5 & 3.2 \\
\hline 5 & 2.4 & 4.8 & 1.9 & 1.8 & 1.8 & 3.2 & 1.5 & 1.3 & 3.7 \\
\hline 6 & 1.9 & 1.2 & 2.8 & 1.6 & 5.9 & 9.5 & 3.2 & 1.7 & 1.4 \\
\hline 7 & 2.9 & 1.8 & 1.5 & 3.8 & 2.4 & 2.8 & 1.6 & 2.7 & 2.0 \\
\hline 8 & 1.3 & 1.6 & 6.1 & 1.9 & 1.4 & 3.4 & 1.1 & 1.3 & 4.8 \\
\hline 9 & 1.4 & 2.4 & 2.2 & 1.5 & 1.6 & 1.8 & 1.0 & 2.1 & 1.7 \\
\hline 10 & 2.4 & 3.2 & 5.3 & 1.6 & 1.2 & 5.4 & 1.1 & 1.7 & 1.5 \\
\hline 11 & 2.8 & 1.7 & 5.3 & 1.4 & 1.3 & 1.7 & 1.5 & 1.3 & 3.7 \\
\hline 12 & 2.4 & 2.3 & 3.6 & 1.2 & 1.0 & 2.5 & 1.8 & 1.1 & 10.1 \\
\hline 13 & 1.2 & 2.7 & 6.1 & 1.3 & 1.2 & 4.5 & 2.4 & 2.0 & 1.3 \\
\hline 14 & 1.3 & 2.5 & 2.2 & 1.1 & 1.1 & 2.3 & 1.1 & 1.9 & 1.7 \\
\hline 15 & 1.8 & 2.9 & 3.2 & 3.8 & 1.6 & 2.0 & 1.2 & 1.1 & 2.0 \\
\hline 16 & 2.6 & 3.1 & 2.5 & 1.3 & 1.2 & 3.2 & 1.3 & 1.6 & 3.1 \\
\hline 17 & 1.8 & 3.1 & 2.5 & 2.1 & 1.8 & 1.6 & 1.3 & 1.3 & 1.9 \\
\hline 18 & 2.7 & 1.9 & 3.9 & 1.4 & 1.8 & 3.9 & 1.3 & 1.2 & 2.2 \\
\hline 19 & 2.2 & 2.9 & 7.0 & 1.4 & 1.4 & 3.5 & 1.4 & 1.4 & 2.4 \\
\hline 20 & 4.2 & 2.2 & 2.4 & 1.8 & 1.6 & 2.1 & 1.1 & 1.6 & 3.2 \\
\hline 21 & 1.9 & 2.1 & 2.1 & 1.4 & 1.1 & 3.5 & 1.4 & 1.0 & 2.0 \\
\hline 22 & 2.5 & 2.3 & 3.5 & 2.5 & 0.9 & 3.2 & 1.6 & 1.2 & 1.1 \\
\hline 23 & 1.9 & 1.2 & 2.5 & 1.4 & 1.6 & 1.2 & 1.2 & 2.1 & 1.7 \\
\hline 24 & 1.9 & 1.0 & 3.3 & 1.4 & 1.0 & 1.5 & 1.1 & 1.6 & 2.5 \\
\hline Total & 49.8 & 54.3 & 83.3 & 41.1 & 40.1 & 70.7 & 35.1 & 40.0 & 63.7 \\
\hline Mean & 2.1 & 2.3 & 3.5 & 1.7 & 1.7 & 2.9 & 1.5 & 1.7 & 2.7 \\
\hline$\sigma$ & 0.7 & 0.9 & 1.5 & 0.7 & 1.0 & 1.7 & 0.5 & 0.5 & 1.8 \\
\hline & & & & & & & & & \\
\hline 19
\end{tabular}


Table F.3 Operator I - Tests 5, 6, and 7

\begin{tabular}{|c|r|r|r|r|r|r|r|r|r|}
\cline { 2 - 11 } \multicolumn{1}{c|}{} & \multicolumn{3}{|c|}{ Test 5 } & \multicolumn{3}{c|}{ Test 6 } & \multicolumn{3}{c|}{ Test 7} \\
\cline { 2 - 12 } \multicolumn{1}{c|}{} & B & F & \multicolumn{1}{c|}{ G } & \multicolumn{1}{c|}{ L } & \multicolumn{1}{c|}{ O } & \multicolumn{1}{c|}{ R } & \multicolumn{1}{c|}{ C } & D & H \\
\hline 1 & 1.1 & 1.2 & 5.2 & 1.0 & 1.8 & 3.0 & 1.0 & 2.5 & 6.2 \\
\hline 2 & 4.9 & 2.0 & 4.1 & 1.0 & 1.1 & 3.4 & 1.0 & 2.2 & 2.0 \\
\hline 3 & 1.8 & 1.9 & 1.6 & 1.1 & 3.5 & 2.5 & 1.0 & 1.7 & 2.1 \\
\hline 4 & 1.5 & 1.4 & 2.6 & 0.9 & 1.6 & 2.7 & 1.8 & 3.1 & 2.7 \\
\hline 5 & 1.4 & 1.4 & 2.5 & 1.2 & 1.3 & 1.8 & 2.0 & 3.2 & 4.2 \\
\hline 6 & 1.7 & 1.7 & 2.1 & 1.2 & 2.3 & 7.4 & 2.2 & 2.9 & 1.4 \\
\hline 7 & 1.7 & 1.6 & 1.8 & 1.2 & 1.4 & 2.7 & 1.9 & 3.6 & 1.9 \\
\hline 8 & 1.0 & 1.0 & 2.3 & 1.3 & 1.4 & 3.1 & 2.2 & 3.0 & 5.1 \\
\hline 9 & 1.1 & 1.0 & 4.0 & 2.3 & 1.4 & 2.6 & 2.5 & 3.0 & 1.6 \\
\hline 10 & 3.0 & 1.9 & 3.5 & 1.9 & 1.7 & 1.9 & 5.1 & 2.2 & 1.9 \\
\hline 11 & 4.2 & 1.8 & 1.9 & 1.9 & 1.8 & 2.2 & 2.0 & 3.5 & 4.0 \\
\hline 12 & 3.2 & 1.4 & 2.7 & 2.1 & 1.6 & 6.0 & 2.1 & 2.9 & 1.8 \\
\hline 13 & 3.0 & 1.8 & 6.3 & 1.5 & 1.6 & 3.5 & 1.8 & 3.9 & 4.2 \\
\hline 14 & 2.0 & 1.6 & 3.2 & 1.2 & 3.5 & 1.5 & 1.2 & 3.0 & 3.7 \\
\hline 15 & 1.8 & 1.0 & 2.0 & 1.3 & 2.1 & 1.8 & 1.0 & 2.2 & 2.0 \\
\hline 16 & 1.8 & 3.1 & 1.7 & 1.2 & 1.3 & 1.6 & 1.0 & 2.0 & 3.7 \\
\hline 17 & 2.5 & 1.4 & 2.1 & 1.3 & 3.6 & 1.6 & 1.4 & 3.3 & 1.9 \\
\hline 18 & 1.9 & 2.7 & 4.8 & 2.4 & 2.2 & 3.5 & 5.2 & 2.0 & 3.4 \\
\hline 19 & 2.1 & 1.3 & 3.1 & 1.5 & 1.4 & 1.9 & 2.9 & 3.6 & 2.7 \\
\hline 20 & 1.5 & 2.2 & 2.1 & 1.2 & 1.4 & 4.7 & 1.7 & 2.6 & 1.5 \\
\hline 21 & 1.8 & 2.1 & 1.8 & 1.1 & 1.8 & 2.2 & 1.5 & 3.4 & 2.6 \\
\hline 22 & 3.5 & 1.4 & 2.2 & 1.1 & 2.3 & 1.9 & 1.7 & 1.5 & 4.1 \\
\hline 23 & 1.8 & 1.2 & 1.8 & 1.2 & 1.3 & 2.0 & 1.8 & 1.0 & 2.0 \\
\hline 24 & 1.4 & 1.0 & 1.6 & 1.6 & 1.0 & 1.7 & 1.6 & 1.1 & 3.4 \\
\hline Total & 51.9 & 39.5 & 67.1 & 33.7 & 44.7 & 67.1 & 47.8 & 63.6 & 70.2 \\
\hline Mean & 2.2 & 1.6 & 2.8 & 1.4 & 1.9 & 2.8 & 2.0 & 2.7 & 2.9 \\
\hline$\sigma$ & 1.0 & 0.5 & 1.2 & 0.4 & 0.7 & 1.4 & 1.1 & 0.8 & 1.2 \\
\hline
\end{tabular}


Table F.4 Operator II - Test 1

\begin{tabular}{|c|c|c|c|c|c|c|c|c|c|}
\hline & \multicolumn{3}{|c|}{$60^{\circ} \mathrm{FOV}$} & \multicolumn{3}{|c|}{$30^{\circ} \mathrm{FOV}$} & \multicolumn{3}{|c|}{$15^{\circ} \mathrm{FOV}$} \\
\hline & $\mathrm{J}$ & $\mathrm{M}$ & $\mathrm{P}$ & $\mathrm{K}$ & $\mathrm{N}$ & $\mathrm{Q}$ & $\mathrm{L}$ & $\mathrm{O}$ & $\mathrm{R}$ \\
\hline 1 & 1.4 & 1.5 & 1.9 & 2.2 & 4.0 & 4.4 & 2.7 & 3.3 & 2.6 \\
\hline 2 & 1.5 & 3.3 & 9.3 & 1.5 & 3.2 & 4.9 & 2.2 & 4.6 & 7.5 \\
\hline 3 & 1.2 & 4.6 & 1.5 & 4.4 & 8.2 & 3.6 & 5.1 & 3.1 & 6.5 \\
\hline 4 & 1.0 & 1.9 & 5.5 & 2.0 & 4.8 & 2.7 & 1.6 & 3.4 & 3.0 \\
\hline 5 & 1.2 & 5.9 & 1.2 & 2.5 & 3.2 & 18.3 & 3.4 & 4.0 & 4.2 \\
\hline 6 & 1.0 & 3.6 & 10.4 & 1.5 & 5.7 & 5.4 & 6.8 & 13.5 & 2.1 \\
\hline 7 & 3.6 & 2.4 & 2.1 & 7.7 & 3.1 & 3.7 & 7.2 & 2.9 & 5.0 \\
\hline 8 & 6.6 & 2.1 & 8.7 & 1.5 & 3.1 & 5.6 & 8.0 & 9.2 & 9.1 \\
\hline 9 & 1.9 & 1.7 & 14.3 & 2.5 & 3.7 & 7.9 & 3.3 & 3.7 & 4.6 \\
\hline 10 & 2.3 & 9.2 & 4.4 & 1.7 & 3.3 & 2.5 & 3.4 & 11.6 & 6.6 \\
\hline 11 & 1.9 & 4.4 & 2.6 & 12.6 & 6.2 & 1.7 & 3.6 & 4.8 & 6.1 \\
\hline 12 & 2.4 & 4.7 & 3.8 & 4.1 & 2.5 & 10.0 & 3.7 & 8.2 & 8.1 \\
\hline 13 & 3.1 & 2.2 & 3.8 & 3.1 & 2.6 & 21.0 & 2.5 & 6.0 & 7.0 \\
\hline 14 & 6.5 & 3.3 & 4.4 & 5.0 & 7.8 & 2.9 & 4.1 & 10.4 & 5.5 \\
\hline 15 & 1.9 & 2.3 & 3.4 & 2.4 & 1.3 & 3.9 & 3.6 & 4.4 & 5.2 \\
\hline 16 & 2.6 & 2.2 & 2.1 & 8.7 & 5.1 & 2.1 & 2.0 & 6.0 & 2.9 \\
\hline 17 & 1.4 & 1.8 & 13.5 & 8.2 & 1.6 & 3.3 & 6.9 & 27.0 & 23.5 \\
\hline 18 & 8.3 & 2.3 & 7.4 & 2.7 & 2.0 & 3.3 & 1.7 & 5.9 & 14.1 \\
\hline 19 & 4.2 & 1.8 & 6.0 & 4.8 & 2.5 & 2.2 & 3.5 & 13.2 & 24.5 \\
\hline 20 & 2.7 & 1.4 & 4.9 & 3.1 & 3.6 & 3.7 & 3.7 & 26.4 & 14.1 \\
\hline 21 & 1.1 & 1.2 & 9.6 & 4.2 & 4.4 & 5.4 & 28.3 & 4.4 & 13.4 \\
\hline 22 & 1.9 & 2.8 & 3.7 & 3.3 & 6.3 & 3.0 & 3.3 & 5.8 & 10.7 \\
\hline 23 & 3.0 & 1.9 & 5.1 & 2.7 & 1.7 & 2.9 & 9.0 & 7.2 & 18.9 \\
\hline 24 & 9.1 & 1.0 & 3.8 & 4.2 & 8.8 & 2.0 & 13.5 & 7.3 & 6.6 \\
\hline Total & 72.1 & 69.7 & 133.3 & 96.9 & 99.0 & 126.6 & 133.4 & 196.4 & 211.6 \\
\hline Mean & 3.0 & 2.9 & 5.6 & 4.0 & 4.1 & 5.3 & 5.6 & 8.2 & 8.8 \\
\hline$\sigma$ & 2.3 & 1.8 & 3.6 & 2.7 & 2.1 & 4.7 & 5.5 & 6.4 & 6.1 \\
\hline
\end{tabular}


Table F.5 Operator II - Tests 2, 3, and 4

\begin{tabular}{|c|c|c|c|c|c|c|c|c|c|}
\hline & \multicolumn{3}{|c|}{ Test 2} & \multicolumn{3}{|c|}{ Test 3} & \multicolumn{3}{|c|}{ Test 4} \\
\hline & A & D & $\mathrm{G}$ & B & E & $\mathrm{H}$ & $\mathrm{J}$ & $\mathrm{N}$ & $\mathrm{R}$ \\
\hline 1 & 2.0 & 1.6 & 2.2 & 1.2 & 2.6 & 3.3 & 1.2 & 2.1 & 3.1 \\
\hline 2 & 1.2 & 1.2 & 2.9 & 2.2 & 2.6 & 1.7 & 1.2 & 1.3 & 3.3 \\
\hline 3 & 1.4 & 2.0 & 2.6 & 1.4 & 5.4 & 1.6 & 1.0 & 1.5 & 3.4 \\
\hline 4 & 1.3 & 5.9 & 2.7 & 1.4 & 3.8 & 2.1 & 1.5 & 2.3 & 4.6 \\
\hline 5 & 2.5 & 3.5 & 2.1 & 1.6 & 2.3 & 3.9 & 1.4 & 1.6 & 1.8 \\
\hline 6 & 2.0 & 2.3 & 2.8 & 2.2 & 1.7 & 8.6 & 1.2 & 2.5 & 2.0 \\
\hline 7 & 2.1 & 2.6 & 1.8 & 2.2 & 2.4 & 4.1 & 1.1 & 2.5 & 1.2 \\
\hline 8 & 1.4 & 1.8 & 2.0 & 3.4 & 2.4 & 6.4 & 1.0 & 1.8 & 1.5 \\
\hline 9 & 1.6 & 2.8 & 12.7 & 3.4 & 2.5 & 1.8 & 1.1 & 1.8 & 3.9 \\
\hline 10 & 1.9 & 2.9 & 2.6 & 3.6 & 1.3 & 2.5 & 1.0 & 1.9 & 2.9 \\
\hline 11 & 2.2 & 3.3 & 4.1 & 3.0 & 1.8 & 2.0 & 1.0 & 1.6 & 5.1 \\
\hline 12 & 2.6 & 2.4 & 8.3 & 6.0 & 1.6 & 1.8 & 1.3 & 1.3 & 3.9 \\
\hline 13 & 2.0 & 13.6 & 3.7 & 3.6 & 1.5 & 4.6 & 1.8 & 1.0 & 2.1 \\
\hline 14 & 2.6 & 3.5 & 6.2 & 2.8 & 8.1 & 2.4 & 1.5 & 1.2 & 4.1 \\
\hline 15 & 2.0 & 2.3 & 2.8 & 2.4 & 3.1 & 2.5 & 1.4 & 1.7 & 4.8 \\
\hline 16 & 1.8 & 2.2 & 1.5 & 3.0 & 3.2 & 3.2 & 1.8 & 4.8 & 1.9 \\
\hline 17 & 1.3 & 2.1 & 4.1 & 3.4 & 3.0 & 2.3 & 1.7 & 1.6 & 2.4 \\
\hline 18 & 1.5 & 3.6 & 2.7 & 2.8 & 3.0 & 3.0 & 1.7 & 2.2 & 2.5 \\
\hline 19 & 1.0 & 2.8 & 6.8 & 2.0 & 4.1 & 3.3 & 1.8 & 1.2 & 1.8 \\
\hline 20 & 1.0 & 2.2 & 2.1 & 2.3 & 2.9 & 3.8 & 1.8 & 1.6 & 2.5 \\
\hline 21 & 1.1 & 1.8 & 5.8 & 4.1 & 4.6 & 7.1 & 2.0 & 1.4 & 3.9 \\
\hline 22 & 1.8 & 2.3 & 6.6 & 1.9 & 4.9 & 6.4 & 2.5 & 1.4 & 2.2 \\
\hline 23 & 1.8 & 1.6 & 5.4 & 2.0 & 2.7 & 1.5 & 2.8 & 0.9 & 2.7 \\
\hline 24 & 3.7 & 2.4 & 4.4 & 1.7 & 3.7 & 5.1 & 1.8 & 1.5 & 5.2 \\
\hline Total & 43.9 & 72.6 & 98.9 & 63.8 & 75.3 & 85.0 & 36.8 & 42.7 & 72.9 \\
\hline Mean & 1.8 & 3.0 & 4.1 & 2.7 & 3.1 & 3.5 & 1.5 & 1.8 & 3.0 \\
\hline$\sigma$ & 0.6 & 2.4 & 2.6 & 1.0 & 1.5 & 1.9 & 0.5 & 0.8 & 1.2 \\
\hline
\end{tabular}


Table F.6 Operator II - Tests 5, 6, and 7

\begin{tabular}{|c|r|r|r|r|r|r|r|r|r|}
\cline { 2 - 11 } \multicolumn{1}{c|}{} & \multicolumn{3}{|c|}{ Test 5 } & \multicolumn{3}{c|}{ Test 6 } & \multicolumn{3}{c|}{ Test 7 } \\
\cline { 2 - 11 } \multicolumn{1}{c|}{} & \multicolumn{1}{c|}{ K } & \multicolumn{1}{c|}{ O } & \multicolumn{1}{c|}{ P } & \multicolumn{1}{c|}{ F } & \multicolumn{1}{c|}{ I } & \multicolumn{1}{c|}{ L } & M & Q \\
\hline 1 & 1.1 & 2.3 & 5.2 & 1.3 & 1.2 & 3.2 & 2.0 & 2.5 & 2.1 \\
\hline 2 & 1.0 & 2.1 & 3.9 & 2.7 & 1.5 & 5.3 & 1.7 & 2.0 & 2.8 \\
\hline 3 & 1.2 & 2.7 & 2.8 & 3.0 & 2.3 & 2.8 & 1.7 & 2.5 & 3.8 \\
\hline 4 & 1.3 & 2.5 & 2.6 & 2.1 & 1.1 & 3.7 & 5.4 & 2.2 & 2.8 \\
\hline 5 & 1.1 & 2.3 & 2.5 & 1.8 & 2.8 & 2.9 & 2.9 & 2.2 & 4.5 \\
\hline 6 & 1.2 & 2.4 & 1.4 & 2.8 & 2.2 & 2.4 & 2.0 & 6.0 & 4.0 \\
\hline 7 & 1.0 & 2.1 & 1.5 & 1.7 & 1.4 & 1.8 & 2.3 & 2.7 & 2.9 \\
\hline 8 & 1.1 & 2.5 & 2.1 & 1.7 & 1.3 & 2.1 & 4.6 & 2.9 & 2.3 \\
\hline 9 & 1.7 & 1.5 & 2.2 & 2.6 & 1.9 & 2.3 & 3.4 & 2.4 & 2.9 \\
\hline 10 & 2.0 & 1.3 & 3.0 & 2.4 & 1.9 & 2.0 & 4.0 & 2.0 & 2.4 \\
\hline 11 & 2.0 & 1.4 & 2.7 & 2.0 & 1.8 & 5.2 & 2.0 & 2.2 & 4.0 \\
\hline 12 & 2.3 & 4.4 & 1.8 & 2.0 & 2.3 & 9.5 & 3.3 & 2.5 & 1.5 \\
\hline 13 & 2.5 & 2.5 & 2.7 & 1.6 & 1.2 & 5.1 & 2.2 & 2.7 & 3.1 \\
\hline 14 & 2.2 & 1.9 & 3.5 & 2.3 & 1.9 & 5.1 & 1.4 & 2.5 & 3.0 \\
\hline 15 & 2.4 & 1.5 & 2.2 & 2.7 & 1.7 & 1.4 & 2.6 & 2.9 & 4.9 \\
\hline 16 & 2.4 & 2.0 & 2.3 & 1.6 & 1.3 & 2.5 & 5.3 & 3.1 & 5.1 \\
\hline 17 & 1.9 & 2.5 & 1.8 & 1.6 & 2.8 & 2.8 & 2.5 & 3.3 & 9.5 \\
\hline 18 & 1.6 & 3.0 & 3.0 & 1.6 & 2.2 & 4.1 & 2.5 & 3.6 & 2.8 \\
\hline 19 & 2.0 & 3.4 & 2.2 & 1.4 & 1.2 & 2.4 & 2.4 & 2.2 & 2.3 \\
\hline 20 & 1.5 & 1.9 & 2.1 & 1.6 & 1.9 & 3.0 & 2.4 & 2.1 & 2.4 \\
\hline 21 & 2.1 & 1.4 & 5.5 & 1.8 & 2.1 & 5.3 & 2.7 & 1.8 & 3.5 \\
\hline 22 & 5.1 & 1.1 & 4.6 & 1.7 & 1.1 & 10.4 & 1.7 & 2.1 & 4.3 \\
\hline 23 & 2.4 & 1.2 & 1.9 & 2.0 & 1.6 & 3.8 & 2.0 & 2.7 & 4.3 \\
\hline 24 & 1.9 & 1.2 & 3.1 & 2.1 & 3.9 & 5.5 & 4.1 & 1.2 & 1.7 \\
\hline Total & 45.0 & 51.3 & 66.7 & 47.9 & 44.6 & 94.5 & 67.2 & 62.3 & 82.9 \\
\hline Mean & 1.9 & 2.1 & 2.8 & 2.0 & 1.9 & 3.9 & 2.8 & 2.6 & 3.5 \\
\hline$\sigma$ & 0.8 & 0.8 & 1.1 & 0.5 & 0.6 & 2.2 & 1.1 & 0.9 & 1.6 \\
\hline
\end{tabular}


Table F.7 Operator III - Test 1

\begin{tabular}{|c|c|c|c|c|c|c|c|c|c|}
\hline & \multicolumn{3}{|c|}{$60^{\circ} \mathrm{FOV}$} & \multicolumn{3}{|c|}{$30^{\circ} \mathrm{FOV}$} & \multicolumn{3}{|c|}{$15^{\circ} \mathrm{FOV}$} \\
\hline & $\mathrm{B}$ & $\mathrm{E}$ & $\mathrm{H}$ & $\mathrm{C}$ & $\mathrm{F}$ & I & A & $\mathrm{D}$ & G \\
\hline 1 & 1.0 & 3.0 & 2.3 & 1.0 & 5.6 & 3.2 & 1.4 & 1.0 & 23.2 \\
\hline 2 & 1.2 & 8.1 & 1.6 & 1.1 & 4.2 & 5.4 & 1.1 & 1.0 & 41.9 \\
\hline 3 & 1.1 & 4.2 & 4.6 & 1.3 & 5.8 & 6.9 & 2.0 & 19.4 & 3.1 \\
\hline 4 & 1.0 & 3.1 & 2.7 & 6.5 & 4.9 & 10.2 & 5.5 & 16.5 & 29.9 \\
\hline 5 & 1.1 & 4.0 & 2.9 & 5.4 & 6.5 & 4.8 & 2.4 & 3.4 & 4.6 \\
\hline 6 & 1.1 & 3.6 & 1.9 & 7.1 & 5.1 & 4.3 & 1.8 & 3.1 & 3.1 \\
\hline 7 & 1.0 & 3.0 & 2.4 & 5.5 & 3.5 & 12.1 & 2.9 & 4.8 & 3.4 \\
\hline 8 & 1.0 & 5.6 & 2.9 & 7.8 & 4.4 & 17.5 & 1.3 & 1.9 & 4.1 \\
\hline 9 & 1.1 & 1.4 & 1.9 & 6.1 & 4.7 & 15.9 & 0.9 & 2.9 & 7.4 \\
\hline 10 . & 3.9 & 1.1 & 2.9 & 7.2 & 7.0 & 9.4 & 1.1 & 2.7 & 10.4 \\
\hline 11 & 1.0 & 1.1 & 1.9 & 10.7 & 4.5 & 14.2 & 1.4 & 10.9 & 3.4 \\
\hline 12 & 1.7 & 1.3 & 1.1 & 7.2 & 5.1 & 6.7 & 1.7 & 3.0 & 4.6 \\
\hline 13 & 1.4 & 11.1 & 2.2 & 7.7 & 8.7 & 4.3 & 5.4 & 2.6 & 7.0 \\
\hline 14 & 1.4 & 3.4 & 3.7 & 6.3 & 10.4 & 5.2 & 2.5 & 2.2 & 5.8 \\
\hline 15 & 5.5 & 1.7 & 1.8 & 4.8 & 4.7 & 5.7 & 2.9 & 2.3 & 2.6 \\
\hline 16 & 4.5 & 1.2 & 5.1 & 5.7 & 4.4 & 2.0 & 4.3 & 3.0 & 3.9 \\
\hline 17 & 1.6 & 1.8 & 1.3 & 5.5 & 6.5 & 2.0 & 1.7 & 3.3 & 1.9 \\
\hline 18 & 1.3 & 4.3 & 4.9 & 6.7 & 6.5 & 13.0 & 1.1 & 17.8 & 5.7 \\
\hline 19 & 4.9 & 1.7 & 12.9 & 7.3 & 4.1 & 1.9 & 1.0 & 3.6 & 15.9 \\
\hline 20 & 3.0 & 3.0 & 6.0 & 7.4 & 4.6 & 1.8 & 0.9 & 3.7 & 29.2 \\
\hline 21 & 1.3 & 3.1 & 4.3 & 7.5 & 5.4 & 2.5 & 1.0 & 2.2 & 14.3 \\
\hline 22 & 9.1 & 2.8 & 4.0 & 8.9 & 3.0 & 1.9 & 1.3 & 6.4 & 2.9 \\
\hline 23 & 6.3 & 2.0 & 4.7 & 7.1 & 6.3 & 3.1 & 1.5 & 0.9 & 2.3 \\
\hline 24 & 4.0 & 3.9 & 12.4 & 4.7 & 3.2 & 7.0 & 1.4 & 1.0 & 4.9 \\
\hline Total & 60.6 & 79.6 & 92.4 & 146.5 & 128.9 & 160.9 & 48.5 & 119.6 & 235.4 \\
\hline Mean & 2.5 & 3.3 & 3.9 & 6.1 & 5.4 & 6.7 & 2.0 & 5.0 & 9.8 \\
\hline$\sigma$ & 2.1 & 2.3 & 3.0 & 2.3 & 1.6 & 4.7 & 1.3 & 5.3 & 10.5 \\
\hline
\end{tabular}


Table F.8 Operator III - Tests 2, 3, and 4

\begin{tabular}{|c|r|r|r|r|r|r|r|r|r|}
\cline { 2 - 10 } \multicolumn{1}{c|}{} & \multicolumn{3}{|c|}{ Test 2} & \multicolumn{3}{c|}{ Test 3 } & \multicolumn{3}{c|}{ Test 4} \\
\cline { 2 - 11 } \multicolumn{1}{c|}{} & \multicolumn{1}{c|}{ K } & \multicolumn{1}{c|}{ N } & \multicolumn{1}{c|}{ Q } & \multicolumn{1}{c|}{ L } & \multicolumn{1}{c|}{ O } & \multicolumn{1}{c|}{ R } & \multicolumn{1}{c|}{ B } & \multicolumn{1}{c|}{ F } & \multicolumn{1}{c|}{ G } \\
\hline 1 & 1.4 & 2.9 & 2.9 & 1.0 & 1.7 & 1.3 & 1.9 & 1.6 & 6.6 \\
\hline 2 & 3.1 & 1.9 & 1.8 & 1.0 & 1.9 & 2.2 & 3.7 & 1.2 & 3.7 \\
\hline 3 & 3.4 & 3.7 & 2.6 & 3.3 & 2.3 & 1.2 & 2.7 & 1.8 & 8.8 \\
\hline 4 & 3.4 & 1.9 & 3.6 & 2.8 & 3.5 & 2.5 & 1.2 & 4.2 & 2.6 \\
\hline 5 & 2.0 & 1.9 & 2.6 & 10.5 & 1.4 & 1.5 & 2.1 & 4.0 & 2.6 \\
\hline 6 & 2.9 & 2.2 & 2.4 & 4.5 & 4.4 & 1.2 & 5.8 & 2.6 & 3.1 \\
\hline 7 & 2.0 & 1.8 & 7.5 & 1.2 & 1.7 & 1.5 & 1.2 & 1.3 & 1.9 \\
\hline 8 & 2.0 & 3.2 & 4.7 & 1.8 & 2.0 & 2.2 & 2.2 & 1.7 & 2.0 \\
\hline 9 & 1.4 & 2.9 & 1.8 & 2.2 & 7.9 & 1.5 & 1.7 & 2.4 & 4.4 \\
\hline 10 & 2.1 & 1.5 & 8.2 & 2.4 & 6.4 & 2.0 & 2.8 & 1.0 & 6.3 \\
\hline 11 & 3.9 & 1.7 & 3.9 & 2.1 & 2.2 & 3.8 & 2.2 & 1.3 & 4.7 \\
\hline 12 & 3.1 & 2.3 & 1.6 & 1.6 & 1.2 & 2.2 & 2.2 & 1.4 & 2.5 \\
\hline 13 & 6.9 & 1.4 & 7.4 & 5.9 & 3.4 & 1.8 & 2.5 & 1.3 & 3.8 \\
\hline 14 & 2.7 & 2.0 & 3.1 & 1.0 & 1.7 & 4.1 & 1.4 & 1.6 & 2.7 \\
\hline 15 & 1.6 & 2.3 & 2.8 & 1.0 & 1.6 & 2.4 & 1.5 & 1.2 & 2.3 \\
\hline 16 & 5.3 & 3.2 & 3.1 & 1.1 & 4.4 & 2.7 & 2.1 & 1.6 & 3.0 \\
\hline 17 & 2.5 & 2.6 & 2.0 & 1.2 & 2.4 & 2.9 & 1.7 & 2.5 & 1.9 \\
\hline 18 & 2.0 & 2.1 & 2.9 & 3.4 & 6.8 & 1.2 & 1.6 & 2.3 & 4.2 \\
\hline 19 & 1.9 & 2.6 & 2.0 & 1.6 & 3.1 & 2.3 & 2.7 & 1.6 & 3.7 \\
\hline 20 & 2.1 & 2.4 & 2.8 & 10.4 & 1.2 & 5.7 & 2.3 & 1.3 & 2.1 \\
\hline 21 & 2.3 & 1.5 & 3.9 & 2.2 & 3.0 & 3.3 & 2.5 & 1.7 & 2.1 \\
\hline 22 & 2.9 & 1.1 & 3.1 & 1.7 & 2.0 & 2.2 & 1.9 & 1.7 & 2.5 \\
\hline 23 & 2.6 & 1.8 & 6.3 & 1.0 & 1.1 & 1.8 & 2.6 & 1.1 & 1.4 \\
\hline 24 & 2.2 & 1.2 & 2.2 & 1.0 & 1.9 & 5.9 & 2.2 & 1.3 & 5.1 \\
\hline Total & 65.7 & 52.3 & 85.1 & 65.6 & 69.3 & 59.6 & 54.8 & 43.9 & 84.1 \\
\hline Mean & 2.7 & 2.2 & 3.5 & 2.7 & 2.9 & 2.5 & 2.3 & 1.8 & 3.5 \\
\hline$\sigma$ & 1.2 & 0.6 & 1.9 & 2.6 & 1.8 & 1.3 & 0.9 & 0.8 & 1.7 \\
\hline & & & & & & & & & \\
\hline
\end{tabular}


Table F.9 Operator III - Tests 5, 6, and 7

\begin{tabular}{|c|r|r|r|r|r|r|r|r|r|}
\cline { 2 - 11 } \multicolumn{1}{c|}{} & \multicolumn{3}{|c|}{ Test 5 } & \multicolumn{3}{c|}{ Test 6 } & \multicolumn{3}{c|}{ Test 7 } \\
\cline { 2 - 12 } \multicolumn{1}{c|}{} & \multicolumn{1}{c|}{ C } & \multicolumn{1}{c|}{ D } & \multicolumn{1}{c|}{ H } & \multicolumn{1}{c|}{ J } & \multicolumn{1}{c|}{ M } & \multicolumn{1}{c|}{ P } & \multicolumn{1}{c|}{ E } & \multicolumn{1}{c|}{ I } \\
\hline 1 & 1.0 & 2.1 & 1.7 & 1.1 & 2.6 & 1.1 & 1.6 & 2.5 & 2.1 \\
\hline 2 & 1.0 & 1.0 & 1.0 & 0.9 & 1.5 & 3.6 & 1.9 & 2.4 & 6.9 \\
\hline 3 & 1.0 & 2.1 & 2.2 & 4.3 & 2.3 & 2.1 & 2.1 & 5.5 & 6.3 \\
\hline 4 & 1.3 & 6.3 & 2.3 & 1.6 & 4.0 & 2.4 & 2.0 & 2.5 & 4.0 \\
\hline 5 & 2.2 & 1.5 & 2.1 & 1.2 & 7.2 & 1.5 & 1.2 & 3.5 & 4.7 \\
\hline 6 & 4.7 & 1.5 & 2.1 & 1.1 & 2.5 & 4.8 & 2.1 & 2.9 & 2.2 \\
\hline 7 & 1.7 & 2.2 & 1.6 & 1.0 & 1.9 & 2.0 & 2.4 & 6.3 & 4.4 \\
\hline 8 & 4.5 & 1.7 & 3.3 & 1.0 & 1.7 & 4.2 & 1.5 & 4.6 & 3.9 \\
\hline 9 & 4.2 & 2.3 & 1.7 & 1.1 & 1.4 & 2.9 & 2.4 & 2.6 & 2.7 \\
\hline 10 & 5.8 & 1.2 & 3.9 & 1.0 & 6.0 & 4.1 & 2.6 & 1.9 & 2.7 \\
\hline 11 & 2.0 & 2.4 & 3.1 & 1.4 & 2.6 & 4.2 & 2.1 & 2.0 & 7.6 \\
\hline 12 & 5.0 & 2.3 & 1.8 & 1.0 & 3.8 & 3.9 & 2.8 & 3.1 & 6.9 \\
\hline 13 & 2.4 & 2.4 & 2.5 & 0.9 & 3.4 & 4.7 & 5.7 & 1.9 & 4.7 \\
\hline 14 & 2.0 & 2.4 & 1.5 & 1.2 & 3.3 & 6.2 & 2.3 & 1.8 & 1.6 \\
\hline 15 & 1.7 & 2.6 & 1.9 & 1.0 & 2.9 & 3.9 & 1.9 & 1.7 & 1.8 \\
\hline 16 & 1.5 & 2.0 & 2.2 & 1.9 & 2.6 & 2.7 & 3.1 & 2.0 & 3.6 \\
\hline 17 & 1.4 & 1.9 & 2.7 & 1.6 & 2.2 & 3.0 & 3.6 & 1.3 & 3.4 \\
\hline 18 & 1.5 & 2.4 & 2.4 & 1.1 & 3.4 & 2.7 & 1.1 & 3.2 & 7.3 \\
\hline 19 & 2.5 & 2.2 & 2.6 & 5.7 & 3.6 & 3.4 & 2.4 & 2.1 & 3.9 \\
\hline 20 & 1.1 & 4.1 & 2.3 & 1.5 & 3.8 & 3.8 & 2.6 & 3.8 & 2.1 \\
\hline 21 & 1.2 & 1.1 & 4.6 & 1.4 & 2.6 & 4.3 & 1.7 & 10.6 & 4.1 \\
\hline 22 & 1.1 & 1.0 & 5.1 & 1.6 & 2.4 & 7.1 & 2.6 & 3.6 & 1.9 \\
\hline 23 & 2.6 & 1.0 & 2.2 & 0.9 & 1.9 & 2.6 & 5.2 & 2.9 & 2.2 \\
\hline 24 & 2.5 & 1.0 & 1.9 & 1.7 & 1.7 & 1.6 & 2.5 & 2.9 & 2.8 \\
\hline Total & 56.0 & 50.7 & 58.6 & 37.5 & 71.1 & 82.8 & 59.6 & 77.6 & 93.7 \\
\hline Mean & 2.3 & 2.1 & 2.4 & 1.6 & 3.0 & 3.5 & 2.5 & 3.2 & 3.9 \\
\hline$\sigma$ & 1.4 & 1.1 & 0.9 & 1.1 & 1.3 & 1.4 & 1.0 & 1.9 & 1.8 \\
\hline
\end{tabular}


Table F.10 Operator IV - Test 1

\begin{tabular}{|c|r|r|r|r|r|r|r|r|r|}
\cline { 2 - 10 } \multicolumn{1}{c|}{} & \multicolumn{3}{|c|}{$60^{\circ} \mathrm{FOV}$} & \multicolumn{3}{c|}{$30^{\circ} \mathrm{FOV}$} & \multicolumn{3}{c|}{$15^{\circ} \mathrm{FOV}$} \\
\cline { 2 - 11 } \multicolumn{1}{c|}{} & $\mathrm{K}$ & \multicolumn{1}{c|}{$\mathrm{N}$} & \multicolumn{1}{c|}{$\mathrm{Q}$} & \multicolumn{1}{c|}{$\mathrm{L}$} & \multicolumn{1}{c|}{$\mathrm{O}$} & \multicolumn{1}{c|}{$\mathrm{R}$} & \multicolumn{1}{c|}{$\mathrm{J}$} & \multicolumn{1}{c|}{$\mathrm{M}$} & \multicolumn{1}{c|}{$\mathrm{P}$} \\
\hline 1 & 3.8 & 2.4 & 2.8 & 4.0 & 5.3 & 8.2 & 7.6 & 4.7 & 3.7 \\
\hline 2 & 3.8 & 4.8 & 3.2 & 4.4 & 4.8 & 8.5 & 8.6 & 3.1 & 5.4 \\
\hline 3 & 3.1 & 2.9 & 4.8 & 3.8 & 7.6 & 5.4 & 6.7 & 13.8 & 5.4 \\
\hline 4 & 5.0 & 2.7 & 2.7 & 6.3 & 4.3 & 7.0 & 4.8 & 6.6 & 11.4 \\
\hline 5 & 5.5 & 4.1 & 3.4 & 7.2 & 4.1 & 8.5 & 6.4 & 30.9 & 5.4 \\
\hline 6 & 3.9 & 5.2 & 2.1 & 4.0 & 2.6 & 2.1 & 5.3 & 5.2 & 29.6 \\
\hline 7 & 6.8 & 2.7 & 2.4 & 4.4 & 2.7 & 6.5 & 4.3 & 7.6 & 5.9 \\
\hline 8 & 5.2 & 5.0 & 3.6 & 8.2 & 1.8 & 4.9 & 3.0 & 2.5 & 36.6 \\
\hline 9 & 2.9 & 4.3 & 2.5 & 2.7 & 1.5 & 19.5 & 2.1 & 5.6 & 8.4 \\
\hline 10 & 2.7 & 3.6 & 2.4 & 3.9 & 1.4 & 5.2 & 4.9 & 4.5 & 17.7 \\
\hline 11 & 2.4 & 4.9 & 2.2 & 4.3 & 1.1 & 17.8 & 4.6 & 3.2 & 11.0 \\
\hline 12 & 2.5 & 2.6 & 2.2 & 2.6 & 3.0 & 18.9 & 3.1 & 5.5 & 8.4 \\
\hline 13 & 3.4 & 1.5 & 3.9 & 2.6 & 3.8 & 12.1 & 10.5 & 22.0 & 12.4 \\
\hline 14 & 3.6 & 3.2 & 3.4 & 4.1 & 4.4 & 16.4 & 13.9 & 23.6 & 21.2 \\
\hline 15 & 3.7 & 1.9 & 3.2 & 2.2 & 3.8 & 7.2 & 4.4 & 5.0 & 5.5 \\
\hline 16 & 2.5 & 2.2 & 3.7 & 1.7 & 1.4 & 8.8 & 10.2 & 5.0 & 6.9 \\
\hline 17 & 2.8 & 2.4 & 2.4 & 2.0 & 5.1 & 6.4 & 4.1 & 4.9 & 5.0 \\
\hline 18 & 4.2 & 1.8 & 4.2 & 3.4 & 5.1 & 6.6 & 10.0 & 19.8 & 4.9 \\
\hline 19 & 3.1 & 2.2 & 1.7 & 3.0 & 1.5 & 2.2 & 12.8 & 6.5 & 7.2 \\
\hline 20 & 4.7 & 2.3 & 8.6 & 2.8 & 2.5 & 7.3 & 12.1 & 7.4 & 4.7 \\
\hline 21 & 5.3 & 3.6 & 2.7 & 2.1 & 17.3 & 9.3 & 4.6 & 1.4 & 5.0 \\
\hline 22 & 2.8 & 4.7 & 3.9 & 2.0 & 5.4 & 7.3 & 3.2 & 4.1 & 6.9 \\
\hline 23 & 2.3 & 3.2 & 6.7 & 4.0 & 3.5 & 11.8 & 5.9 & 4.2 & 12.2 \\
\hline 24 & 2.7 & 1.4 & 4.9 & 5.1 & 4.0 & 2.2 & 6.3 & 3.0 & 8.8 \\
\hline Total & 88.8 & 75.6 & 83.4 & 90.6 & 98.0 & 210.3 & 159.7 & 200.1 & 249.6 \\
\hline Mean & 3.7 & 3.2 & 3.5 & 3.8 & 4.1 & 8.8 & 6.7 & 8.3 & 10.4 \\
\hline$\sigma$ & 1.2 & 1.2 & 1.5 & 1.6 & 3.2 & 4.9 & 3.3 & 7.6 & 8.1 \\
\hline & & & & & & & & & \\
\hline
\end{tabular}


Table F.11 Operator IV - Tests 2, 3, and 4

\begin{tabular}{|c|r|r|r|r|r|r|r|r|r|}
\cline { 2 - 10 } \multicolumn{1}{c|}{} & \multicolumn{3}{|c|}{ Test 2} & \multicolumn{3}{c|}{ Test 3 } & \multicolumn{3}{c|}{ Test 4} \\
\cline { 2 - 11 } \multicolumn{1}{c|}{} & \multicolumn{1}{c|}{ B } & \multicolumn{1}{c|}{ E } & \multicolumn{1}{c|}{ H } & \multicolumn{1}{c|}{ C } & \multicolumn{1}{c|}{ F } & \multicolumn{1}{c|}{ I } & \multicolumn{1}{c|}{ K } & \multicolumn{1}{c|}{ O } & \multicolumn{1}{c|}{ P } \\
\hline 1 & 1.1 & 1.7 & 2.1 & 1.1 & 1.6 & 2.2 & 1.2 & 1.1 & 3.0 \\
\hline 2 & 2.0 & 2.3 & 1.3 & 1.2 & 4.0 & 2.6 & 2.3 & 1.8 & 4.3 \\
\hline 3 & 1.6 & 2.3 & 3.7 & 1.0 & 2.3 & 1.9 & 1.8 & 2.6 & 2.0 \\
\hline 4 & 1.7 & 2.9 & 2.9 & 1.2 & 1.8 & 6.1 & 3.6 & 2.8 & 2.4 \\
\hline 5 & 1.7 & 3.7 & 4.9 & 1.8 & 1.3 & 3.1 & 2.1 & 2.3 & 1.6 \\
\hline 6 & 1.4 & 2.2 & 2.2 & 1.5 & 1.7 & 2.4 & 1.6 & 1.9 & 1.6 \\
\hline 7 & 1.3 & 1.8 & 2.9 & 1.3 & 1.9 & 1.8 & 1.7 & 1.3 & 1.9 \\
\hline 8 & 2.3 & 2.3 & 2.1 & 2.3 & 1.9 & 1.4 & 1.1 & 3.0 & 2.5 \\
\hline 9 & 2.1 & 2.0 & 2.0 & 1.9 & 1.8 & 2.3 & 1.2 & 1.8 & 2.7 \\
\hline 10 & 3.8 & 2.2 & 1.7 & 5.9 & 1.6 & 2.1 & 1.4 & 1.0 & 3.2 \\
\hline 11 & 3.9 & 2.6 & 1.7 & 2.0 & 1.8 & 7.6 & 1.6 & 4.3 & 1.3 \\
\hline 12 & 2.0 & 1.4 & 6.3 & 1.8 & 3.2 & 5.4 & 1.8 & 1.6 & 8.4 \\
\hline 13 & 1.4 & 1.0 & 2.3 & 1.4 & 1.6 & 5.5 & 1.4 & 1.4 & 3.3 \\
\hline 14 & 1.4 & 1.6 & 2.5 & 1.1 & 2.2 & 6.3 & 1.2 & 1.8 & 1.8 \\
\hline 15 & 1.2 & 1.2 & 2.9 & 1.3 & 3.6 & 1.8 & 1.5 & 1.8 & 2.2 \\
\hline 16 & 1.3 & 1.7 & 4.0 & 1.4 & 2.7 & 2.2 & 1.8 & 1.3 & 5.6 \\
\hline 17 & 5.0 & 1.2 & 3.1 & 1.2 & 1.8 & 1.8 & 1.8 & 1.8 & 4.0 \\
\hline 18 & 1.5 & 2.0 & 3.7 & 1.4 & 1.2 & 2.5 & 1.5 & 1.4 & 4.7 \\
\hline 19 & 4.1 & 1.9 & 6.7 & 1.3 & 1.4 & 1.2 & 1.7 & 1.1 & 4.1 \\
\hline 20 & 2.1 & 1.9 & 2.1 & 1.6 & 1.1 & 2.0 & 1.6 & 1.3 & 2.3 \\
\hline 21 & 2.0 & 2.0 & 4.0 & 1.1 & 1.1 & 2.4 & 4.7 & 1.4 & 1.8 \\
\hline 22 & 4.3 & 1.7 & 3.1 & 1.3 & 1.8 & 1.9 & 1.9 & 1.4 & 4.1 \\
\hline 23 & 1.4 & 1.1 & 2.2 & 1.1 & 1.5 & 1.5 & 3.4 & 1.0 & 1.1 \\
\hline 24 & 1.0 & 1.1 & 1.8 & 1.0 & 1.0 & 1.8 & 2.6 & 1.0 & 3.1 \\
\hline Total & 51.9 & 45.9 & 72.3 & 38.1 & 45.9 & 69.9 & 46.5 & 42.4 & 73.0 \\
\hline Mean & 2.2 & 1.9 & 3.0 & 1.6 & 1.9 & 2.9 & 1.9 & 1.8 & 3.0 \\
\hline$\sigma$ & 1.1 & 0.6 & 1.4 & 1.0 & 0.8 & 1.8 & 0.8 & 0.8 & 1.6 \\
\hline & & & & & & & & & \\
\hline 19
\end{tabular}


Table F.12 Operator IV - Tests 5, 6, and 7

\begin{tabular}{|c|r|r|r|r|r|r|r|r|r|}
\cline { 2 - 11 } \multicolumn{1}{c|}{} & \multicolumn{3}{|c|}{ Test 5 } & \multicolumn{3}{c|}{ Test 6 } & \multicolumn{3}{c|}{ Test 7} \\
\cline { 2 - 11 } \multicolumn{1}{c|}{} & L & M & \multicolumn{1}{c|}{ Q } & \multicolumn{1}{c|}{ A } & \multicolumn{1}{c|}{ D } & \multicolumn{1}{c|}{ G } & J & N & \multicolumn{1}{c|}{ R } \\
\hline 1 & 1.1 & 1.7 & 1.9 & 1.9 & 1.5 & 2.6 & 1.2 & 3.3 & 1.2 \\
\hline 2 & 1.2 & 1.1 & 1.1 & 2.9 & 1.7 & 2.4 & 1.4 & 3.5 & 3.9 \\
\hline 3 & 1.0 & 1.8 & 1.9 & 2.0 & 1.4 & 2.1 & 2.3 & 3.0 & 7.5 \\
\hline 4 & 1.4 & 1.4 & 1.7 & 1.3 & 6.5 & 2.9 & 1.7 & 2.6 & 2.8 \\
\hline 5 & 1.3 & 1.1 & 1.9 & 1.2 & 3.1 & 1.6 & 1.3 & 2.3 & 1.8 \\
\hline 6 & 1.2 & 1.1 & 1.7 & 1.3 & 1.2 & 1.6 & 1.3 & 2.4 & 4.4 \\
\hline 7 & 1.9 & 1.4 & 6.1 & 1.6 & 1.4 & 1.9 & 1.4 & 2.6 & 2.7 \\
\hline 8 & 1.3 & 1.1 & 1.7 & 1.3 & 1.4 & 2.8 & 1.4 & 2.6 & 3.0 \\
\hline 9 & 1.2 & 1.3 & 1.6 & 2.2 & 2.1 & 2.7 & 1.5 & 1.4 & 3.0 \\
\hline 10 & 1.8 & 1.5 & 4.1 & 2.0 & 2.5 & 4.8 & 1.2 & 2.0 & 2.0 \\
\hline 11 & 1.4 & 1.9 & 1.5 & 1.0 & 1.9 & 2.2 & 2.1 & 1.6 & 4.2 \\
\hline 12 & 1.4 & 1.8 & 1.6 & 1.1 & 2.0 & 3.4 & 2.6 & 2.0 & 4.3 \\
\hline 13 & 3.9 & 1.4 & 1.9 & 1.0 & 2.7 & 4.0 & 2.8 & 1.2 & 3.8 \\
\hline 14 & 1.1 & 2.4 & 2.1 & 1.2 & 2.4 & 8.1 & 2.6 & 2.0 & 10.0 \\
\hline 15 & 1.0 & 2.0 & 11.4 & 1.1 & 1.4 & 3.1 & 2.0 & 1.4 & 2.6 \\
\hline 16 & 1.0 & 1.6 & 2.1 & 1.0 & 6.2 & 2.2 & 3.7 & 2.4 & 2.7 \\
\hline 17 & 5.2 & 1.5 & 3.9 & 1.1 & 2.6 & 4.8 & 2.5 & 1.7 & 2.1 \\
\hline 18 & 1.4 & 3.0 & 5.1 & 0.9 & 2.4 & 4.0 & 1.3 & 1.8 & 2.4 \\
\hline 19 & 1.3 & 1.6 & 2.3 & 1.1 & 2.9 & 5.0 & 1.3 & 2.0 & 1.2 \\
\hline 20 & 1.3 & 1.7 & 3.1 & 2.0 & 2.2 & 1.7 & 2.5 & 1.8 & 2.8 \\
\hline 21 & 1.5 & 0.9 & 3.3 & 1.7 & 2.1 & 1.8 & 3.2 & 2.3 & 3.1 \\
\hline 22 & 1.3 & 1.0 & 2.5 & 1.3 & 1.7 & 2.4 & 1.8 & 1.3 & 2.1 \\
\hline 23 & 1.1 & 1.0 & 1.4 & 1.0 & 1.2 & 1.5 & 1.5 & 2.0 & 2.8 \\
\hline 24 & 1.0 & 1.0 & 2.4 & 5.0 & 1.1 & 3.1 & 2.7 & 1.4 & 1.9 \\
\hline Total & 37.5 & 36.5 & 68.3 & 38.5 & 55.7 & 72.9 & 47.4 & 50.7 & 78.2 \\
\hline Mean & 1.6 & 1.5 & 2.8 & 1.6 & 2.3 & 3.0 & 2.0 & 2.1 & 3.3 \\
\hline$\sigma$ & 1.0 & 0.5 & 2.2 & 0.9 & 1.3 & 1.5 & 0.7 & 0.6 & 1.9 \\
\hline
\end{tabular}




\section{APPENDIX G}

\section{Operator Learning Curves}

Estimation of the operators' learning curves involved four assumptions.

- The learning curves had positive slope at all times.

- The learning curves were smooth functions.

- The learning curves were exponential functions.

- For a specified field of view, operator learning was reflected primarily in the standard deviation of the normalized course times (a smoothness measure).

Each operator drove 21 courses with a $60^{\circ}$ field of view, three for each of the seven tests. The procedure below was applied to the normalized data for each operator to estimate his $60^{\circ} \mathrm{FOV}$ learning curve.

- The standard deviations were entered into a $3 \times 7$ matrix. Each row represented a specific window width $\beta$ as discussed in section 5.2 (row $1=15^{\circ}$, row 2 $=30^{\circ}$, and row $3=60^{\circ}$ ). Each column corresponded to a given test (column $1=$ test 1 , column $2=$ test 2 , etc. $)$.

- The standard deviations for the three window widths for each test were summed and entered into a row vector of seven elements. Each column continued to represent a given test as above. This step yielded the summed standard deviations (SSD).

- The row vector elements were plotted with summed standard deviations on the vertical axis versus test number on the horizontal axis.

- An exponential curve using the method of least squares was fitted to the plot by linearizing the exponential form through taking logarithms [G-1]. This 
defined the operator's $60^{\circ}$ FOV learning curve.

Figures G.1 through G.4 show the learning curves (graphed as lines) versus the test number. 


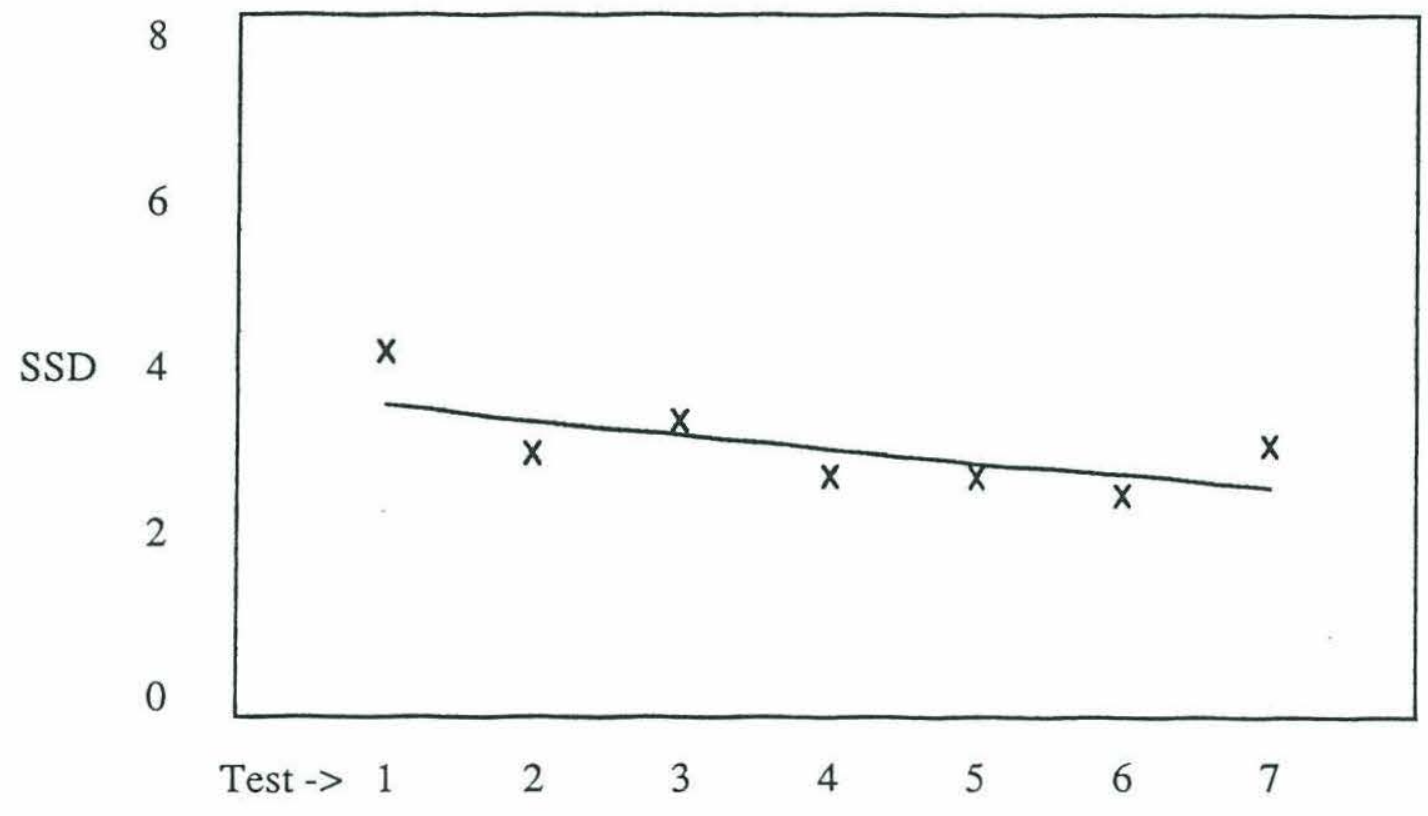

Figure G.1 Operator I $60^{\circ}$ FOV learning curve

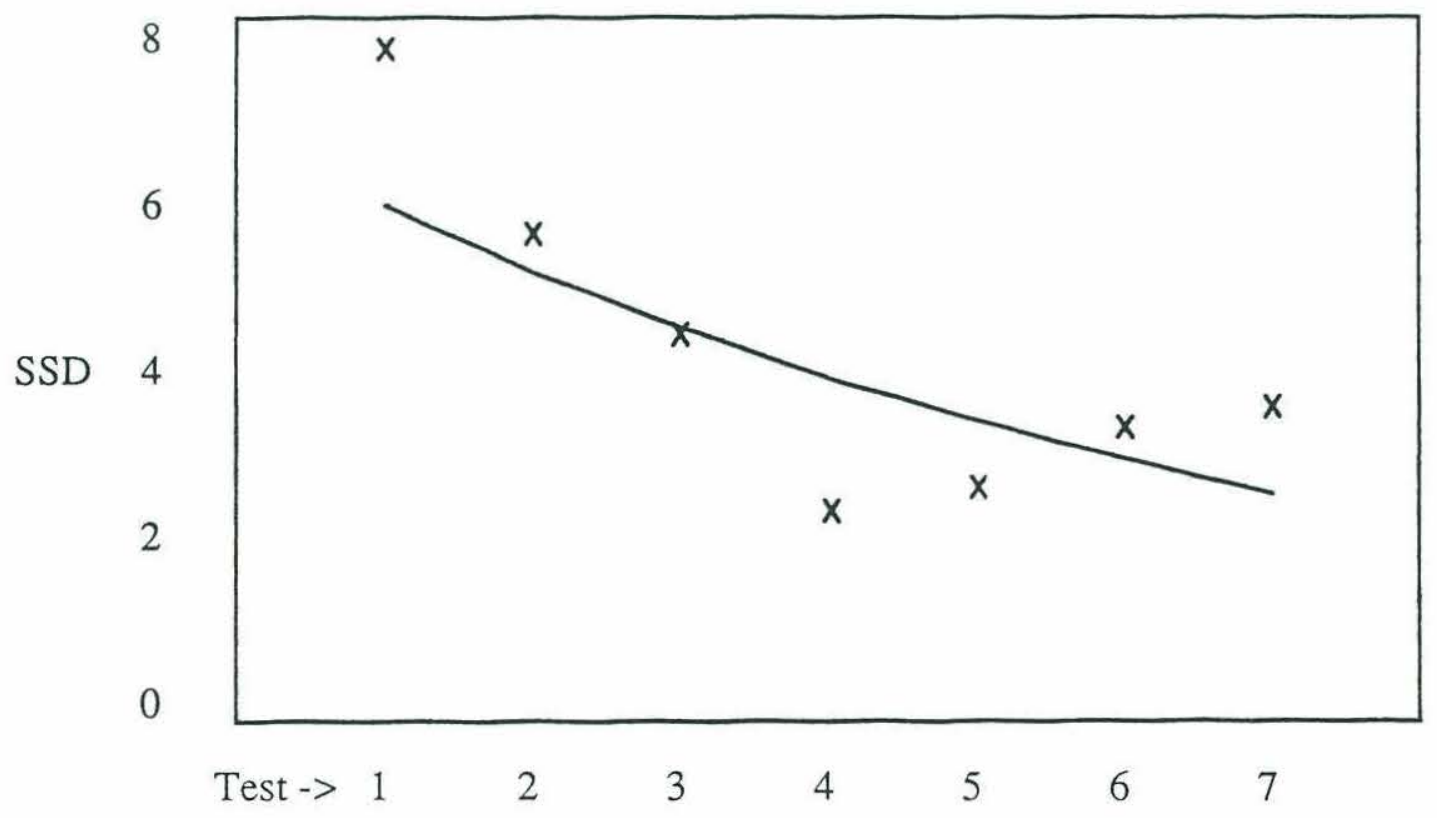

Figure G.2 Operator II $60^{\circ}$ FOV learning curve 


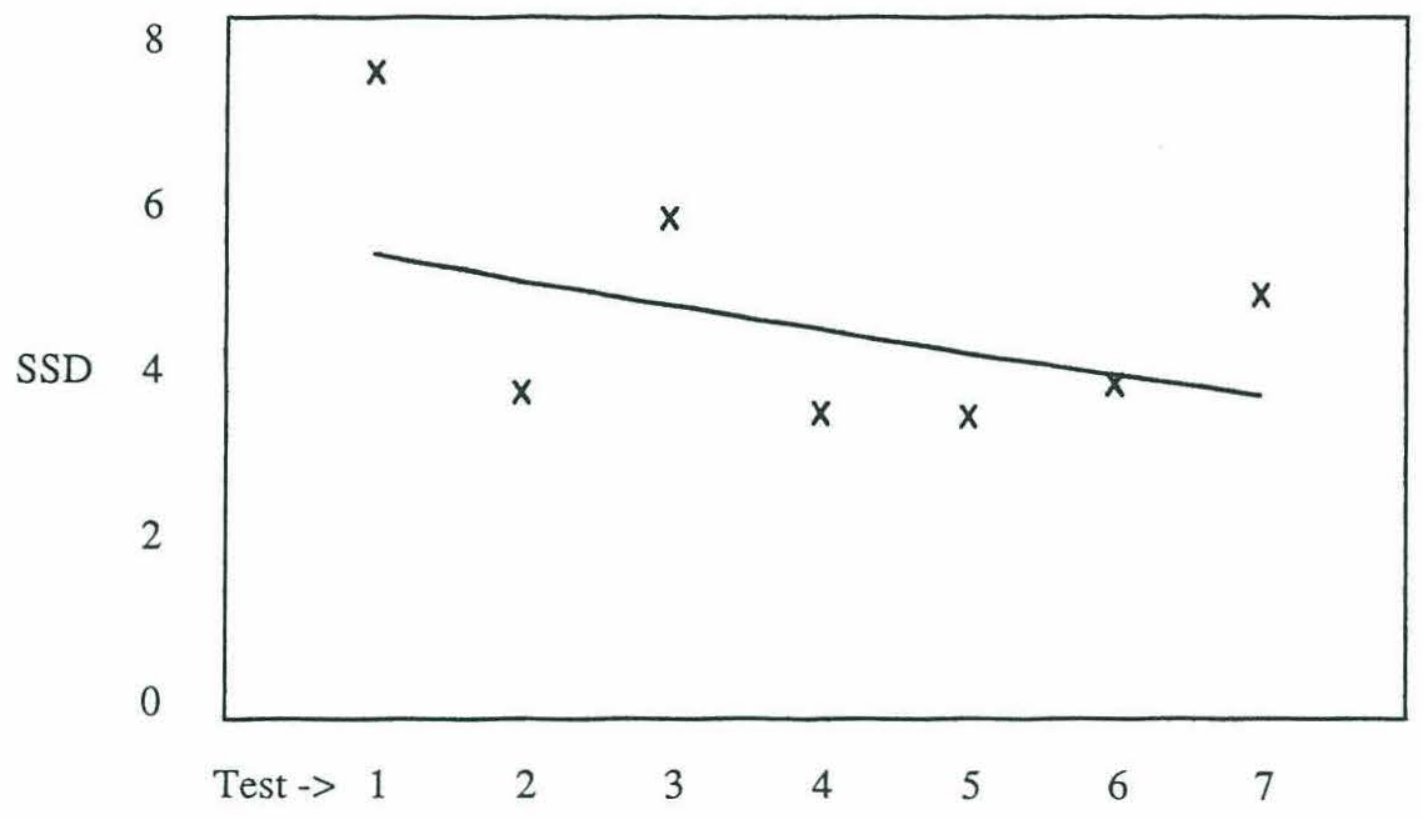

Figure G.3 Operator III $60^{\circ}$ FOV learning curve

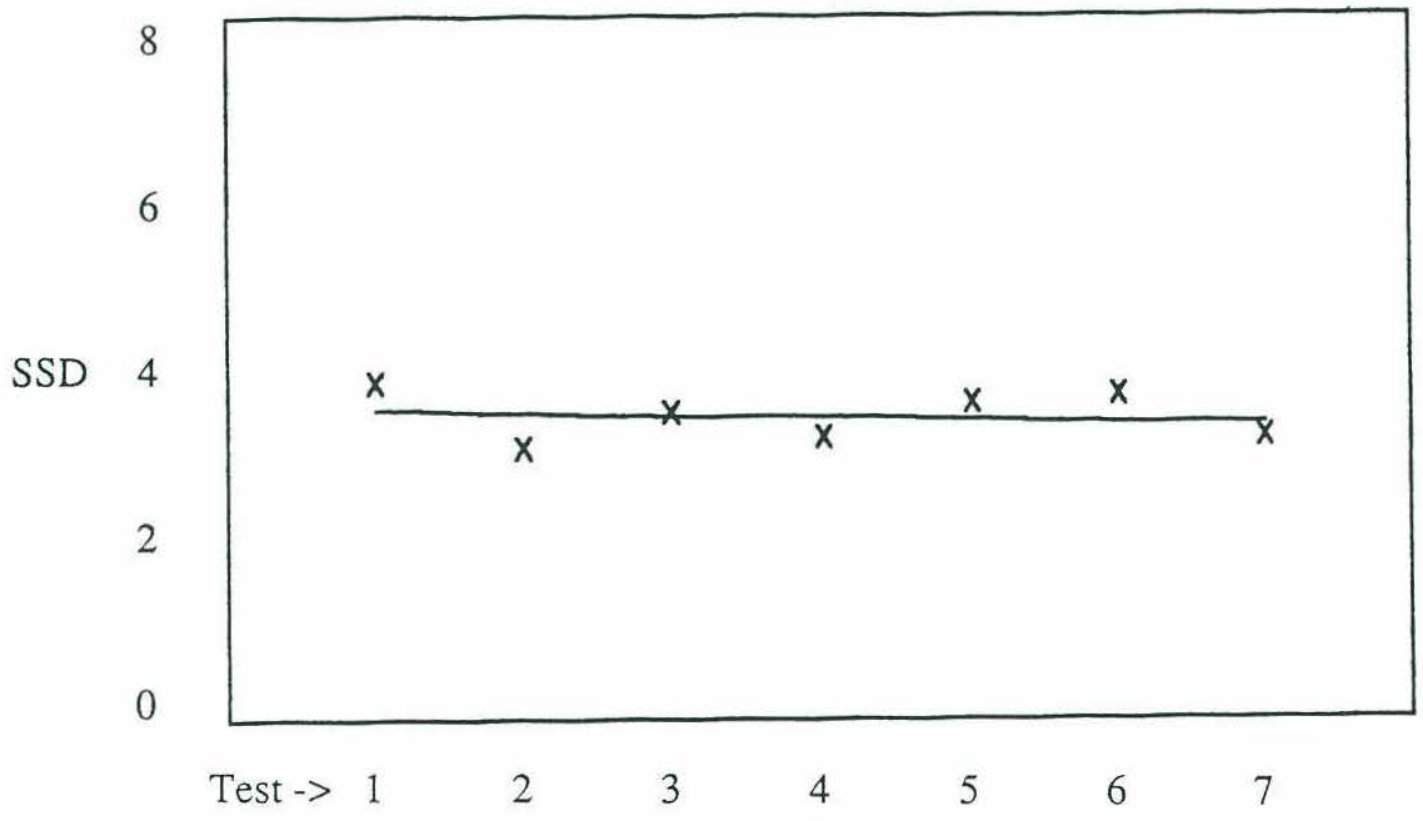

Figure G.4 Operator IV $60^{\circ}$ FOV learning curve 


\section{APPENDIX $\mathbf{H}$}

\section{References}

B-1 T. Berk, L. Brownston, and A. Kaufman, "A New Color-Naming System for Graphics Languages," IEEE Computer Graphics and Applications, Vol. 2, No. 3, (May 1982).

C-1 I. Carlbom and J. Paciorek, "Planar Geometric Projections and Viewing Transformations," ACM Computing Surveys, Vol. 10, No. 4, (December 1978).

E-1 A. Emmett, "In Search of the Miracle Hologram," Computer Graphics World, Vol. 14, No. 2, (February 1991).

F-1 J. D. Foley, A. van Dam, S. K. Feiner, et al., Computer Graphics: Principles and Practice, Addison-Wesley Publishing Company, Inc., Reading, MA, 1990.

G-1 C. F. Gerald and P. O. Wheatley, Applied Numerical Analysis, fourth edition, Addison-Wesley Publishing Company, Inc., Reading, MA, 1990.

K-1 K. L. Kelly and D. B. Judd, COLOR: Universal Language and Dictionary of Names, National Bureau of Standards Special Publication 440, U. S. Government Printing Office, Washington, D. C., 1976.

M-1 D. Meister, Behavioral Analysis and Measurement Methods, John Wiley \& Sons, Inc., New York, 1985. 
M-2 F. H. Moffitt and E. M. Mikhail, Photogrammetry, third edition, Harper \& Row, Publishers, Inc., New York, 1980.

S-1 M. S. Sanders and E. J. McCormick, Human Factors in Engineering and Design, sixth edition, McGraw-Hill Book Company, New York, 1987.

S-2 L. D. Sher, personal communication, BBN Laboratories Incorporated, interview February 12, 1991.

S-3 I. E. Sutherland, "The Ultimate Display," in Proceedings of the 1965 IFIP Congress, 2, 1965, 506-508.

S-4 I. E. Sutherland, "A Head-Mounted Three Dimensional Display," in FJCC 1968, Thompson Books, Washington, DC, 757-764.

S-5 W. K. Stewart, "A Non-Deterministic Approach to 3-D Modeling Underwater," in Proceedings of the Symposium on Unmanned Untethered Submersible Technology, Vol. 1, University of New Hampshire, Marine Systems Laboratory, (June 1987).

S-6 W. K. Stewart, "Multisensor Modeling Underwater with Uncertain Information," Ph.D. Thesis, Massachusetts Institute of Technology and Woods Hole Oceanographic Institution, (September 1988).

S-7 W. K. Stewart, "'Visualization Resources and Strategies for Remote Subsea Exploration," Scientific Visualization of Physical Phenomena, Springer-Verlag, 
Hong Kong, 1991, 85-109.

T-1 A. C. Traub, "Stereoscopic Display Using Rapid Varifocal Mirror Oscillations," Applied Optics, Vol. 6, No. 6, (June 1967). 


\section{Biographical Note}

Lieutenant Commander Larry Galvin graduated with distinction from the United States Naval Academy in 1978 with a Bachelor of Science degree in Mathematics. His eleven years of service in the Submarine Force has included tours on USS Skate (SSN-578), USS Whale (SSN-638), and USS Jack (SSN-605). Following completion of graduate work, LCDR Galvin will report to USS Buffalo (SSN-715) as Executive Officer. 